\title{
DES Y1 results: Splitting growth and geometry to test $\Lambda \mathrm{CDM}$
}

J. Muir $\odot,{ }^{1, *}$ E. Baxter $\odot,{ }^{2}$ V. Miranda,${ }^{3}$ C. Doux $\odot,{ }^{4}$ A. Ferte $\odot,{ }^{5}$ C. D. Leonard, ${ }^{6}$ D. Huterer $\odot,{ }^{7}$ B. Jain $\odot,{ }^{4}$ P. Lemos $\odot,{ }^{8}$ M. Raveri $\odot,{ }^{9}$ S. Nadathur $\odot,{ }^{10}$ A. Campos $\odot,{ }^{11,12}$ A. Chen $\odot,{ }^{7}$ S. Dodelson $\odot,{ }^{11}$ J. Elvin-Poole $\odot,{ }^{13,14}$ S. Lee $\odot,{ }^{15}$ L. F. Secco $\odot{ }^{4}$ M. A. Troxel, ${ }^{15}$ N. Weaverdyck $\odot,{ }^{7}$ J. Zuntz,${ }^{16}$ D. Brout $\odot,{ }^{17}$ A. Choi $\odot,{ }^{13}$ M. Crocce, ${ }^{18,19}$ T. M. Davis $\odot,{ }^{20}$ D. Gruen $\odot,{ }^{21,1,22}$ E. Krause $\odot,{ }^{3}$ C. Lidman $\odot,{ }^{23,24}$ N. MacCrann, ${ }^{13,14}$ A. Möller $\odot,{ }^{25}$ J. Prat $\odot,{ }^{26}$ A. J. Ross $\odot,{ }^{13}$ M. Sako $\odot,{ }^{4}$ S. Samuroff $\odot,{ }^{11}$ C. Sánchez $\odot,{ }^{4}$ D. Scolnic $\odot,{ }^{15}$ B. Zhang $\odot,{ }^{24}$ T. M. C. Abbott $\odot,{ }^{27}$ M. Aguena $\odot,{ }^{28,29}$ S. Allam,${ }^{30}$ J. Annis $\odot,{ }^{30}$ S. Avila $\odot,{ }^{31}$ D. Bacon $\odot,{ }^{10}$ E. Bertin $\odot,{ }^{32,33}$ S. Bhargava, ${ }^{34}$ S. L. Bridle $\odot,{ }^{35}$ D. Brooks $\odot,{ }^{8}$ D. L. Burke $\odot,{ }^{1,22}$ A. Carnero Rosell, ${ }^{36,37}$ M. Carrasco Kind $\odot,{ }^{38,39}$ J. Carretero $\odot,{ }^{40}$ R. Cawthon $\odot,{ }^{41}$ M. Costanzi $\odot,{ }^{42,43}$ L. N. da Costa $\odot,{ }^{29,44}$ M. E. S. Pereira $\odot,{ }^{7}$ S. Desai $\odot,{ }^{45}$ H. T. Diehl, ${ }^{30}$ J. P. Dietrich $\odot,{ }^{46}$ P. Doel $\odot,{ }^{8}$ J. Estrada, ${ }^{30}$ S. Everett $\odot,{ }^{47}$ A. E. Evrard $\odot{ }^{48,7}$ I. Ferrero $\odot{ }^{49}$ B. Flaugher, ${ }^{30}$ J. Frieman $\odot,{ }^{30,9}$ J. García-Bellido $\odot{ }^{31}$ T. Giannantonio $\odot,{ }^{50,51}$ R. A. Gruendl $\odot,{ }^{38,39}$

J. Gschwend $\odot,{ }^{29,44}$ G. Gutierrez, ${ }^{30}$ S. R. Hinton, ${ }^{20}$ D. L. Hollowood $\odot,{ }^{47}$ K. Honscheid, ${ }^{13,14}$ B. Hoyle, ${ }^{46,52,53}$ D. J. James, ${ }^{54}$ T. Jeltema $\odot,{ }^{47}$ K. Kuehn $\odot,{ }^{5,56}$ N. Kuropatkin $\odot{ }^{30}$ O. Lahav, ${ }^{8}$ M. Lima $\odot,{ }^{28,29}$ M. A. G. Maia $\odot,{ }^{29,44}$

F. Menanteau $\odot,{ }^{38,39}$ R. Miquel $\odot,{ }^{57,40}$ R. Morgan $\odot,{ }^{41}$ J. Myles $\odot,{ }^{21}$ A. Palmese $\odot,{ }^{30,9}$ F. Paz-Chinchón $\odot,{ }^{50,39}$

A. A. Plazas $\odot,{ }^{58}$ A. K. Romer, ${ }^{34}$ A. Roodman $\odot,{ }^{1,22}$ E. Sanchez $\odot,{ }^{59}$ V. Scarpine, ${ }^{30}$ S. Serrano $\odot,{ }^{18,19}$ I. Sevilla-Noarbe $\odot,{ }^{59}$ M. Smith $\odot,{ }^{60}$ E. Suchyta $\odot,{ }^{61}$ M. E. C. Swanson, ${ }_{52}^{39}$ G. Tarle $\odot,{ }_{52,53}^{7}$ D. Thomas $\odot,{ }^{10}$ C. To $\odot,{ }^{21,1,22}$ D. L. Tucker, ${ }^{30}$ T. N. Varga $\odot,{ }^{52,53}$ J. Weller $\odot,{ }^{52,53}$ and R. D. Wilkinson ${ }^{34}$

(DES Collaboration)

\author{
${ }^{1}$ Kavli Institute for Particle Astrophysics \& Cosmology, Stanford University, \\ P.O. Box 2450, Stanford, California 94305, USA \\ ${ }^{2}$ Institute for Astronomy, University of Hawaii, 2680 Woodlawn Drive, Honolulu, Hawaii 96822, USA \\ ${ }^{3}$ Department of Astronomy/Steward Observatory, University of Arizona, \\ 933 North Cherry Avenue, Tucson, Arizona 85721-0065, USA \\ ${ }^{4}$ Department of Physics and Astronomy, University of Pennsylvania, \\ Philadelphia, Pennsylvania 19104, USA \\ ${ }^{5}$ Jet Propulsion Laboratory, California Institute of Technology, \\ 4800 Oak Grove Drive, Pasadena, California 91109, USA \\ ${ }^{6}$ School of Mathematics, Statistics and Physics, Newcastle University, \\ Newcastle upon Tyne NE1 7RU, United Kingdom \\ ${ }^{7}$ Department of Physics, University of Michigan, Ann Arbor, Michigan 48109, USA \\ ${ }^{8}$ Department of Physics \& Astronomy, University College London, \\ Gower Street, London WC1E 6BT, United Kingdom \\ ${ }^{9}$ Kavli Institute for Cosmological Physics, University of Chicago, Chicago, Illinois 60637, USA \\ ${ }^{10}$ Institute of Cosmology and Gravitation, University of Portsmouth, \\ Portsmouth PO1 3FX, United Kingdom \\ ${ }^{11}$ Department of Physics, Carnegie Mellon University, Pittsburgh, Pennsylvania 15312, USA \\ ${ }^{12}$ Instituto de Física Teórica, Universidade Estadual Paulista, São Paulo 01140, Brazil \\ ${ }^{13}$ Center for Cosmology and Astro-Particle Physics, The Ohio State University, \\ Columbus, Ohio 43210, USA \\ ${ }^{14}$ Department of Physics, The Ohio State University, Columbus, Ohio 43210, USA \\ ${ }^{15}$ Department of Physics, Duke University, Durham, North Carolina 27708, USA \\ ${ }^{16}$ Institute for Astronomy, University of Edinburgh, Edinburgh EH9 3HJ, United Kingdom \\ ${ }^{17}$ Center for Astrophysics, Harvard \& Smithsonian, \\ 60 Garden Street, Cambridge, Massachusetts 02138, USA \\ ${ }^{18}$ Institut d'Estudis Espacials de Catalunya (IEEC), 08034 Barcelona, Spain \\ ${ }^{19}$ Institute of Space Sciences (ICE, CSIC), Campus UAB, Carrer de Can Magrans, s/n, \\ 08193 Barcelona, Spain \\ ${ }^{20}$ School of Mathematics and Physics, University of Queensland, Brisbane, Queensland 4072, Australia \\ ${ }^{21}$ Department of Physics, Stanford University, 382 Via Pueblo Mall, Stanford, California 94305, USA \\ ${ }^{22}$ SLAC National Accelerator Laboratory, Menlo Park, California 94025, USA \\ ${ }^{23}$ Centre for Gravitational Astrophysics, College of Science, The Australian National University, \\ Australian Capital Territory 2601, Canberra, Australia \\ ${ }^{24}$ The Research School of Astronomy and Astrophysics, Australian National University, \\ Australian Capital Territory 2601, Canberra, Australia
}




\author{
${ }^{25}$ Université Clermont Auvergne, CNRS/IN2P3, LPC, F-63000 Clermont-Ferrand, France \\ ${ }^{26}$ Department of Astronomy and Astrophysics, University of Chicago, \\ Chicago, Illinois 60637, USA \\ ${ }^{27}$ Cerro Tololo Inter-American Observatory, NSF's National Optical-Infrared Astronomy Research \\ Laboratory, Casilla 603, La Serena, Chile \\ ${ }^{28}$ Departamento de Física Matemática, Instituto de Física, Universidade de São Paulo, \\ CP 66318, São Paulo, SP 05314-970, Brazil \\ ${ }^{29}$ Laboratório Interinstitucional de e-Astronomia-LIneA, Rua General José Cristino 77, \\ Rio de Janeiro, RJ-20921-400, Brazil \\ ${ }^{30}$ Fermi National Accelerator Laboratory, P.O. Box 500, Batavia, Illinois 60510, USA \\ ${ }^{31}$ Instituto de Fisica Teorica UAM/CSIC, Universidad Autonoma de Madrid, \\ 28049 Madrid, Spain \\ ${ }^{32}$ CNRS, UMR 7095, Institut d'Astrophysique de Paris, F-75014 Paris, France \\ ${ }^{33}$ Sorbonne Universités, UPMC Université Paris 06, UMR 7095, Institut d'Astrophysique de Paris, \\ F-75014 Paris, France \\ ${ }^{34}$ Department of Physics and Astronomy, Pevensey Building, University of Sussex, \\ Brighton BN1 9QH, United Kingdom \\ ${ }^{35}$ Jodrell Bank Center for Astrophysics, School of Physics and Astronomy, University of Manchester, \\ Oxford Road, Manchester M13 9PL, United Kingdom \\ ${ }^{36}$ Instituto de Astrofisica de Canarias, E-38205 La Laguna, Tenerife, Spain \\ ${ }^{37}$ Universidad de La Laguna, Departamento Astrofísica, E-38206 La Laguna, Tenerife, Spain \\ ${ }^{38}$ Department of Astronomy, University of Illinois at Urbana-Champaign, \\ 1002 West Green Street, Urbana, Illinois 61801, USA \\ ${ }^{39}$ National Center for Supercomputing Applications, 1205 West Clark Street, Urbana, Illinois 61801, USA \\ ${ }^{40}$ Institut de Física d'Altes Energies (IFAE), The Barcelona Institute of Science and Technology, \\ Campus UAB, 08193 Bellaterra (Barcelona) Spain \\ ${ }^{41}$ Physics Department, 2320 Chamberlin Hall, University of Wisconsin-Madison, \\ 1150 University Avenue, Madison, Wisconsin 53706-1390, USA \\ ${ }^{42}$ INAF-Osservatorio Astronomico di Trieste, via G. B. Tiepolo 11, I-34143 Trieste, Italy \\ ${ }^{43}$ Institute for Fundamental Physics of the Universe, Via Beirut 2, 34014 Trieste, Italy \\ ${ }^{44}$ Observatório Nacional, Rua General José Cristino 77, Rio de Janeiro, RJ-20921-400, Brazil \\ ${ }^{45}$ Department of Physics, IIT Hyderabad, Kandi, Telangana 502285, India \\ ${ }^{46}$ Faculty of Physics, Ludwig-Maximilians-Universität, Scheinerstrasse 1, 81679 Munich, Germany \\ ${ }^{47}$ Santa Cruz Institute for Particle Physics, Santa Cruz, California 95064, USA \\ ${ }^{48}$ Department of Astronomy, University of Michigan, Ann Arbor, Michigan 48109, USA \\ ${ }^{49}$ Institute of Theoretical Astrophysics, University of Oslo, \\ P.O. Box 1029 Blindern, NO-0315 Oslo, Norway \\ ${ }^{50}$ Institute of Astronomy, University of Cambridge, \\ Madingley Road, Cambridge CB3 OHA, United Kingdom \\ ${ }^{51}$ Kavli Institute for Cosmology, University of Cambridge, \\ Madingley Road, Cambridge CB3 OHA, United Kingdom \\ ${ }^{52}$ Max Planck Institute for Extraterrestrial Physics, Giessenbachstrasse, 85748 Garching, Germany \\ ${ }^{53}$ Universitäts-Sternwarte, Fakultät für Physik, Ludwig-Maximilians Universität München, \\ Scheinerstrasse 1, 81679 München, Germany \\ ${ }^{54}$ Center for Astrophysics I Harvard \& Smithsonian, \\ 60 Garden Street, Cambridge, Massachusetts 02138, USA \\ ${ }^{55}$ Australian Astronomical Optics, Macquarie University, North Ryde, New South Wales 2113, Australia \\ ${ }^{56}$ Lowell Observatory, 1400 Mars Hill Road, Flagstaff, Arizona 86001, USA \\ ${ }^{57}$ Institució Catalana de Recerca i Estudis Avançats, E-08010 Barcelona, Spain \\ ${ }^{58}$ Department of Astrophysical Sciences, Princeton University, \\ Peyton Hall, Princeton, New Jersey 08544, USA \\ ${ }^{59}$ Centro de Investigaciones Energéticas, Medioambientales y Tecnológicas (CIEMAT), \\ Madrid 28040, Spain \\ ${ }^{60}$ School of Physics and Astronomy, University of Southampton, Southampton SO17 1BJ, United Kingdom \\ ${ }^{61}$ Computer Science and Mathematics Division, Oak Ridge National Laboratory, \\ Oak Ridge, Tennessee 37831, USA
}

\footnotetext{
*Corresponding author. jlmuir@stanford.edu
} 
(Received 13 October 2020; accepted 7 December 2020; published 21 January 2021)

We analyze Dark Energy Survey (DES) data to constrain a cosmological model where a subset of parameters-focusing on $\Omega_{m}$ - are split into versions associated with structure growth (e.g., $\Omega_{m}^{\text {grow }}$ ) and expansion history (e.g., $\Omega_{m}^{\mathrm{geo}}$ ). Once the parameters have been specified for the $\Lambda \mathrm{CDM}$ cosmological model, which includes general relativity as a theory of gravity, it uniquely predicts the evolution of both geometry (distances) and the growth of structure over cosmic time. Any inconsistency between measurements of geometry and growth could therefore indicate a breakdown of that model. Our growth-geometry split approach therefore serves both as a (largely) model-independent test for beyond- $\Lambda \mathrm{CDM}$ physics, and as a means to characterize how DES observables provide cosmological information. We analyze the same multiprobe DES data as [Phys. Rev. Lett. 122, 171301 (2019)] : DES Year 1 (Y1) galaxy clustering and weak lensing, which are sensitive to both growth and geometry, as well as Y1 BAO and Y3 supernovae, which probe geometry. We additionally include external geometric information from BOSS DR12 BAO and a compressed Planck 2015 likelihood, and external growth information from BOSS DR12 RSD. We find no significant disagreement with $\Omega_{m}^{\text {grow }}=\Omega_{m}^{\text {geo }}$. When DES and external data are analyzed separately, degeneracies with neutrino mass and intrinsic alignments limit our ability to measure $\Omega_{m}^{\text {grow }}$, but combining DES with external data allows us to constrain both growth and geometric quantities. We also consider a parametrization where we split both $\Omega_{m}$ and $w$, but find that even our most constraining data combination is unable to separately constrain $\Omega_{m}^{\text {grow }}$ and $w^{\text {grow }}$. Relative to $\Lambda \mathrm{CDM}$, splitting growth and geometry weakens bounds on $\sigma_{8}$ but does not alter constraints on $h$.

DOI: 10.1103/PhysRevD.103.023528

\section{INTRODUCTION}

One of the major goals of modern cosmology is to better understand the nature of the dark energy that drives the Universe's accelerating expansion. Though the simplest model for dark energy, a cosmological constant $\Lambda$, is in agreement with nearly all observations to date, there exist a number of viable alternative models which explain the observed acceleration by introducing new fields or by extending general relativity via some form of modified gravity $[1,2]$. Because there is no single most favored theoretical alternative, observational studies of dark energy largely consist of searches for tensions with the predictions of a minimal cosmological model, $\Lambda \mathrm{CDM}$, which consists of a cosmological constant description of dark energy $(\Lambda)$, cold dark matter (CDM), and general relativity as the theory of gravity.

A tension that has attracted significant attention is that between constraints on the amplitude of matter density fluctuations $\sigma_{8}$ made by low redshift measurements-e.g., by the Dark Energy Survey (DES) - and by Planck measurements of the cosmic microwave background (CMB). This comparison is often phrased in terms of $S_{8} \equiv \sigma_{8} \sqrt{\Omega_{m} / 0.3}$, the parameter combination most constrained by weak lensing analyses. Though the DES and Planck results are not in tension according to the statistical metrics used in the original DES Year 1 analysis [3] (note that this is a topic of some discussion [4]), the DES constraints prefer slightly lower $\sigma_{8}$ than those from Planck. This offset is in a direction consistent with other lensing results [5-13] and has been demonstrated to be independent [14] of the much-discussed tension between $\mathrm{CMB}$ and local SNe measurements of the Hubble constant $H_{0}$ [15-17]. In fact, of the numerous theoretical studies focused on alleviating the $H_{0}$ tension, most have found a joint resolution of the $\sigma_{8}$ and $H_{0}$ tensions challenging, as discussed in, e.g., Refs. [18-21]. Independent CMB measurements from ACT and WMAP give $\sigma_{8}$ constraints consistent with those from Planck [22], while constraints based solely on reconstructed Planck CMB lensing maps are consistent with $\sigma_{8}$ constraints from both DES and measurements of CMB temperature and polarization $[23,24]$.

These tensions are interesting because mismatched constraints from low- and high-redshift probes could indicate a need to extend our cosmological model beyond $\Lambda \mathrm{CDM}$. Of course, it is also possible that these offsets could be caused by systematic errors or a statistical fluke. Given this, it is important to examine how different observables contribute to the $\sigma_{8}$ (and $H_{0}$ ) tension, as well as what classes of model extensions have the potential to alleviate them.

With this goal in mind, we perform a consistency test between geometric measurements of expansion history and measurements of the growth of large-scale structure. The motivation for this test is similar to that of the early- vs late-Universe (Planck vs DES) comparison: we want to check for agreement between two classes of cosmological observables that have been split in a physically motivated way. More ambitiously, we can also view this analysis as a search for signs of beyond- $\Lambda \mathrm{CDM}$ physics. The growthgeometry split is motivated in particular by the fact that modified gravity models have been shown to generically 
break the consistency between expansion and structure growth expected in $\Lambda$ CDM [25-29].

Our analysis focuses on data from the Dark Energy Survey. The DES is an imaging survey conducted between 2013 and 2019 which mapped galaxy positions and shapes over a $5000 \mathrm{deg}^{2}$ area and performed a supernova survey in a smaller $27 \mathrm{deg}^{2}$ region. This large survey volume and access to multiple observables make the DES a powerful tool for constraining both expansion history and structure growth. Constraints on cosmological parameters from the first year of DES data (Y1) have been published for the combined analysis of galaxy clustering and weak lensing $[3,30]$, for the baryonic acoustic oscillation (BAO) feature in the galaxy distribution [31], and for galaxy cluster abundance [32]. Additionally, cosmological results have been reported for the first three years (Y3) of supernova data [33], as well as for the combined analysis of $\mathrm{Y} 3 \mathrm{SNe}$ with Y1 galaxy clustering, weak lensing, and BAOs [34]. Analyses of DES Y3 clustering and lensing data are currently underway. The results presented in this paper are based on a multiprobe analysis like that of Ref. [34].

Because weak lensing and large-scale structure probes like those measured by DES mix information from growth and geometry [27,35-39], rather than purely comparing $\Lambda \mathrm{CDM}$ constraints from two datasets, we introduce new parameters to facilitate this comparison. As we explain in more detail in Sec. II, we define separate "growth" and "geometry" versions of a subset of cosmological parameters $\Theta$ : $\Theta^{\text {grow }}$ and $\Theta^{\text {geo }}$. By constraining growth and geometry parameters simultaneously, we can answer questions like

(1) Are DES constraints driven more by growth or geometric information?

(2) Are the data consistent with the predictions of $\Lambda \mathrm{CDM}$ - that is, with $\Theta^{\mathrm{grow}}=\Theta^{\mathrm{geo}}$ ?

(3) Is the DES preference for low $\sigma_{8}$ compared to Planck driven more by its sensitivity to background expansion (geometry) or by its measurement of the evolution of inhomogeneities (growth)?

Our analysis thus serves as both a model-independent search for new physics affecting structure growth and an approach to building a deeper understanding of how DES observables contribute cosmological information.

The closest predecessors to the present work are Refs. [40-42], which introduce similar growth-geometry consistency tests and apply them to data. These analyses have the same general idea and approach as the present analysis, but differ in several important aspects of how they implement the theoretical modeling of observables in their split parametrization. In a similar spirit, Ref. [43] explores growth-geometry consistency without introducing new parameters, using instead dataset comparisons in a search for discordance with $\Lambda \mathrm{CDM}$. These approaches are complemented by other attempts at model-independent tests of dark energy and modified gravity [44,45], including analyses involving metaparameters analogous to our split parametrization [46-48], as well as other parametrizations which allow structure growth to deviate from expectations set by general relativity. These include analyses that have constrained free amplitudes multiplying the growth rate $f \sigma_{8}$ [49], or the "growth index" parameter $\gamma[26,50]$. The commonly studied $\Sigma-\mu$ model of modified gravity [30,51-54] is also in this category. In fact, the analysis presented below can be viewed as analogous to a $\Sigma$ - $\mu$ study like that in Refs. [55,56], with $\Sigma$ fixed to its GR value, though differences in our physical interpretation of the added parameters changes how we approach analysis choices related to nonlinear scales.

\section{A. Plan of analysis}

Our goal is to test the consistency between DES Year 1 constraints from expansion and those from measurements of the growth of large-scale structure. We will do this using three different combinations of data:

(1) DES data alone (including DES galaxy clustering and weak lensing, BAO, and supernova measurements)—henceforth, "DES-only" or just "DES."

(2) As above, plus external data constraining geometry only from Planck 2015 and BOSS DR12 BAO measurements-henceforth, "DES + Ext-geo."

(3) As above, plus external growth information from BOSS DR12 RSD measurements-henceforth, "DES + Ext-all."

Our main results will come from the combination of all of these datasets, but we will use the DES-only and DES + Ext-geo subsets to aid our interpretation of how different probes contribute information. ${ }^{1}$

The motivation for this growth-geometry split parametrization is to study the mechanism behind late-time acceleration, so we focus on splitting parameters associated with dark energy properties. Primarily, we will focus on the case where we split the matter density parameter $\Omega_{m}$ in flat $\Lambda \mathrm{CDM}$ - that is,

$$
\Omega_{m} \rightarrow\left\{\Omega_{m}^{\text {geo }}, \Omega_{m}^{\text {grow }}\right\} \quad\left[\text { Split } \Omega_{m}\right] .
$$

As we discuss in more detail below, with some caveats, this split essentially means that $\Omega_{m}^{\text {geo }}$ controls quantities like comoving and angular distances, while $\Omega_{m}^{\text {grow }}$ controls quantities like the growth factor. Because we impose the relation $\Omega_{m}+\Omega_{\Lambda}=1$, this means we also split $\Omega_{\Lambda}$, and $\Omega_{m}^{\text {grow }} \neq \Omega_{m}^{\text {geo }}$ necessarily implies $\Omega_{\Lambda}^{\text {growth }} \neq \Omega_{\Lambda}^{\text {geo }}$.

We will additionally show limited $w \mathrm{CDM}$ results where we split both $\Omega_{m}$ and the dark energy equation of state, $w$-that is,

$$
\left\{\Omega_{m}, w\right\} \rightarrow\left\{\Omega_{m}^{\text {geo }}, \Omega_{m}^{\text {grow }} w^{\text {geo }}, w^{\text {grow }}\right\} \quad\left[\text { Split } \Omega_{m}, w\right] .
$$

\footnotetext{
${ }^{\mathrm{I}}$ We do not include constraints from Planck 2018 [57], eBOSS DR14 [58-60], or eBOSS DR16 [61], because those likelihoods were not available when we set up this analysis. At the end of this paper, in Sec. VII E, we will briefly discuss how updating to use those datasets might influence our results.
} 
TABLE I. Modeling summary.

\begin{tabular}{|c|c|c|c|c|}
\hline Observable & Modeling ingredient & Described in & Geometry & Growth \\
\hline Galaxy clustering and lensing & $\begin{array}{l}P(k) \text { shape at } z_{i} \\
P(k) \text { evolution since } z_{i} \\
\text { Projection to } 2 \mathrm{PCF} \\
\text { Intrinsic alignments }\end{array}$ & $\begin{array}{l}\text { Sec. II A } \\
\text { Sec. II A } \\
\text { Sec. II B } \\
\text { Sec. II B }\end{array}$ & $\begin{array}{l}\checkmark \\
\checkmark\end{array}$ & $\begin{array}{l}\checkmark \\
\checkmark\end{array}$ \\
\hline $\mathrm{BAO}$ & Distances & Sec. II C & $\checkmark$ & \\
\hline RSD & $\begin{array}{l}f(z) \sigma_{8}(z) / \sigma_{8}(0) \\
\sigma_{8}(z=0)\end{array}$ & $\begin{array}{l}\text { Sec. II D } \\
\text { Sec. II D }\end{array}$ & $\checkmark$ & $\begin{array}{l}\checkmark \\
\checkmark\end{array}$ \\
\hline Supernovae (SN) & Distances & Sec. II E & $\checkmark$ & \\
\hline $\mathrm{CMB}$ & Compressed likelihood & Sec. II F & $\checkmark$ & \\
\hline
\end{tabular}

Similarly to the split $\Omega_{m}$ case, $w^{\text {geo }}$ enters into calculations of comoving distances, while $w^{\text {grow }}$ is used to compute, e.g., the growth factor. We wish to calculate the posteriors for the split parameters given the aforementioned data, and in particular test their consistency (whether $\Theta^{\text {geo }}=\Theta^{\text {grow }}$ ) and identify any tensions.

For the split $\Omega_{m}$ model, we will additionally examine how fitting in the extended growth-geometry split parameter space affects constraints on other parameters, with an eye toward understanding degeneracies between the split parameters and $\sum m_{\nu}, \sigma_{8}, h \equiv H_{0} /\left[100 \mathrm{~km} \mathrm{~s}^{-1} \mathrm{Mpc}^{-1}\right]$, and $A_{\mathrm{IA}}$. This will allow us to build a deeper understanding of how the various datasets we consider provide growth and geometry information. It will also allow us to weigh in on whether nonstandard cosmological structure growth could potentially alleviate tensions between late- and earlyUniverse measurements of $\sigma_{8}$ and $h$.

Unless otherwise noted, we use the same modeling and analysis choices as the DES Year 1 cosmology analyses described in Refs. [30,34,62]. In order to ensure that our results are robust against various modeling choices and priors, we will follow similar blinding and validation procedures to those used in Ref. [30] for the analysis of DES Y1 constraints on beyond-wCDM physics.

The paper is organized as follows: In Sec. II, we describe how we model observables in our growth-geometry split parametrization, and in Sec. III, we introduce the data used to measure those observables. Section IV discusses our analysis procedure, including the steps taken to protect our results from confirmation bias in Sec. IVA, and our approach to quantifying tensions and model comparison in Sec. IV B. We present our main results, which are constraints on the split parameters and their consistency with $\Lambda$ CDM, in Sec. V. Section VI contains additional results characterizing how our growth-geometry split parametrization impacts constraints on other cosmological parameters, including $\sigma_{8}$. We conclude in Sec. VII. We discuss validation tests in detail in Appendixes A-D, and in Appendix $\mathrm{E}$ we show plots of results supplementing those in the main body of the text.

\section{MODELING GROWTH AND GEOMETRY}

We consider several cosmological observables in our analysis: galaxy clustering and lensing, BAO, RSD, supernovae, and the CMB power spectra. We model these observables in a way that explicitly separates information from geometry (i.e., expansion history) and growth. The separation of growth and geometry is immediately clear for some probes-supernovae, for instance, are purely geometric because they directly probe the luminosity distance. For other probes, however, this split is not obvious, or even necessarily unique. Throughout, we endeavor to make physically motivated, self-consistent choices, and will note where past studies of growth and geometry differ. We emphasize that we are not developing a new physical model, but are rather developing a phenomenological split of $\Lambda \mathrm{CDM}$.

Since one of our primary interests is in probing the physics associated with cosmic acceleration, we will use "growth" to describe the evolution of density perturbations in the late Universe. Below, we describe our approach to modeling the observables we consider, and we summarize this information in Table I.

Because structure growth depends primarily on the matter density via $\rho_{m} \propto h^{2} \Omega_{m}$ and we would like to decouple this from expansion-based constraints on $h$, for both our split parametrizations we additionally split the dimensionless Hubble parameter $h \equiv H_{0} /\left(100 \mathrm{~km} \mathrm{~s}^{-1} \mathrm{Mpc}^{-1}\right)$. In practice, we fix $h^{\text {grow }}$ to a fiducial value because it has almost no effect on growth observables: varying $h$ across its full prior range results in fractional changes that are less than a percent for all observables considered. We demonstrate in Appendix D that altering this choice by either not splitting $h$ or marginalizing over $h^{\text {grow }}$ has little impact on our results.

\section{A. Splitting the matter power spectrum}

Several of the observables that we consider depend on the matter power spectrum-namely, galaxy clustering and lensing, RSD, and the CMB power spectrum. The matter power spectrum $P(k, z)$ contains both growth and 
geometric information, so there is not a unique choice for how to compute it within our split parametrization. We choose a simple-to-implement and physically motivated approach. Because we use "growth" to describe the evolution of perturbations in the late Universe, we assume that the early-time shape of the power spectrum is determined by geometric parameters.

More concretely, we construct the split linear power spectrum as a function of wave number $k$ and redshift $z$, $P_{\text {lin }}^{\text {split }}(k, z)$, by combining linear matter power spectra computed separately using geometric or growth parameters:

$$
P_{\text {lin }}^{\text {split }}(k, z) \equiv \frac{P_{\text {lin }}^{\text {geo }}\left(k, z_{i}\right)}{P_{\text {lin }}^{\text {grow }}\left(k, z_{i}\right)} P_{\text {lin }}^{\text {grow }}(k, z),
$$

where $P_{\text {lin }}^{\text {geo }}$ and $P_{\text {lin }}^{\text {grow }}$ are the linear matter power spectra computed in $\Lambda \mathrm{CDM}$ using the geometric and growth parameters, respectively, and $z_{i}$ is an arbitrary redshift choice, to be discussed below. This definition has several desirable properties. First, if the growth and geometric parameters are the same, then it reduces to the standard $\Lambda \mathrm{CDM}$ linear power spectrum. Second, ignoring scale-dependent growth from neutrinos, $P_{\text {lin }}^{\text {grow }}(k, z) / P_{\text {lin }}^{\text {grow }}\left(k, z_{i}\right)=D^{2}(z) / D^{2}\left(z_{i}\right)$, where $D(z)$ is the linear growth factor. Consequently, the growth parameters will effectively control the growth of perturbations from $z_{i}$ to $z$. Third, for $z \ll z_{i}$, this ratio of growth factors approaches 1 , so the early-time matter power spectrum is controlled by the geometric parameters, as desired.

We compute nonlinear corrections to the matter power spectrum using HaloFit [63-65]. HaloFit provides a recipe, calibrated on simulations, for converting the linear matter power spectrum into the nonlinear power spectrum. As arguments to the HaloFit fitting function, we use the mixed linear power spectrum from Eq. (1), and we use the growth versions of the cosmological parameters. By using the growth parameters as arguments to HaloFit, we ensure that nonlinear evolution is controlled by the growth parameters, and that if $\Theta^{\text {grow }}=\Theta^{\text {geo }}$, the resultant power spectrum agrees with that computed in the standard DES analyses of, e.g., Ref. [3]. Although HaloFit has not been explicitly validated for our growth-geometry split model, using it is reasonable, because we are performing a consistency test against $\Lambda \mathrm{CDM}$ rather than implementing a real physical model.

Figure 1 shows how the full nonlinear power spectrum $P(k, z=0)$ is affected by $20 \%$ changes to $\Omega_{m}^{\text {grow }}$ (blue) and $\Omega_{m}^{\text {geo }}$ (red). For comparison, we also show the effect of changes to $\Omega_{m}$ in $\Lambda$ CDM (gray). The main effects of changing $\Omega_{m}^{\text {geo }}$ are a scaling of the normalization of the power spectrum and a change in the wave number where it peaks. This amplitude change occurs because the Poisson equation relates gravitational potential fluctuations $\Phi$ to matter density fluctuations $\delta$ via

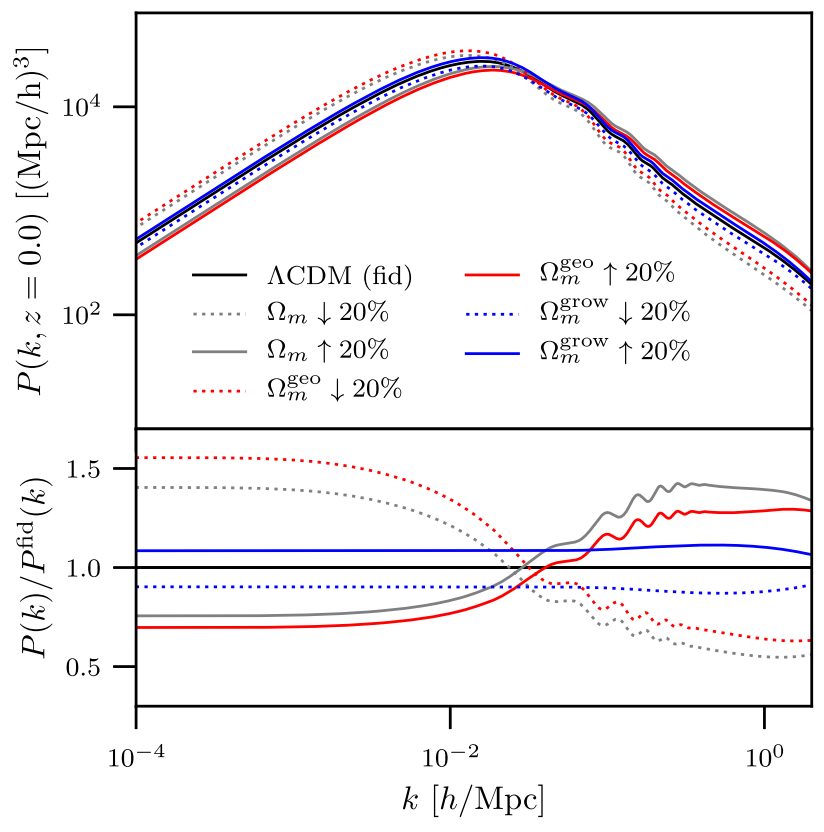

FIG. 1. Dependence of the nonlinear matter power spectrum on $\Omega_{m}^{\text {grow }}$ and $\Omega_{m}^{\text {geo }}$. Gray lines show the impact of changing $\Omega_{m}$ by $\pm 20 \%$ in $\Lambda \mathrm{CDM}$, red lines show changes to $\Omega_{m}^{\mathrm{geo}}$, and blue lines show changes to $\Omega_{m}^{\text {grow }}$. The fiducial model uses $\Omega_{m}^{\text {grow }}=\Omega_{m}^{\text {geo }}=$ 0.295. Solid lines correspond to an increase in the relevant $\Omega_{m}$ parameter to 0.354 , while dotted lines show a decrease to 0.236 .

$$
k^{2} \Phi(k, z)=4 \pi G \rho_{m} \delta=\frac{3}{2} H_{0}^{2} \Omega_{m}(1+z) \delta(k, z) .
$$

Thus, for a fixed primordial potential power spectrum, the matter power spectrum's early-time amplitude is proportional to $\left(\Omega_{m}^{\mathrm{geo}}\right)^{-2}$. The peak of the power spectrum occurs at the wave number corresponding to the horizon scale at matter-radiation equality, $k_{\text {eq }} \propto \Omega_{m} h^{2}$, so increasing $\Omega_{m}^{\text {geo }}$ shifts the peak to higher $k$. Thus, the net effect of increasing $\Omega_{m}^{\mathrm{geo}}$ is a decrease in power at low $k$ and an increase in power at high $k$. Changing $\Omega_{m}^{\text {grow }}$, on the other hand, impacts the late-time growth, leading to a roughly scaleindependent change in the power spectrum. Nonlinear evolution at small scales breaks this scale independence.

We use $z_{i}=3.5$ as our fiducial value for the redshift at which growth parameters start controlling the evolution of the matter power spectrum. This choice is motivated by the fact that $z=3.5$ is before the dark-energy-dominated era and is well beyond the redshift range probed by the DES samples. Raising $z_{i}$ will slightly increase the sensitivity to growth because it means that the growth parameters control a greater portion of the history of structure growth between recombination and the present. However, as long as $z_{i}$ is high enough, this has only a small effect on observables. For the values of $\Omega_{m}^{\text {grow }}$ and $\Omega_{m}^{\text {geo }}$ shown in Fig. 1, we confirm that increasing $z_{i}$ to 5 or 10 results in changes of less than $1 \%$ at all wave numbers of $P_{\text {lin }}^{\text {split }}(k, z=0)$, and 
also at all angular scales of the DES galaxy clustering and weak lensing $2 \mathrm{pt}$ functions. Therefore, the combined constraints of DES and external data are weakly sensitive to the choice of $z_{i}$, as we show in Appendix A.

\section{B. Weak lensing and galaxy clustering}

For a photometric survey like the DES, galaxy and weak lensing correlations are typically measured via angular two-point correlation functions (2PCFs). To make theory predictions for $2 \mathrm{PCF}$, we first compute the angular power spectra. Assuming flat geometry and using the Limber approximation [66,67], the angular power spectrum between the $i$ th redshift bin of tracer $A$ and the $j$ th redshift bin of tracer $B$ is

$$
C_{A B}^{i j}(\ell)=\left.\int d z \frac{H(z)}{c \chi^{2}(z)} W_{A}^{i}(z) W_{B}^{j}(z) P(k, z)\right|_{k=\left(\ell+\frac{1}{2}\right) / \chi(z)} .
$$

Here $\chi$ is the comoving radial distance and $H(z) /\left(c \chi^{2}(z)\right)$ is a volume element that translates three-dimensional density fluctuations into two-dimensional projected number density per redshift. The terms $W_{A}^{i}$ and $W_{B}^{j}$ are window functions relating fluctuations in tracers $A$ and $B$ to the underlying matter density fluctuations whose statistics are described by the power spectrum $P(k, z)$. The window functions for galaxy number density $g$ and weak lensing convergence $\kappa$ are, respectively,

$$
\begin{aligned}
W_{g}^{i}(z, k)= & n_{i}(z) b_{i}(z, k), \\
W_{\kappa}^{i}(z)= & \left(\frac{3 H_{0}^{2} \Omega_{m}}{2 c^{2}}\right)\left(\frac{\chi(z)}{a(z) H(z)}\right) \\
& \times \int_{z}^{\infty} d z^{\prime} n_{i}\left(z^{\prime}\right) \frac{\chi\left(z^{\prime}\right)-\chi(z)}{\chi\left(z^{\prime}\right)} .
\end{aligned}
$$

In these expressions, $n_{i}(z)$ is the normalized redshift distribution of galaxies in sample $i$, while $b_{i}(z, k)$ is their galaxy bias. Following the DES Y1 key paper analysis [3], we will assume a constant linear bias for each sample, denoted with the parameter $b_{i}$.

In our growth-geometry split framework, we compute the power spectrum $P(k, z)$ in Eq. (3) via the procedure described in Sec. II A. We treat all projection operations in Eqs. (3)-(9) as geometric. This choice means that the usual $\sigma_{8}-\Omega_{m}$ weak lensing degeneracy will occur between $\sigma_{8}$ (computed with $P_{\text {lin }}^{\text {split }}$ ) and $\Omega_{m}^{\text {geo }}$, so we define $S_{8} \equiv \sigma_{8} \sqrt{\Omega_{m}^{\mathrm{geo}} / 0.3}$.

We include contributions to galaxy shear correlations from intrinsic alignments between galaxy shapes via a nonlinear alignment model [68], which is the same intrinsic alignment model used in previous DES Y1 analyses [62]. This model adds a term to the shear convergence window function,

$$
W_{\kappa}^{i}(z) \rightarrow W_{\kappa}^{i}(z)-A_{\mathrm{IA}}\left[\left(\frac{1+z}{1+z_{0}}\right)^{\alpha_{I A}} \frac{C_{1} \rho_{m 0}}{D(z)}\right] n_{i}(z) .
$$

Here $A_{\mathrm{IA}}$ and $\alpha_{I A}$ are free parameters which should be marginalized over when performing parameter inference. The normalization $C_{1}=0.0134 / \rho_{\text {crit }}$ is a constant calibrated based on SuperCOSMOS observations [68], $\rho_{m 0}$ is the present-day physical matter density, and $D(z)$ is the linear growth factor. Because intrinsic alignments are caused by cosmic structures, in our split formulation, we compute these quantities using growth parameters.

To obtain real-space angular correlation functions which can be compared to DES measurements, we then transform the angular power spectra of Eq. (3) using Legendre and Hankel transformations. The correlation between galaxy positions in tomographic bins $i$ and $j$ is

$$
w^{i j}(\theta)=\sum_{\ell} \frac{2 \ell+1}{4 \pi} P_{\ell}(\cos \theta) C_{g g}^{i j}(\ell),
$$

where $P_{\ell}(x)$ is the Legendre polynomial of order $\ell$. Shear correlations are computed in the flat-sky approximation as

$$
\begin{aligned}
& \xi_{+}^{i j}(\theta)=\int \frac{d \ell \ell}{2 \pi} J_{0}(\ell \theta) C_{\kappa \kappa}^{i j}(\ell), \\
& \xi_{-}^{i j}(\theta)=\int \frac{d \ell \ell}{2 \pi} J_{4}(\ell \theta) C_{\kappa \kappa}^{i j}(\ell) .
\end{aligned}
$$

In these expressions, $J_{m}(x)$ is a Bessel function of the first kind of order $m$. Finally, the correlation between galaxy positions in bin $i$ and tangential shears in bin $j$-the so-called "galaxy-galaxy lensing" signal-is similarly computed via

$$
\gamma_{t}^{i j}(\theta)=\int \frac{d \ell \ell}{2 \pi} J_{2}(\ell \theta) C_{g k}^{i j}(\ell)
$$

In our analysis, we perform these Fourier transformations using the function tpstat_via_hankel from the NICAEA software [69,70].

Several astrophysical and measurement systematics impact observed correlations for galaxy clustering and weak lensing. In addition to intrinsic alignments, which we addressed above, these include shear calibration and photometric redshift uncertainties. We model these effects following the previously published DES Y1 analyses [62], introducing several nuisance parameters that we marginalize over when performing parameter estimation. This includes a shear calibration parameter $m_{i}$ for each redshift bin $i$ where shear is measured and a photometric redshift bias parameter $\Delta z_{i}$ for each redshift bin $i$. These systematic effects are not cosmology dependent, and so are not impacted by the growth-geometry split. 


\section{Baryon acoustic oscillations}

Baryon acoustic oscillations (BAOs) rely on a characteristic scale imprinted on galaxy clustering which is set by the sound horizon scale at the end of the Compton drag epoch. That characteristic physical sound horizon scale is

$$
r_{d}=\int_{z_{d}}^{\infty} \frac{c_{s}(z)}{H(z)} d z
$$

where $c_{s}$ is the speed of sound, $z_{d}$ is the redshift of the drag epoch, and $H(z)$ is the expansion rate at redshift $z$. Measurements of the BAO feature in galaxy clustering in directions transverse to the line of sight constrain $D_{M}(z) / r_{d}$, where $D_{M}(z)=(1+z) D_{A}(z)$ is the comoving angular diameter distance and $D_{A}(z)$ is the physical angular diameter distance. Line-of-sight measurements, on the other hand, constrain $H(z) r_{d}$. In practice, constraints from $\mathrm{BAO}$ analyses are reported in terms of dimensionless ratios,

$$
\alpha_{\perp}=\frac{D_{M}(z) r_{d}^{\mathrm{fid}}}{D_{M}^{\mathrm{fid}}(z) r_{d}}
$$

and

$$
\alpha_{\|}=\frac{H^{\mathrm{fid}}(z) r_{d}^{\mathrm{fid}}}{H(z) r_{d}}
$$

where the superscript "fid" indicates that the quantity is computed at a fiducial cosmology.

The cosmological information here comes fundamentally from measures of distances via the comparison between the observed scale of the BAO feature and the physical distance $r_{d}$. Given this, in our split parametrization we compute the expressions in Eqs. (11) and (12) using geometric parameters.

\section{Redshift-space distortions}

Redshift-space distortions (RSDs) measure anisotropies in the apparent clustering of matter in redshift space. These distortions are caused by the infall of matter into overdensities, so the RSDs allow us to measure the rate of growth of cosmic structure. RSD constraints are presented in terms of constraints on $f(z) \sigma_{8}(z)$, where $f(z)=$ $d \ln D / d \ln a$ for linear density fluctuation amplitude $D$ and scale factor $a=(1+z)^{-1}$. In our split parametrization, the amplitude $\sigma_{8} \equiv \sigma_{8}(z=0)$ should match the value computed using the mixed power spectrum $P_{\text {lin }}^{\text {split }}$ from Eq. (1), while the time evolution of $\sigma_{8}(z) / \sigma_{8}(0)$ and the growth rate $f(z)$ should be governed by growth parameters.

To achieve this, we proceed as follows: First, following the method used in Planck analyses [71] [see their Eq. (33)], we use our growth parameters to compute

$$
f(z) \sigma_{8}^{\text {grow }}(z)=\frac{\left[\sigma_{8}^{(\delta v)}(z)\right]^{2}}{\sigma_{8}^{\text {grow }}(z)} .
$$

Here the superscript on $\sigma_{8}^{\text {grow }}$ denotes that it was computed within $\Lambda \mathrm{CDM}$ using the growth parameters. The quantity $\sigma_{8}^{(\delta v)}$ is the smoothed density-velocity correlation; it is defined similarly to $\sigma_{8}(z)$, but instead of using the matter power spectrum $P(k, z)$, it is computed by integrating over the linear cross power between the matter density fluctuations $\delta$ and the divergence of the dark matter and baryon (but not neutrino) peculiar velocity fields in Newtonian gauge, $v=-\nabla \mathbf{v}_{N} / H$. Reference [71] motivates this definition by noting that it is close to what is actually being probed by RSD measurements.

In order to make $\sigma_{8}$ consistent with our split matter power spectrum definition from Eq. (1), we multiply Eq. (13) by the $z=0$ ratio of $\sigma_{8}$, computed from $P_{\text {lin }}^{\text {split }}$ and $\sigma_{8}^{\text {grow }}$. The quantity that we use to compare theory with RSD measurements is therefore

$$
f(z) \sigma_{8}(z)=f(z) \sigma_{8}^{\text {grow }}(z) \times \frac{\sigma_{8}(0)}{\sigma_{8}^{\text {grow }}(0)} .
$$

This expression will be consistent with our method of defining the linear power spectrum in Eq. (1) as long as it is evaluated at $z<z_{i}$.

\section{E. Supernovae}

Cosmological information from supernovae comes from measurements of the apparent magnitude of Type Ia supernovae as a function of redshift. Because the absolute luminosity of Type Ia supernovae can be calibrated to serve as standard candles, the observed flux can be used as a distance measure. Even when the value of that absolute luminosity is not calibrated with more local distance measurements, the relationship between observed supernova fluxes and redshifts contains information about how the expansion rate of the Universe has changed over time.

Measurements and model predictions for supernovae are compared in terms of the distance modulus $\mu$, which is related to the luminosity distance $d_{L}$ via

$$
\mu=5 \log \left[d_{L} / 10 \mathrm{pc}\right] .
$$

The observed distance modulus is nominally given by the sum of the apparent magnitude, $m_{B}$, and a term accounting for the combination of the absolute magnitude and the Hubble constant, $M_{0}$.

We follow the approach to computing this used in the DES Y3 supernova analysis [72], also described in Ref. [73], and we use the Cosmosis module associated with the latter paper to perform the calculations. In practice, computing the distance requires a few additional modeling components. These include the width $x_{1}$ and color $\mathcal{C}$ of the 
light curve, which are used to standardize the luminosity of the Type Ia supernovae, as well as a parameter $G_{\text {host }}$ which introduces a step function to account for correlations between supernova luminosity and host galaxy stellar mass $M_{\text {host }}\left(G_{\text {host }}\right.$ is $+1 / 2$ if $M_{\text {host }}>10^{10} M_{\odot},-1 / 2$ if $\left.M_{\text {host }}<10^{10} M_{\odot}\right)$. The final expression for the distance modulus in terms of these parameters is

$$
\mu=m_{B}+\alpha x_{1}-\beta \mathcal{C}+M_{0}+\gamma G_{\text {host }}+\Delta \mu_{\text {bias }} .
$$

Here the calibration parameters $\alpha, \beta$, and $\gamma$ are fit to data using the formalism from Ref. [74], and the selection bias $\Delta \mu_{\text {bias }}$ is calibrated using simulations [75]. The parameter $M_{0}$ is marginalized over during parameter estimation.

The cosmological information in supernova observations comes from distance measurements, so in our split parametrization we compute these quantities using geometric parameters.

\section{F. Cosmic microwave background}

The cosmic microwave background (CMB) anisotropies in temperature and polarization are a rich cosmological observable with information about both growth and geometry. The geometric information primarily consists of the distance to the last scattering surface and the sound horizon size at recombination. Two parameters encapsulate how these distances (and through them, the cosmological parameters) impact the observed $\mathrm{CMB}$ power spectra: the shift parameter [76],

$$
R_{\text {shift }} \equiv \sqrt{\Omega_{m}(100 h)^{2}} D_{M}\left(z_{*}\right) / c,
$$

which describes the location of the first power spectrum peak, and the angular scale of the sound horizon at last scattering $\ell_{A}=\pi / \theta_{*}$,

$$
\ell_{A} \equiv \pi D_{M}\left(z_{*}\right) / r_{s}\left(z_{*}\right) .
$$

Here $z_{*}$ is the redshift of recombination, $D_{M}$ is the comoving angular diameter distance at that redshift, and $r_{s}$ is the comoving sound horizon size. In our split parametrization, we use geometric parameters to compute these quantities.

The CMB is sensitive to late-time structure growth in a few different ways. The ISW effect adds temperature power at low $\ell$ in a way that depends on the linear growth rate, and weak lensing from low- $z$ structure smooths the peaks of the CMB power spectra at high $\ell$. To be self-consistent, the calculation of these effects should use the split power spectrum described in Sec. II A. Adapting the ISW and CMB lensing predictions to our split parametrization would therefore require a modification of the CAMB software [7779] we use to compute power spectra. In order to simplify our analysis, we focus on a subset of measurements from the CMB that are closely tied to geometric observables, independent of late-time growth.

We do this via a compressed likelihood which describes CMB constraints on $R_{\text {shift }}, \ell_{A}, \Omega_{b} h^{2}, n_{s}$, and $A_{\mathrm{s}}$ after marginalizing over all other parameters, including $\sum m_{\nu}$ and $A_{\text {Lens. }}$. This approach is inspired by the fact that the CMB mainly probes expansion history, and thus dark energy, via the geometric information provided by the locations of its acoustic peaks [80], and by the compressed Planck likelihood provided in Ref. [53]; see Sec. III B 2 below for details. In this formulation, we have constructed our CMB observables to be independent of late-time growth, so we compute the model predictions for them with geometric parameters.

\section{G. Modeling summary and comparison to previous work}

Table I summarizes the sensitivity of the probes discussed above to growth and geometry. Briefly, we derive constraints from structure growth from the LSS observables-galaxy clustering, galaxy-galaxy lensing, weak lensing shear, and RSDs-while all probes we consider provide some information about geometry. Constraints from BAOs, supernovae, and the scale of the first peaks of the CMB provide purely geometric information. The LSS observables mix growth and geometry via their dependence on the power spectrum: its shape is set by geometry, while its evolution since $z_{i}=3.5$ is governed by growth parameters. All projections translating from threedimensional matter power to two-dimensional observed correlations are geometry dependent.

We now compare our choices to previous work.

For the $\mathrm{CMB}$, our geometry-growth split choices are motivated by simple implementation and (since our focus is on DES data) the ease of interpretation. In this, we roughly follow the approach in Ref. [41], which also considers a compressed CMB likelihood that is governed purely by geometry. In contrast, Ref. [40] describes CMB fluctuations (and so the sound horizon scale) using growth parameters, then uses geometry parameters in converting physical to angular scales. Reference [42] splits the growth and geometric information in the CMB by multipole, using the TT, TE, and EE power spectra at $\ell>30$ to constrain geometric parameters, and the low- $\ell(<30)$ multipoles, as well as the lensing power spectrum to constrain growth.

For weak lensing, our approach is closest to Ref. [41], with an additional modification in how we model the matter power spectrum, described in Sec. II A. Reference [42] leaves weak lensing out of their analysis, citing the difficulty in separating growth and geometric contributions to those observables. Both Refs. [40,41] compute the matter power spectrum entirely using growth parameters (as opposed to our split parametrization described in Sec. II A), and (like us) they use geometric parameters for projection operations and for the distances used to 
compute the weak lensing kernel. These analyses differ in how they treat the lensing kernel's $\Omega_{M}^{2}$ prefactor [see Eq. (5)]. Reference [40] treats this as a growth quantity, while Ref. [41] considers it part of the lensing window function, and hence a geometric quantity. Our choice, which matches that of Ref. [41], means that $\Omega_{m}^{\text {grow }}$ affects weak lensing observables solely through changes in the matter power spectrum. Though this weakens our ability to constrain $\Omega_{m}^{\text {grow }}$, it has the benefit of making our model more phenomenologically similar to other parametrizations of nonstandard structure growth, making the interpretation of results more easily generalizable.

Our treatment of BAOs and Type Ia supernovae agrees with all previous literature in treating these probes as purely geometrical. Finally, our treatment of the RSDs is subtly different from previous literature on the subject [40-42], which assumed $f \sigma_{8}$ to be determined purely by the growth parameters, Our RSD is mostly determined by the growth of structure, but we allow $\sigma_{8}(z=0)$ to also include geometric parameters via our split parametrization of the matter power spectrum.

\section{DATA AND LIKELIHOODS}

In this section, we describe the data and likelihoods used for our analyses. The datasets and where to find their descriptions are summarized in Table II.

\section{A. DES Year 1 combined data}

In our growth-geometry split analysis of DES data, we perform a combined analysis of DES Y1 galaxy clustering and weak lensing, DES Y1 BAO, and DES Y3 supernova measurements, following a similar methodology to the multiprobe analysis in Ref. [34]. The combination of these measurements will be referred to as "DES" in the reported constraints below. We now describe the constituent measurements.

Galaxy samples used in these measurements were constructed from the DES Y1 Gold catalog [81], which is derived from imaging data taken between August 2013 and February 2014 using the 570 megapixel Dark Energy Camera [82] at CTIO. The data in the catalog cover an area of $1321 \mathrm{deg}^{2}$ in grizY filters and were processed with the DES data management system [83-86].

\section{DES Y1 galaxy clustering and weak lensing}

The DES Y1 combined galaxy clustering and weak lensing analysis, hereafter referred to as $\mathrm{Y} 1-3 \times 2 \mathrm{pt}$, is based on the analysis of three types of angular two-point correlation functions (2PCF): the correlation between the positions of a population of lens galaxies, the correlation between the measured shapes of a population of source galaxies, and the correlation of both lens positions and source shapes. The lens galaxy sample consists of approximately 660000 luminous red galaxies which were found using the redMaGiC algorithm [87] and were selected using luminosity cuts to have relatively small photo- $z$ errors. They are split into five redshift bins with nominal edges at $z=\{0.15,0.3,0.45,0.6,0.75,0.9\}$. Weak lensing shears are measured from the source galaxy sample, which includes $26 \times 10^{6}$ galaxies. These were selected from the Y1 Gold catalog using the Metacalibration [88,89] and NGMIX [90] algorithms, and the BPZ algorithm [91] is used to estimate redshifts. The source galaxies are split into four redshift bins with approximately equal densities, with nominal edges at $z=\{0.2,0.43,0.63,0.9,1.3\}$ [92,93]. For each source bin, a multiplicative shear calibration parameter $m_{i}$ for $i \in\{1,2,3,4\}$ is introduced in order to prevent shear measurement noise and selection effects from biasing cosmological results. Metacalibration provides tight Gaussian priors on these parameters. The redshift distributions for the lens and source galaxies used in the DES Y1 galaxy clustering and weak lensing measurements are shown in Fig. 2. Uncertainties in photometric redshifts are quantified with nine nuisance parameters $\Delta z_{i}^{x}$, which quantify translations of each redshift bin's distribution to $n_{i}^{x}\left(z-\Delta z_{i}^{x}\right)$, where $i$ labels the redshift bin and $x=$ source or lens.

The 2PCF measurements that comprise the $\mathrm{Y} 1-3 \times 2 \mathrm{pt}$ data are presented in Ref. [94] (galaxy-galaxy), Ref. [95] (galaxy-shear), and Ref. [96] (shear-shear). Each 2PCF is measured in 20 logarithmic bins of angular separation from $2.5^{\prime}$ to $250^{\prime}$ using the TreeCorr [97] algorithm. Angular scale cuts are chosen as described in Ref. [62] in order to remove

TABLE II. Table summarizing datasets included and abbreviations for plots.

\begin{tabular}{llccc}
\hline \hline Combination & \multicolumn{1}{c}{ Datasets } & Described in & Geometry & Growth \\
\hline DES & DES Y1 3 $\times$ 2pt (galaxy clustering and WL) & Sec. III A 1 & $\checkmark$ & $\checkmark$ \\
& DES Y1 BAO & Sec. III A 2 & $\checkmark$ & \\
\multirow{5}{*}{ Ext-geo } & DES Y3 + lowZ SNe & Sec. III A 3 & $\checkmark$ \\
& Compressed 2015 Planck likelihood & Sec. III B 2 & $\checkmark$ \\
\multirow{5}{*}{ Ext-all } & BOSS DR12 BAO & Sec. III B 1 & $\checkmark$ \\
& Ext-geo & & $\checkmark$ & \\
& BOSS DR12 RSD & Sec. III C & $\checkmark$ & $\checkmark$ \\
\hline \hline
\end{tabular}




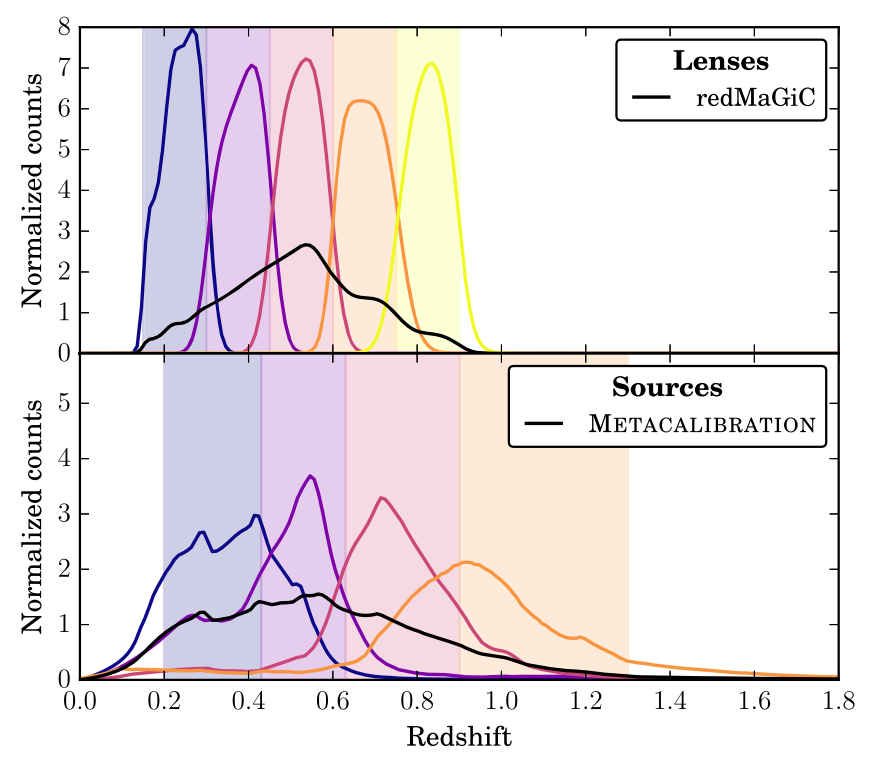

FIG. 2. Redshift distribution of source and lens galaxies used in the DES Y1-3 $\times 2$ pt analysis. The vertical shaded bands represent the nominal range of the redshift bins, while the solid lines show their estimated true redshift distributions, given their photometric-redshift-based selection.

measurements at small angular scales where our model is not expected to accurately describe the impact of nonlinear evolution of the matter power spectrum and baryonic feedback. The resulting DES Y1-3 $\times 2$ pt data vector contains 457 measured $2 \mathrm{PCF}$ values. The likelihood for the $3 \times 2 \mathrm{pt}$ analysis is assumed to be Gaussian in that data vector. The covariance is computed using CosmoLike [98], which employs a halo-model-based calculation of fourpoint functions [99]. References [98,100] present more information about the calculation and validation of the covariance matrix.

\section{DES Y1 BAOS}

The measurement of the signature of baryon acoustic oscillations (BAOs) in DES Y1 data is presented in Ref. [31]. That measurement is summarized as a likelihood of the ratio between the angular diameter distance and the drag scale $D_{A}(z=0.81) / r_{d}$. This result was derived from the analysis of a sample of $1.3 \times 10^{6}$ galaxies from the DES Y1 Gold catalog known as the DES BAO sample. These galaxies in the sample have photometric redshifts between 0.6 and 1.0, and were selected using color and magnitude cuts in order to optimize the high-redshift BAO measurement, as is described in Ref. [101]. An ensemble of 1800 simulations [102] and three different methods for measuring galaxy clustering [103-105] were used to produce the DES BAO likelihood.

The DES BAO sample is measured from the same survey footprint as the samples used in the DES Y1-3 $\times 2 \mathrm{pt}$ analysis, so there will be some correlation between the two measurements. Following Ref. [34], we neglect this correlation when combining the two likelihoods. This can be motivated by the fact that the intersection between the $3 \times 2 \mathrm{pt}$ and $\mathrm{BAO}$ galaxy samples is estimated to be about $14 \%$ of the total BAO sample, and the fact that no significant $\mathrm{BAO}$ signal is measured in the $2 \mathrm{PCF}$ measured for the $3 \times 2 \mathrm{pt}$ analysis.

\section{DES Y3 + low-Z supernovae}

The cosmological analysis of supernova magnitudes from the first three years of DES observations is presented in Ref. [33]. The 207 supernovae used in this analysis were discovered via repeated deep-field observations of a $27 \mathrm{deg}^{2}$ region of the sky taken between August 2013 and February 2016, and are in the redshift range $0.07<z<0.85$. A series of papers describe the search and discovery [86,106,107], calibration [108,109], photometry [110], spectroscopic follow-up [111], simulations [112], selection effects [113], and analysis methodology [72] that went into those results. Following the DES supernova analysis $[33,34]$ (but not the fiducial choices of the multiprobe analysis of Ref. [34]), we additionally include in the supernova sample the so-called low- $z$ subset: 122 supernovae at $z<0.1$ that were measured as part of the Harvard-Smithsonian Center for Astrophysics Surveys [114,115] and the Carnegie Supernova Project [116].

The DES supernova likelihood is a multivariate Gaussian in the difference between the predicted and measured values of the distance modulus $\mu$. The likelihood is implemented in our analysis pipeline using the CosmoSIS Pantheon [73] module, adapted to use the DES measurements instead of the original Pantheon supernova sample.

\section{B. External geometric data}

\section{BOSS DR12 BAOS}

We use BAO information from the constraints presented in the BOSS Data Release 12 [49]. The likelihood provided by BOSS has a default fiducial $r_{d}$ and measurements on $D_{M}(z)$ and $H(z)$ (described in Sec. II C) at the redshifts $z=\{0.38,0.51,0.61\}$. These constraints include measurements of the Hubble parameter $H(z)$ and comoving angular diameter distance $d_{A}(z)$ at redshifts $z=\{0.38,0.51,0.61\}$. Specifically, we use the post-reconstruction BAO-only consensus measurements data file BAO_consensus_ results_dM_Hz.txt and covariance files BAO_ consensüs_covtot_dM_Hz.txt provided on the BOSS results page [117]. No covariance with other data is assumed.

\section{Compressed Planck likelihood}

In order to extract information from Planck data that is independent of our growth parameters, we make our own version of the compressed Planck likelihood presented in 
Ref. [53]. This likelihood is a five-dimensional Gaussian likelihood extracted from a Multinest chain run with the Planck lite 2015 likelihood using the temperature power spectrum (TT) and low- $\ell$ temperature and polarization, with no lensing. We run this chain using the same settings as used for the Planck constraints reported in the DES Y1 papers [3], which includes fixing $w=-1$ and marginalizing over neutrino mass. We also marginalize over the lensing amplitude $A_{\text {Lens }}$ to reduce the possible impact of growth via weak lensing on the temperature power spectrum. From that chain, we extract a $5 \mathrm{D}$ mean and covariance for the parameter vector $\left[R_{\text {shift }}, \ell_{A}, \Omega_{b} h^{2}, n_{s}\right.$, $10^{9} A_{\mathrm{s}}$ ]. The compressed likelihood is then a fivedimensional multivariate Gaussian in those parameters. We confirm that this compressed likelihood is an accurate representation of the Planck constraints in this fivedimensional parameter space-in other words, that the Planck likelihood is approximately Gaussian-by checking that the chain samples for the full Planck likelihood follow a $\chi^{2}$ distribution when evaluated relative to the mean and covariance used in the compressed likelihood.

\section{External growth data (RSD)}

We include an external growth probe using the BOSS DR12 combined results [49]. We use the full-powerspectrum-shape-based consensus measurements data file final_consensus_results_dM_Hz_fsig.tx and the covariance file final_consensus_covtot_dM $\mathrm{Hz}$ fsig.txt provided on the BOSS results page. This includes consensus measurements of $D_{M}(z), H(z)$, and $f(z) \sigma_{8}(z)$ at the same three redshifts $z=\{0.38,0.51$, $0.61\}$ as the BAO-only likelihood. The reported values are the combined results from seven different measurements using different techniques and modeling assumptions, where the covariances between those results have been assessed using mock catalogues [118,119].

As a slight complication, we note that these BOSS results use both the post-reconstruction BAO-only fits described in Sec. III B 1, and those from the full-shape analysis of the pre-reconstruction data. The combination of the post-reconstruction $\mathrm{BAO}$ and pre-reconstruction fullshape fits tightens constraints on $D_{M}(z)$ by around $10 \%$ and those on $H(z)$ by $15 \%-20 \%$. This means that in addition to adding growth information from RSD, our "Ext-all" data combination will also have slightly tighter geometric constraints than "Ext-geo."

\section{ANALYSIS CHOICES AND PROCEDURE}

We use the same parameters and parameter priors as previous DES Y1 analyses [3,30,34]. For our split parameters, we use the same prior as their unsplit counterparts' priors in those previous analyses:

$$
\Omega_{m}, \Omega_{m}^{\text {geo }}, \Omega_{m}^{\text {grow }} \in[0.1,0.9],
$$

$$
w, w^{\mathrm{geo}}, w^{\mathrm{grow}} \in[-2.0,0.33] .
$$

We use the same angular scale cuts for the DES Y1 weak lensing and LSS measurements, leaving 457 data points in the weak lensing and galaxy clustering combined $3 \times 2 \mathrm{pt}$ data vector. The DES BAO likelihood contributes another measurement [of $D_{A}(z=0.81) / r_{d}$ ], and the DES SNe likelihood is based on measurements of 329 supernovae (207 from DES, 122 from the low- $z$ sample). This means that our DES-only analysis is based on a total of 787 data points. The DES + Ext-geo analysis therefore has 798 data points (787 from DES, 5 from compressed Planck, 6 from BOSS BAO), and the DES + Ext-all analysis has 801 (same as DES + Ext-geo, plus 3 BOSS RSD measurements).

Calculations were done in the CosmoSIS [120] software package [121], using the same pipeline as the Y1KP, modulo changes to implement the growth-geometry split. For validation tests, chains were run with the MultiNest sampler [122-124], with low-resolution fast settings of 250 live points, efficiency 0.3 , and tolerance 0.01 . For fits to data where we need both posteriors and Bayesian evidence, we use Polychord [125] with 250 live points, 30 repeats, and tolerance of 0.01 . Summary statistics and contour plots from chains are done using the GetDist [126] software with a smoothing kernel of 0.5 .

As noted in Sec. I A, our main results will be products of parameter estimation and model comparison evaluated for

(1) Split $\Omega_{m}$ constrained with DES + Ext-geo, and

(2) Split $\Omega_{m}$ constrained with DES + Ext-all.

This choice was based on simulated analyses performed before running parameter estimation on real data. In these analyses, we computed model predictions for observables at a fiducial cosmology, then analyzed those predictions as if they were measurements. By studying the relationship between the resulting posteriors and the input parameter values we identified which model-data combinations are constraining enough so that parameter estimates are unbiased by parameter-space projection effects. This is described in more detail in Appendix B. For the DES + Ext-geo and DES + Ext-all constraints on split $\Omega_{m}$, we confirm that the input parameter values are contained within the $68 \%$ confidence intervals of the synthetic-data versions of all marginalized posteriors plotted in this paper.

We consider two additional sets of constraints:

(1) Split $\Omega_{m}$ constrained by DES only.

(2) Split $\Omega_{m}$ and $w$ constrained by DES + Ext-all.

Our simulated analyses reveal that the one-dimensional marginalized posteriors are impacted by significant projection effects. Given this, for these cases we do not report numerical parameter estimates or error bars, but we will still report model comparison statistics (to be discussed in Sec. IV B) and show their two-dimensional 
confidence regions on plots. We do not consider constraints splitting both $\Omega_{m}$ and $w$ for DES-only and DES + Ext-geo, because these datasets are less constraining than DES + Ext-all and so are expected to suffer from even more severe projection effects. A more detailed discussion of these projection effects and the parameter degeneracies which cause them can be found in Appendix B.

We follow a procedure similar to that used in Ref. [30] to validate our analysis pipeline. Our goal is to characterize the robustness of our results to reasonable changes to analysis choices, as well as to astrophysical or modeling systematics. The analysis presented in this paper was blinded in the sense that all analysis choices were fixed and we ensured that the pipeline passed a number of predetermined validation tests before we looked at the true cosmological results. The blinding procedure and these tests are described below.

\section{A. Validation}

In planning and executing this study, we took several steps to protect the results against possible experimenter bias, following a procedure similar to the parameter-level blinding strategy used in previous DES Y1 beyond$\Lambda \mathrm{CDM}$ analyses. Key to this were extensive simulated analyses, in which we analyzed model predictions for observables with known input parameters as if they were data. All analysis choices are based on these simulated analyses, including which datasets we focus on and how we report results. Before running our analysis pipeline on real data, we wrote the bulk of this paper's text, including the plan of how the analysis would proceed, and subjected that text to a preliminary stage of DES internal review.

When performing parameter estimation on the real data, we concealed the cosmology results using the following strategies:

(1) We avoided overplotting measured data and theory predictions for observables.

(2) We post-processed all chains so that the mean of the posterior distributions lay on our fiducial cosmology.

(3) We did not look at model comparison measures between our split parametrization and $\Lambda \mathrm{CDM}$.

We maintained these restrictions until we confirmed that the analysis passed several sets of validation tests:

(1) We confirmed that our results cannot be significantly biased by any one of the sample systematics adopted in our validation tests. To do this, we checked that the parameter estimates we reported changed by less than $0.3 \sigma$ when we contaminated synthetic input data with a number of different effects, including nonlinear galaxy bias and a more sophisticated intrinsic alignment model. This test is discussed in Appendix C.
(2) We confirmed that nonoffset $\Lambda \mathrm{CDM}$ chains give results consistent with what Ref. [34] reports. ${ }^{2}$

(3) We studied whether our main results were robust to changes in our analysis pipeline. We found that parameter constraints shift by less than $0.3 \sigma$ when we apply more aggressive cuts to removing nonlinear angular scales, and when we use an alternative set of photometric redshifts.

Our results did change when we replaced the intrinsic alignment model defined in Eq. (6) with one where the amplitude $A_{\mathrm{IA}}$ varies independently in each source redshift bin. Upon further investigation, as detailed in Appendix D, we found that a similar posterior shift manifests in the analysis of synthetic data, so we believe that it is due to a parameter-space projection effect rather than a property of the real DES data. We therefore proceed with the planned analysis despite failing this robustness test, but we add an examination of how intrinsic alignment properties covary with our split parameters to the discussion in Sec. VI.

After passing another stage of internal review, we then finalized the analysis by updating the plots to show nonoffset posteriors, computing tension and model comparison statistics, and writing descriptions of the results. After unblinding, a few changes were made to the analysis: First, we discovered that our real-data results had accidentally been run using Pantheon [73] supernovae, so we reran all chains to include correct DES SN data. While doing this, we additionally made a small change to our compressed Planck likelihood, centering its Gaussian likelihood on the full Planck chain's mean parameter values, rather than on the maximum posterior sample. This choice was motivated by the fact that sampling error in the maximum posterior estimate means that the compressed likelihood is more accurate when centered on the mean. We estimate that centering on the maximum posterior sample was causing the compressed likelihood to be biased by $\sim 0.2 \sigma$ relative to the mean, though we avoided looking at the direction of this bias in parameter space in order to prevent our knowledge of that direction from influencing this choice.

\section{B. Evaluating tensions and model comparison}

There are two senses in which measuring tension is relevant for this analysis. First, we want to check for tension between different datasets in order to determine whether it is sensible to report their combined constraints. Second, we want to test whether our split parametrization results are in tension with $\Lambda \mathrm{CDM}$ (or $w \mathrm{CDM}$ in the case of split $w$ ). For both of these applications, we evaluate tension using

\footnotetext{
${ }^{2}$ The data combinations we use are slightly different from those in Ref. [34], so we simply require that our $\Lambda$ CDM results be reasonably consistent with theirs, rather than identical.
} 
Bayesian suspiciousness $[4,127]$, which we compute using ANESTHETIC $[128,129]$.

Suspiciousness $S$ is a quantity built from the Bayesian evidence ratio $R$ designed to remove dependence of the tension metric on the choice of prior. Let us define $S^{\text {dat }}$ to measure the tension between two datasets $A$ and $B$. The Bayesian evidence ratio between $A$ 's and $B$ 's constraints is

$$
R^{\text {dat }}=\frac{\mathcal{Z}_{A B}}{\mathcal{Z}_{A} \mathcal{Z}_{B}},
$$

where $\mathcal{Z}_{X}=\int d \Theta \mathcal{P}(\Theta \mid X)$ is the Bayesian evidence for dataset $X$ with posterior $\mathcal{P}(\Theta \mid X)$. Generally, values of $R^{\text {dat }}>1$ indicate agreement between $A$ 's and $B$ 's constraints, while $R^{\text {dat }}<1$ indicates tension, though the translation of $R$ values into tension probability depends on the choice of priors $[4,130]$.

The Kullback-Leibler (KL) divergence

$$
\mathcal{D}_{X}=\int d \Theta \mathcal{P}(\Theta \mid X) \log [\mathcal{P}(\Theta \mid X) / \pi(\Theta)]
$$

measures the information gain between the prior and the posterior for constraints based on dataset $X$. The comparison between KL divergences can be used to quantify the probability, given the prior, that constraints from datasets $A$ and $B$ will agree. This information is encapsulated in the information ratio,

$$
\log I^{\text {dat }}=\mathcal{D}_{A}+\mathcal{D}_{B}-\mathcal{D}_{A B},
$$

where $\mathcal{D}_{A B}$ is the KL divergence for the combined analysis of $A$ and $B$. To get Bayesian suspiciousness, we subtract the information ratio from the Bayesian evidence:

$$
\log S^{\mathrm{dat}}=\log R^{\mathrm{dat}}-\log I^{\mathrm{dat}} .
$$

This subtraction makes $S$ insensitive to changes in the choice of priors, as long as those changes do not significantly impact the posterior shape. As with $R$, larger values of $S$ indicate greater agreement between datasets.

To translate this into a more quantitative measure of consistency, we use the fact that the quantity $d-2 \log S$ approximately follows a $\chi_{d}^{2}$ probability distribution, where $d$ is the number of parameters constrained by both datasets. In practice, we determine $d$ by computing the Bayesian model dimensionality $d$ [131], which accounts for the extent to which our posterior is unconstrained (priorbounded) in some parameter-space directions. The model dimensionality for a single set of constraints $X$ is defined as

$$
\frac{\tilde{d}_{X}}{2}=\int d \Theta \mathcal{P}(\Theta \mid X)(\log [\mathcal{P}(\Theta \mid X) / \pi(\Theta)])^{2}-\mathcal{D}_{X}^{2} .
$$

This measures the variance of the gain in information provided by $X$ 's posterior. Though $\tilde{d}$ is generally noninteger, it can be interpreted as the effective number of constrained parameters. To get the value of $d$ that we use for our tension probability calculation, we compute

$$
d \equiv d_{A \cap B}=\tilde{d}_{A}+\tilde{d}_{B}-\tilde{d}_{A B}
$$

Since any parameter constrained by either $A$ or $B$ will also be constrained by their combination, this subtraction will remove the count for any parameter constrained by only one dataset. Thus, $d$ is the effective number of parameters constrained by both datasets. As we noted above, the quantity $d-2 \log S$ approximately follows a $\chi_{d}^{2}$ probability distribution, so we compute the tension probability

$$
p\left(S>S^{\mathrm{dat}}\right)=\int_{d-2 \log S}^{\infty} \chi_{d}^{2}(x) d x,
$$

which quantifies the probability that the datasets $A$ and $B$ would be more discordant than measured. If in our analysis we find $p\left(S>S^{\mathrm{dat}}\right)<5 \%$, we will consider the two datasets to be in tension and will not report parameter constraints from their combination.

We will also use the Bayesian suspiciousness in order to perform model comparison. One can interpret the Bayesian evidence ratio and suspiciousness defined in Eqs. (21)-(24) as a test of the hypothesis that datasets $A$ and $B$ are described by a common set of cosmological parameters as opposed to two independent sets. That can be directly translated into what we would like to determine: Are the data in tension with a single set of parameters describing both growth and geometric observables? We therefore compute

$$
\begin{gathered}
R^{\mathrm{mod}}=\mathcal{Z}_{\Lambda \mathrm{CDM}} / \mathcal{Z}_{\mathrm{mod}}, \\
I^{\mathrm{mod}}=\mathcal{D}_{\Lambda \mathrm{CDM}}-\mathcal{D}_{\mathrm{mod}}, \\
\log S^{\mathrm{mod}}=\log R^{\mathrm{mod}}-\log I^{\mathrm{mod}} .
\end{gathered}
$$

We use the label "mod" to identify these as model comparison statistics. As before, we translate this into a tension probability by computing the Bayesian model dimensionality,

$$
d=d_{\mathrm{mod}}-d_{\Lambda \mathrm{CDM}}
$$

and integrating the expected $\chi_{d}^{2}$ distribution as in Eq. (27). The resulting quantity $p\left(S>S^{\text {mod }}\right)$ measures the probability to exceed the observed tension between growth and geometric observables.

To convert a probability $p$ to an equivalent $N \sigma$ scale, we compute $N$ such that $p$ is the probability that $|x|>N$ for a standard normal distribution, 


$$
\begin{gathered}
p=1-2 \int_{0}^{N}(2 \pi)^{-1 / 2} e^{-x^{2} / 2} d x=\operatorname{erfc}\left(\frac{N}{\sqrt{2}}\right), \\
N=\sqrt{2} \operatorname{erfc}^{-1}(p) .
\end{gathered}
$$

Unless otherwise noted, this double-tail equivalent probability is what will be used to convert probabilities to $N \sigma$. In the specific case we are testing in Sec. V C of whether the difference between the corresponding growth and geometry parameter is greater than zero, a single-tail probability is relevant instead; in that case, we simply multiply $p$ in Eq. (33) by a factor of 2 .

\section{RESULTS: SPLIT PARAMETERS}

Here we present our main results, which are constraints on split parameters and an assessment of whether or not the data are consistent with $\Theta^{\text {grow }}=\Theta^{\text {geo }}$. Section VA reports results for splitting $\Omega_{m}$ (with $w=-1$ ), while results for splitting both $\Omega_{m}$ and $w$ are presented in Sec. VB. We summarize the results in Sec. VD, reporting constraints, tension metrics, and model comparison statistics in Table III.

All datasets considered fulfill the $p\left(S>S^{\text {dat }}\right) \geq 0.05$ prerequisite set in Sec. IV B for reporting combined constraints. Note, however, that while this is strictly true, the $\Lambda$ CDM constraints from DES and Ext-geo, as well as the split $\Omega_{m}$ constraints from DES and Ext-all, are found to have tensions at the $2 \sigma$ threshold. Thus, while we will report these combined results, they should be interpreted with caution.

Note that while one might assume that the $2 \sigma$ tension found between DES and Ext-geo constraints in $\Lambda \mathrm{CDM}$ is related to the familiar Planck-DES $\sigma_{8}$ offset, this is not necessarily the case. This is because the $\sigma_{8}$ tension is generally studied in terms of the constraints from the full CMB power spectrum, while we are only using limited, geometric information from the CMB. When we do examine marginalized $\Lambda \mathrm{CDM}$ posteriors (not shown), we find substantial overlap between the $1 \sigma$ regions of the marginalized DES and Ext-geo constraints on $\sigma_{8}$. Similarly, we find no obvious incompatibility between DES and Ext-geo constraints on any other individual parameter. This $2 \sigma$ tension therefore appears to be related to the higherdimensional properties of the two posteriors.

\section{A. Splitting $\boldsymbol{\Omega}_{\boldsymbol{m}}$}

Figure 3 shows the $68 \%$ and $95 \%$ confidence regions for $\Omega_{m}^{\text {grow }}$ and $\Omega_{m}^{\text {geo }}$ for various data combinations. We study three different comparisons: a comparison between our fiducial DES dataset and a version without the BAOs and $\mathrm{SNe}$ in the top panel; DES plus external geometric (DES + Ext-geo) data in the middle panel; and DES plus external data including RSDs (Ext-all) in the bottom panel. The diagonal gray line corresponds to $\Omega_{m}^{\text {grow }}=\Omega_{m}^{\text {geo }}$.
TABLE III. Summary of results. Parameter errors quoted are $68 \%$ confidence intervals, and $\tilde{d}$ is the Bayesian model dimensionality. The quantity $S$ is Bayesian suspiciousness, with the superscript "dat" denoting an assessment of tension between two

\begin{tabular}{|c|c|c|c|}
\hline$\Lambda \mathrm{CDM}$ & DES & DES + Ext-geo & DES + Ext-all \\
\hline$\Omega_{m}$ & $0.296_{-0.022}^{+0.020}$ & $0.301_{-0.008}^{+0.009}$ & $0.302_{-0.008}^{+0.007}$ \\
\hline$\tilde{d}$ & $14.0 \pm 0.7$ & $18.2 \pm 0.8$ & $15.8 \pm 0.8$ \\
\hline $\log S^{\text {dat }}$ & $\ldots$ & $-1.8 \pm 0.3$ & $-0.8 \pm 0.2$ \\
\hline$p\left(S>S^{\text {dat }}\right)$ & $\cdots$ & $0.04 \pm 0.03$ & $0.25 \pm 0.05$ \\
\hline Equiv. $\sigma$ & $\cdots$ & $2.0 \pm 0.4$ & $1.2 \pm 0.1$ \\
\hline Split $\Omega_{m}$ & DES & DES + Ext-geo & DES + Ext-all \\
\hline$\Omega_{m}^{\text {grow }}-\Omega_{m}^{\text {geo }}$ & $\cdots$ & $0.126_{-0.129}^{+0.228}$ & $0.116_{-0.084}^{+0.100}$ \\
\hline$\Omega_{m}^{\mathrm{geo}}$ & $\cdots$ & $0.300_{-0.008}^{+0.009}$ & $0.304_{-0.008}^{+0.009}$ \\
\hline$\Omega_{m}^{\text {grow }}$ & $\ldots$ & $0.425_{-0.131}^{+0.232}$ & $0.421_{-0.089}^{+0.102}$ \\
\hline$\tilde{d}$ & $15.0 \pm 0.7$ & $18.1 \pm 0.9$ & $19.8 \pm 1.0$ \\
\hline $\log S^{\mathrm{dat}}$ & $\ldots$ & $-1.1 \pm-0.2$ & $-2.0 \pm 0.3$ \\
\hline$p\left(S>S^{\mathrm{dat}}\right)$ & $\cdots$ & $0.13 \pm 0.06$ & $0.05 \pm 0.03$ \\
\hline Equiv. $\sigma$ & $\cdots$ & $1.5 \pm 0.3$ & $2.0 \pm 0.3$ \\
\hline $\log S^{\bmod }$ & $-0.6 \pm 0.2$ & $-1.3 \pm 0.2$ & $-0.4 \pm 0.3$ \\
\hline$p\left(S>S^{\text {mod }}\right)$ & $0.14 \pm 0.08$ & $0.06 \pm 0.05$ & $0.31 \pm 0.07$ \\
\hline Equiv. $\sigma$ & $1.5 \pm 0.4$ & $1.9 \pm 0.4$ & $1.0 \pm 0.1$ \\
\hline$p\left(\Omega_{m}^{\text {grow }}>\Omega_{m}^{\text {geo }}\right)$ & 0.30 & 0.91 & 0.95 \\
\hline 1-tail equiv. $\sigma$ & 0.5 & 1.3 & 1.6 \\
\hline
\end{tabular}
datasets, and "mod" denoting model comparison.

\begin{tabular}{lcc}
\hline \hline$w$ CDM & DES & DES + Ext-all \\
\hline$\Omega_{m}$ & $0.292_{-0.022}^{+0.022}$ & $0.301_{-0.008}^{+0.009}$ \\
$w$ & $-0.911_{-0.076}^{+0.073}$ & $-0.997_{-0.050}^{+0.048}$ \\
$\tilde{d}$ & $16.3 \pm 0.8$ & $17.6 \pm 0.8$ \\
$\log S^{\text {dat }}$ & $\ldots$ & $-1.5 \pm 0.2$ \\
$p\left(S>S^{\text {dat }}\right)$ & $\ldots$ & $0.17 \pm 0.04$ \\
Equiv. $\sigma$ & $\ldots$ & $1.4 \pm 0.1$ \\
\hline \hline
\end{tabular}

\begin{tabular}{lcc}
\hline \hline Split $\Omega_{m}, w$ & oDES & DES + Ext-all \\
\hline$\tilde{d}$ & $15.2 \pm 0.7$ & $18.2 \pm 0.9$ \\
$\log S^{\text {dat }}$ & $\ldots$ & $-2.2 \pm 0.2$ \\
$p\left(S>S^{\text {dat }}\right)$ & $\ldots$ & $0.09 \pm 0.03$ \\
Equiv. $\sigma$ & $\ldots$ & $1.7 \pm 0.2$ \\
$\log S^{\text {mod }}$ & $-0.5 \pm 0.2$ & $-0.6 \pm 0.2$ \\
$p\left(S>S^{\text {mod }}\right)$ & $0.11 \pm 0.07$ & $0.15 \pm 0.09$ \\
Equiv. $\sigma$ & $1.6 \pm 0.4$ & $1.4 \pm 0.4$ \\
\hline \hline
\end{tabular}

Marginalized parameter constraints and tension metrics for both data combination and model comparison are reported in Table III. 

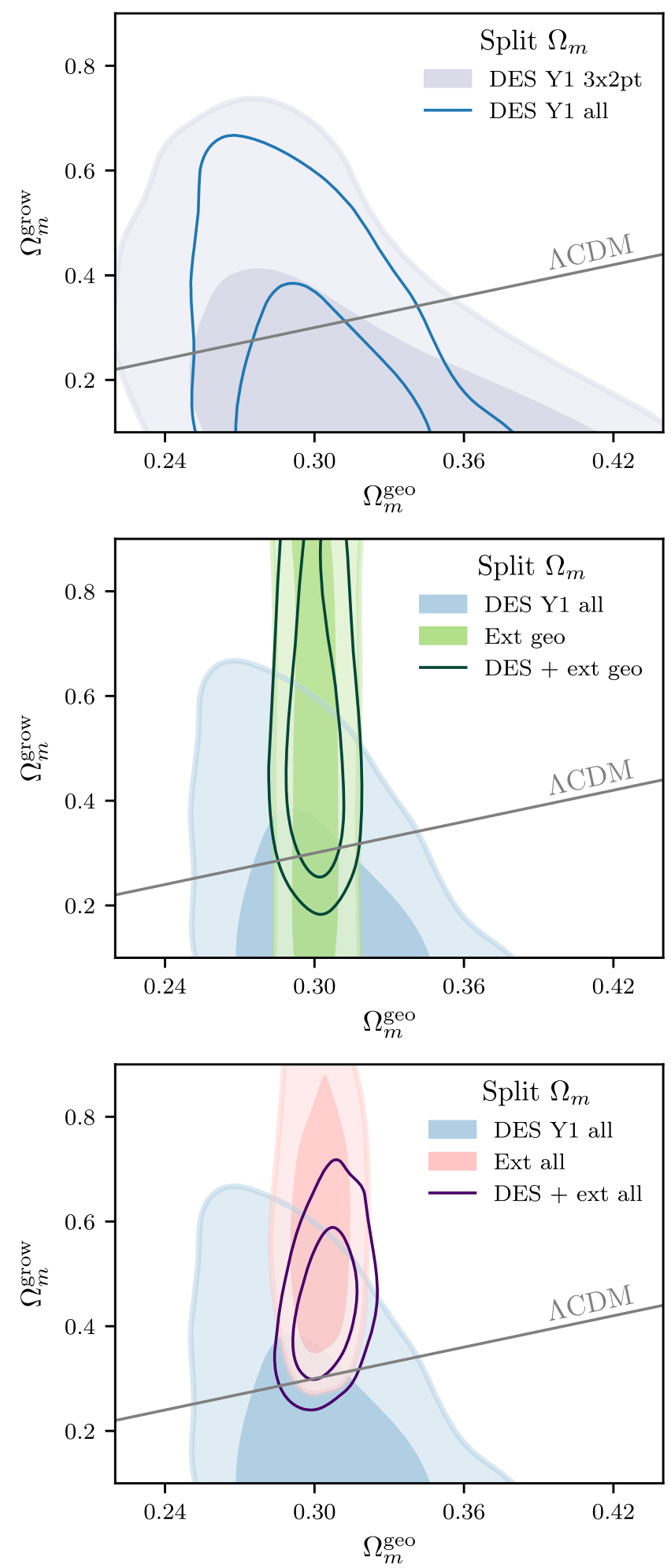

FIG. 3. The $68 \%$ and $95 \%$ confidence regions for $\Omega_{m}^{\text {grow }}$ and $\Omega_{m}^{\mathrm{geo}}$ for our various data combinations. The diagonal gray lines show where $\Omega_{m}^{\text {grow }}=\Omega_{m}^{\text {geo }}$. Note that the three plots have the same axis ranges, and that the vertical axes cover a much larger range of values than the horizontal axes. The blue outline-only contours in the top plot are the same as the shaded blue contours in the other plots.
Looking at DES-only results in the top panel, we find that, as expected, including the (geometric) DES BAO and SNe likelihoods tightens the constraints on $\Omega_{m}^{\text {geo }}$ but only weakly affects $\Omega_{m}^{\text {grow }}$. We find that the constraints on $\Omega_{m}^{\text {geo }}$ are much stronger than those on $\Omega_{m}^{\text {grow }}$ for both the $3 \times 2$ ptonly and the fiducial DES constraints. In fact, the DES constraints on $\Omega_{m}^{\mathrm{geo}}$ are only slightly weaker than $\Lambda \mathrm{CDM}$ constraints on $\Omega_{m}$, implying that most of DES's constraining power is derived from geometric information. This might be surprising, since one might expect a LSS survey to have more growth sensitivity. However, it is consistent with the findings summarized in Ref. [39], which discusses how distance and growth factor measurements can place comparable constraints on the dark energy equation of state when other cosmological parameters are held fixed [37,38], but the growth weakens when one marginalizes over more parameters $[27,35]$. The fact that the confidence regions intersect the $\Omega_{m}^{\text {grow }}=\Omega_{m}^{\text {geo }}$ line but are asymmetrically distributed around it is reflected in the Bayesian suspiciousness measurement of $1.5 \sigma$ tension with $\Lambda$ CDM.

In the middle panel of Fig. 3, we show the combination of the DES data with external geometric measurements from the CMB and BAOs (Ext-geo). As expected, the external geometric data alone put tight constraints on $\Omega_{m}^{\text {geo }}$ but do not constrain $\Omega_{m}^{\text {grow }}$ at all. The combined constraints on $\Omega_{m}^{\text {geo }}$ are straightforwardly dominated by those from the external data, while the DES + Ext-geo constraints on $\Omega_{m}^{\text {grow }}$ are counterintuitively bounded from below but not above. To understand the appearance of the lower bound, note that the DES-only measurement of a given late-time density fluctuation amplitude allows arbitrarily small values of $\Omega_{m}^{\text {grow }}$ because little or no structure growth over time can be compensated by a large primordial amplitude $A_{\mathrm{s}}$. Adding the Planck constraints provides an early-time anchor for $A_{\mathrm{s}}$, and therefore requires $\Omega_{m}^{\text {grow }}$ to be above some minimal value in order to account for the evolution of structure growth between recombination and the redshifts probed by DES. The reason DES's upper bound on $\Omega_{m}^{\text {grow }}$ does not translate to the DES + Ext-geo constraints can also be understood in terms of degeneracies in our model's larger parameter space. We will explore this in more detail in Sec. VI.

Finally, the bottom panel of Fig. 3 shows constraints from DES and Ext-all, which adds BOSS RSD constraints on growth to the previously considered external geometric measurements. We see that, compared to the middle panel's Ext-geo results, adding RSD allows Ext-all to place a lower bound on $\Omega_{m}^{\text {grow }}$, and when combined with DES, $\Omega_{m}^{\text {grow }}$ is bounded on both sides. The fact that there is not very much overlap between the DES and Ext-all contours, with Ext-all preferring somewhat higher $\Omega_{m}^{\text {grow }}$ than DES, reflects their weak $2 \sigma$ tension. The shape of the Ext-all constraints here, 
as well as how DES adds information, is related to a degeneracy between $\Omega_{m}^{\text {grow }}$ and $\sum m_{\nu}$, which we will discuss further in Sec. VI.

\section{B. Splitting $\boldsymbol{\Omega}_{m}$ and $\boldsymbol{w}$}

Figure 4 shows the $68 \%$ and $95 \%$ confidence contours when splitting both $\Omega_{m}$ and $w$ for DES + Ext-all constraints, showing the parameters $\Omega_{m}^{\text {geo }}, \Omega_{m}^{\text {grow }}, w^{\text {geo }}$, and $w^{\text {grow }}$. The most notable feature is the strong degeneracy between the two growth parameters, $\Omega_{m}^{\text {grow }}$ and $w^{\text {grow }}$. We interpret this to mean that while DES + Ext-all can separately constrain growth and geometry, the data cannot distinguish between $\Omega_{m}$-like and $w$-like deviations from the structure growth history expected from $w$ CDM. This behavior is also consistent with the finding of Ref. [26] that, for a given $\Omega_{m}(z), w$ only weakly affects growth rates. This makes it unsurprising that it is difficult to robustly constrain $w^{\text {grow }}$ separately from $\Omega_{m}^{\text {grow }}$.

Because of this degeneracy, even using our most informative "DES + Ext-all" data combination, $w^{\text {grow }}$ is unconstrained, and the upper and lower bounds placed on $\Omega_{m}^{\text {grow }}$ are entirely dependent on the choice of prior for $w^{\text {grow }}$. As discussed in Appendix B, our analyses of simulated data show that projection effects associated with this degeneracy significantly affect the one-dimensional marginalized constraints on both $\Omega_{m}^{\text {grow }}$ and $w^{\text {grow }}$. Because of this, we do not report parameter constraints for this model.

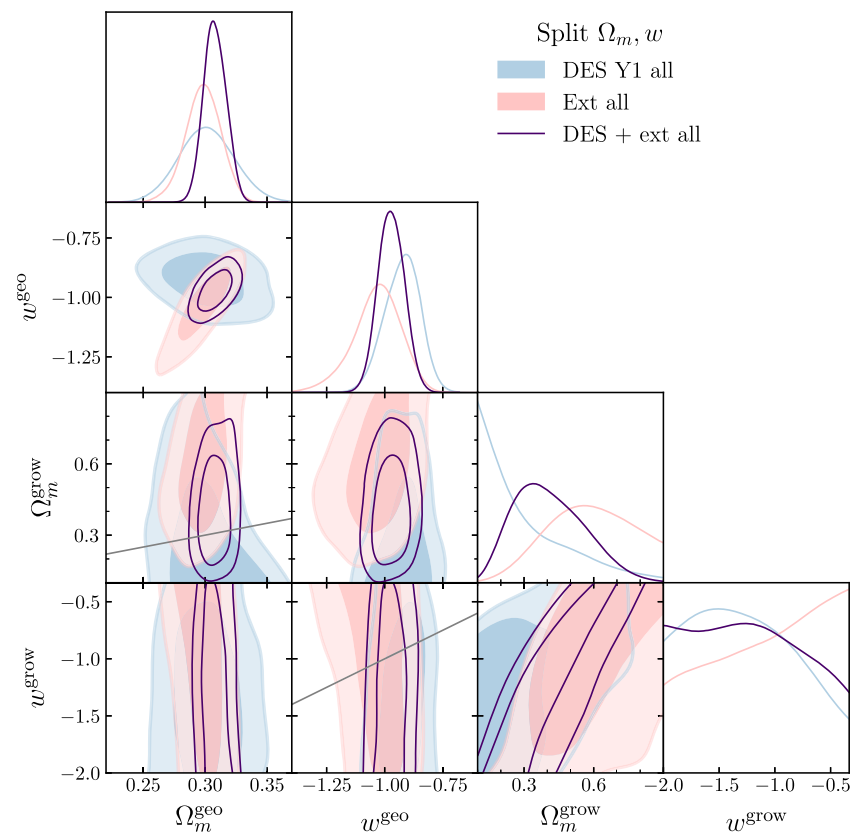

FIG. 4. Marginalized constraints from DES and external data when both $\Omega_{m}$ and $w$ are split. The diagonal panels show normalized one-dimensional marginalized posteriors, while the off-diagonal panels show $68 \%$ and $95 \%$ confidence regions. Solid gray lines show the $w$ CDM parameter subspace where $\Omega_{m}^{\text {grow }}=\Omega_{m}^{\text {geo }}$ and $w^{\text {grow }}=w^{\text {geo }}$.

\section{Consistency with $\Theta^{\text {grow }}=\Theta^{\text {geo }}$}

Ultimately, the question we would like to ask is whether the results above are consistent with $\Lambda \mathrm{CDM}$, or with $w \mathrm{CDM}$, in the case where we split both $\Omega_{m}$ and $w$. There are several ways we can assess this. We begin simply by looking at the two-dimensional confidence regions shown in Figs. 3 and 4, noting whether or not they intersect the lines corresponding to $\Lambda \mathrm{CDM}$ (in Fig. 3 ) and $w C D M$ (in Fig. 4). We see that when we split $\Omega_{m}$, the $68 \%$ confidence intervals for DES and DES + Ext-geo intersect the $\Omega_{m}^{\text {grow }}=\Omega_{m}^{\text {geo }}$ line, while that of DES + Ext-all just touches the $\Lambda \mathrm{CDM}$ line, preferring $\Omega_{m}^{\text {grow }}>\Omega_{m}^{\text {geo }}$. When we split both $\Omega_{m}$ and $w$, both the $\Omega_{m}^{\text {grow }}=\Omega_{m}^{\text {geo }}$ and $w^{\text {grow }}=w^{\text {geo }}$ lines go directly through the DES + Ext-all $68 \%$ confidence intervals.

To assess consistency with $\Theta^{\text {grow }}=\Theta^{\text {geo }}$ in our full parameter space, we use Bayesian suspiciousness $S^{\text {mod }}$, as described in Eq. (30) of Sec. IV B. As we did when we used suspiciousness to evaluate concordance between datasets, we use $p\left(S>S^{\bmod }\right)$ to report the probability to exceed the observed suspiciousness, and we use " 1 -tail equiv. $\sigma$ " as the number of normal-distribution standard deviations with equivalent probability. Here, a larger $S^{\text {mod }}$, smaller $p\left(S>S^{\text {mod }}\right)$, and larger $\sigma$ indicate more tension with $\Theta^{\text {grow }}=\Theta^{\text {geo }}$. Numbers for all of these quantities are shown in Table III. According to this metric, when we split $\Omega_{m}$, we find the DES-only results to have a $1.5 \sigma$ tension with $\Lambda$ CDM. This becomes $1.9 \sigma$ for DES + Ext-geo and $1.0 \sigma$ for DES + Ext-all. When we split both $\Omega_{m}$ and $w$, we find tensions with $w \mathrm{CDM}$ to be $1.6 \sigma$ for DES-only and $1.4 \sigma$ for DES + Ext-all.

As another way of quantifying compatibility of the split $-\Omega_{m}$ constraints with $\Lambda \mathrm{CDM}$, in Fig. 5 we show the marginalized posterior for the difference $\Omega_{m}^{\text {grow }}-\Omega_{m}^{\text {geo }}$. When we assess the fraction of the posterior volume above and below 0 , we find that the fraction of the posterior

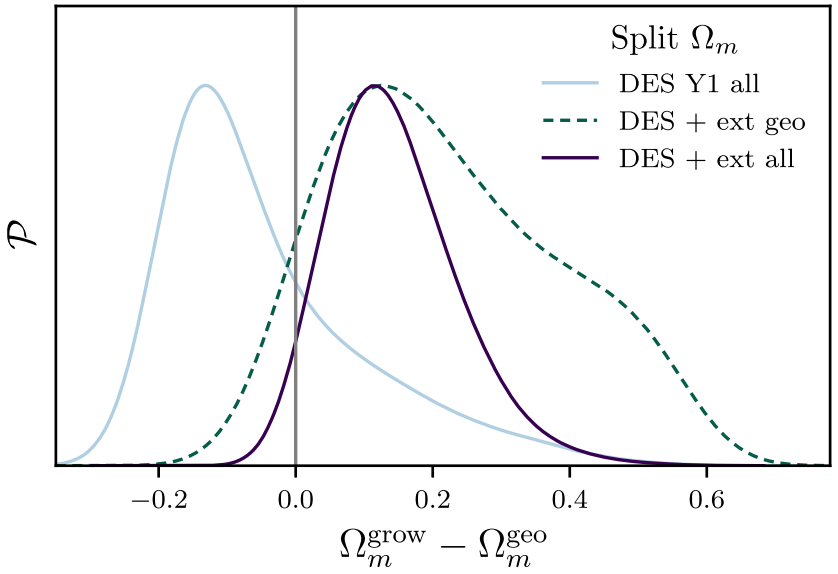

FIG. 5. Marginalized posterior of the difference $\Omega_{m}^{\text {grow }}-\Omega_{m}^{\text {geo }}$, from fitting the split- $\Omega_{m}$ model to the DES, DES + Ext-geo, and DES + Ext-all data combinations. 
volume with $\Omega_{m}^{\text {grow }}>\Omega_{m}^{\text {geo }}$ is $30 \%$ for DES-only, equivalent to a normal-distribution single-tail probability of $0.5 \sigma$. These numbers become $91 \%$ (1.3 $\sigma$ ) for DES + Ext-geo, and $95 \%(1.6 \sigma)$ for DES + Ext-all.

We note two points of caution in interpreting the $\Omega_{m}^{\text {grow }}-$ $\Omega_{m}^{\mathrm{geo}}$ marginalized posterior. First, because of the difference in constraining power on $\Omega_{m}^{\text {grow }}$ and $\Omega_{m}^{\text {geo }}$, there is some asymmetry expected in these marginalized posteriors even if the data are consistent with $\Lambda \mathrm{CDM}$. This can be seen in Fig. 12 of Appendix C, which shows versions of this plot for synthetic data generated with $\Omega_{m}^{\text {grow }}=\Omega_{m}^{\text {geo }}$. Additionally, the posterior distribution is impacted by the priors on $\Omega_{m}^{\text {grow }}$ and $\Omega_{m}^{\text {geo }}$. While the GetDist software allows us to correct for the impact of hard prior boundaries for the parameters we sample over, it is unable to do so for derived parameters. This means that in cases where the shape of the posterior is influenced by the prior boundary of, e.g., $\Omega_{m}^{\text {grow }}$, this will necessarily affect the shape of the marginalized posterior for $\Omega_{m}^{\text {grow }}-\Omega_{m}^{\text {geo }}$. Accounting for these caveats and comparing to the simulated results in Appendix $\mathrm{C}$, we see that the DES + Ext-all probability distribution is shifted to higher $\Omega_{m}^{\text {grow }}-\Omega_{m}^{\text {geo }}$ than was found in simulated analyses. The DES-only and DES + Ext-geo distributions do not appear to be significantly different from what might be expected given parameterspace projection effects in $\Lambda \mathrm{CDM}$.

\section{Summary of main results}

The results discussed in this section are summarized in Table III. In the table, for the split $\Omega_{m}$ model we show onedimensional marginalized constraints on $\Omega_{m}^{\text {grow }}$ and $\Omega_{m}^{\text {geo }}$ from DES + Ext-geo and DES + Ext-all, along with $\Lambda \mathrm{CDM}$ and $w \mathrm{CDM}$ constraints for comparison. For each parameter, we show two-sided errors corresponding to the $68 \%$ confidence interval for the one-dimensional marginalized posterior. Because we expect the one-dimensional marginalized posteriors to be subject to significant projection effects for DES-only constraints on the split $\Omega_{m}$ model and for the DES + Ext-all constraints when splitting both $\Omega_{m}$ and $w$, as discussed in Sec. IV and Appendix B, we do not report parameter bounds for those cases.

For all model-data combinations considered, we use Bayesian suspiciousness as defined in Sec. IV B to report data tension and model comparison statistics. In Table III, $\tilde{d}$ is the Bayesian model dimensionality [Eq. (25)], quantifying the effective number of parameters constrained; $S^{\text {dat }}$ is the Bayesian suspiciousness, assessing agreement between pairs of datasets [Eq. (24)]; and $S^{\text {mod }}$ is the modelcomparison Bayesian suspiciousness [Eq. (30)], quantifying tension or agreement with $\Theta^{\text {grow }}=\Theta^{\text {geo }}$. The quantities $p\left(S>S^{X}\right)$, for $X \in[$ dat, mod ], give the probability that a random realization exceeds the observed suspiciousness $S^{X}$, and "equiv. $\sigma$ " translates that probability into the number of standard deviations with an equivalent double-tail probability for a normal distribution [Eq. (33)]. Large $S$, small $p$, and large equivalent $\sigma$ indicate tension, while small $S$, large $p$, and small equivalent $\sigma$ indicate concordance. For all quantities, the numbers quoted in Table III are the mean and standard deviation from sampling error reported by ANESTHETIC.

As an alternative model-comparison statistic for the split$\Omega_{m}$ model, we additionally report $p\left(\Omega_{m}^{\text {grow }}>\Omega_{m}^{\text {geo }}\right)$, the fraction of the posterior volume with $\Omega_{m}^{\text {grow }}>\Omega_{m}^{\text {geo }}$. For this part of the table, the "equiv. $\sigma$ " is the number of normaldistribution standard deviations with equivalent single-tail probability.

\section{RESULTS: IMPACT OF GROWTH-GEOMETRY SPLIT ON OTHER PARAMETERS}

Here we explore how our split parametrization, focusing on splitting only $\Omega_{m}$, affects the inference of other cosmological parameters. In this discussion, we will primarily reference Fig. 6, which shows two-dimensional marginalized posteriors of DES + Ext-all constraints on $\Omega_{m}^{\text {grow }}, \Omega_{m}^{\text {geo }}$, the difference $\Omega_{m}^{\text {grow }}-\Omega_{m}^{\text {geo }}, \sum m_{\nu}$, $S_{8} \equiv \sigma_{8} \sqrt{\Omega_{m}^{\text {geo }} / 0.3}, h$, and $A_{\text {IA }}$. For comparison, we also show a DES + Ext-geo version of this plot in Fig. 17 in Appendix E. We use this higher-dimensional visualization of the posterior to characterize how additional degrees of freedom in the relationship between expansion history and structure growth change considerations in cosmological analyses, both in terms of how we model astrophysical effects $\left(\sum m_{\nu}, A_{\mathrm{IA}}\right)$ and in terms of commonly studied tensions $\left(S_{8}, h\right)$.

In the off-diagonal panels of Fig. 6, 68\% and 95\% confidence regions are shown for DES-only as blue shaded contours, for Ext-all as pink shaded contours, and for the combination DES + Ext-all as dark purple outlines. The diagonal panels show normalized one-dimensional marginalized posteriors for each parameter. Solid gray lines show the $\Lambda \mathrm{CDM}$ subspace where $\Omega_{m}^{\text {grow }}=\Omega_{m}^{\text {geo }}$, and gray dashed lines show the DES + Ext-all posterior for $\Lambda$ CDM.

\section{A. Effect of split on neutrino mass}

Because the combination of Planck, BOSS BAO, and BOSS RSD data is able to tightly constrain cosmological parameters in $\Lambda \mathrm{CDM}$, it may be surprising that DES adds information at all when combined with the Ext-all data. Looking at Fig. 6, we see that it does so because the external data exhibits a significant degeneracy between $\Omega_{m}^{\text {grow }}$ and the sum of neutrino masses $\sum m_{\nu}$. The Ext-all degeneracy occurs because changes in $\sum m_{\nu}$ and $\Omega_{m}^{\text {grow }}$ have competing effects on the matter power spectrum: higher neutrino mass suppresses structure formation at small scales $\left(k \gtrsim 10^{-2} h \mathrm{Mpc}^{-1}\right)$, while raising $\Omega_{m}^{\text {grow }}$ results produces more late-time structure. DES data adds constraining power because it provides an upper bound on $\Omega_{m}^{\text {grow }}$, which breaks that degeneracy. 


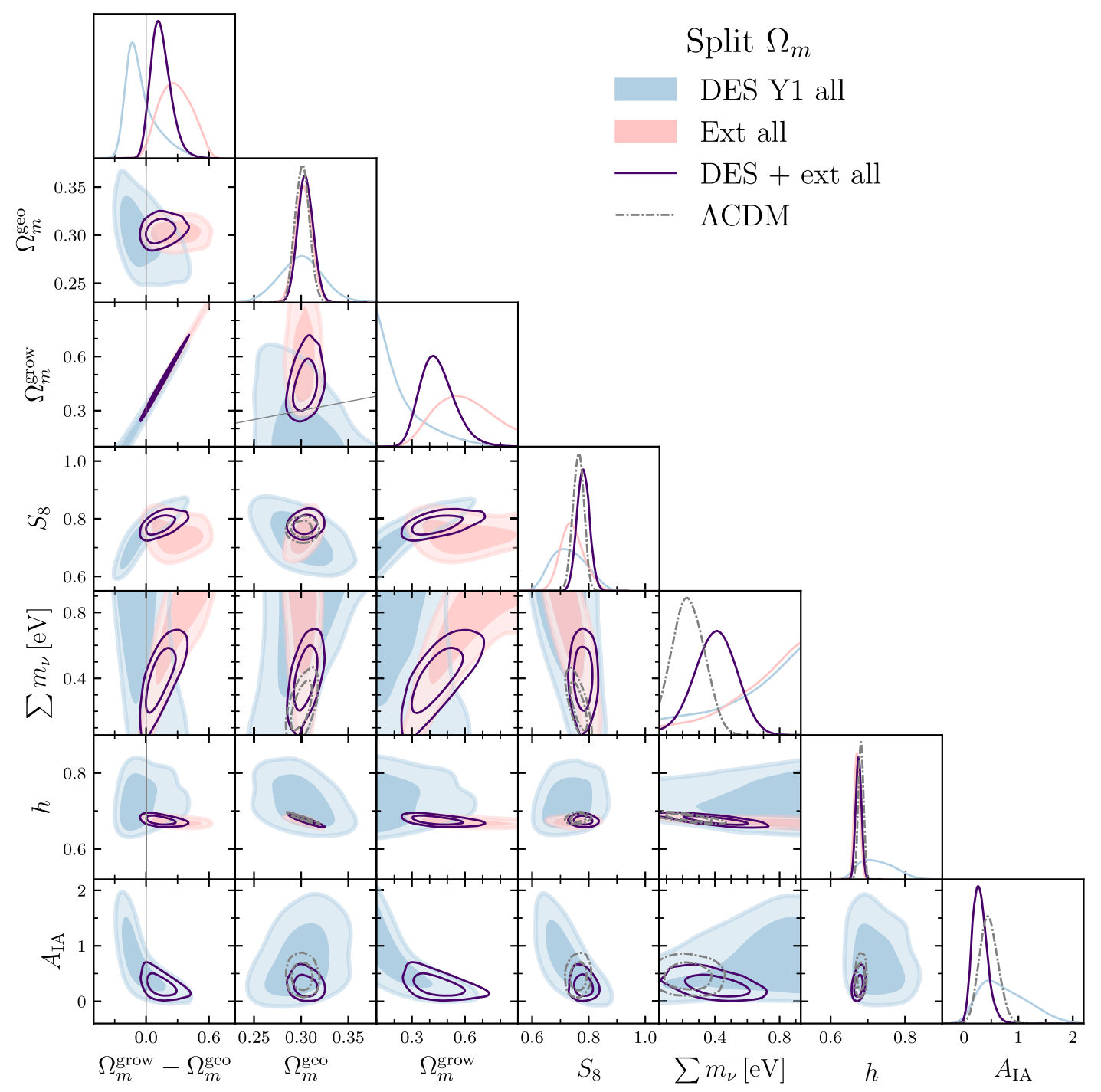

FIG. 6. Constraints from the DES and the Ext-all external dataset, which includes the compressed Planck likelihood, BOSS DR12 BAO, and BOSS DR12 RSD. The off-diagonal panels show the $68 \%$ and $95 \%$ confidence intervals for each data combination, while the diagonal panels show normalized one-dimensional marginalized posteriors on parameters. DES-only results are shown in blue, Ext-all results are pink, and their combination is shown using unshaded purple contours. The gray dashed curves show DES + Ext-all constraints in $\Lambda \mathrm{CDM}$, and the gray solid lines show where $\Omega_{m}^{\text {grow }}=\Omega_{m}^{\text {geo }}$.

Looking at the marginalized constraints on $\sum m_{\nu}$, we see that both DES + Ext-all (Fig. 6) and DES + Ext-geo (Fig. 17) constraints produce a detection of neutrino mass at $\sum m_{\nu}=0.4 \pm 0.1 \mathrm{eV}$, which is significantly higher than the upper bounds obtained from the combined analysis of BOSS DR12 and the full Planck temperature and polarization power spectra [49,57]. The DES-only posterior gives a weak lower bound on neutrino mass, though we suspect that this may be at least in part caused by parameter-space projection effects. In $\Lambda \mathrm{CDM}$, the Ext-all constraints on $\sum m_{\nu}$ become an upper bound of $\sum m_{\nu}<0.45 \mathrm{eV}$ at 95\% confidence, which is consistent with the BOSS results (though weaker because we do not use the full Planck likelihood), while the DES preference for high $\sum m_{\nu}$ remains. This causes the DES + Ext-all $\Lambda$ CDM posterior, shown as a gray dashed line in Fig. 6, to peak at $\sum m_{\nu}=0.2 \pm 0.1 \mathrm{eV}$.

To begin interpreting the preference for high $\sum m_{\nu}$, we can look at the $\sum m_{\nu}-\Omega_{m}^{\text {grow }}$ panel of Fig. 6 and note that the Ext-all constraints exhibit a preference for the high$\sum m_{\nu}$, high- $\Omega_{m}^{\text {grow }}$ part of parameter space. That preference combined with the DES upper bound on $\Omega_{m}^{\text {grow }}$ likely drives the $2 \sigma$ tension between Ext-all and DES, and it appears to be responsible for pulling the combined DES + Ext-all constraints away from the $\Lambda \mathrm{CDM} \Omega_{m}^{\text {grow }}=\Omega_{m}^{\text {geo }}$ line.

It is instructive to examine how the constituent Planck and BOSS likelihoods combine to produce the Ext-all contours. We show this in Fig. 7, with the compressed Planck posterior in yellow, BOSS $\mathrm{BAO}+\mathrm{RSD}$ in orange, and their combination, Ext-all, as black outlines. 


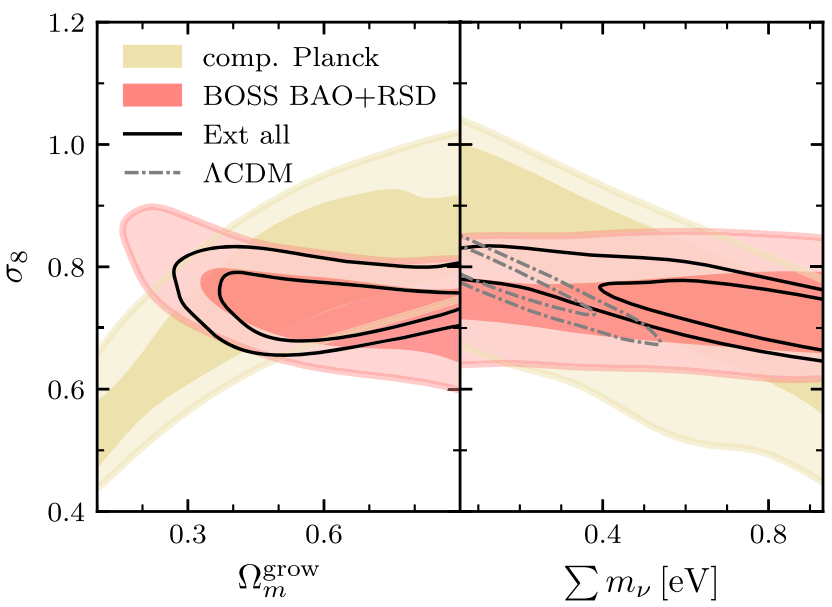

FIG. 7. Constraints on parameters most relevant for describing late-time growth, shown for the datasets that make up Ext-all. Contours show the $68 \%$ and $95 \%$ confidence regions for the compressed Planck likelihood in yellow, and those for BOSS DR12 BAO and RSD in orange. The unshaded black contours correspond to Ext-all, and are the same as the pink contours in other figures. Gray dashed contours show $\Lambda \mathrm{CDM}$ results for Ext-all.

The compressed Planck likelihood approximately defines a plane in the $\sigma_{8}-\sum m_{\nu}-\Omega_{m}^{\text {grow }}$ parameter space, because Planck's measurement of $A_{\mathrm{s}}$ can be extrapolated forward to predict $\sigma_{8}$, but the effects of $\Omega_{m}^{\text {grow }}$ and $\sum m_{\nu}$ on late-time structure growth loosen that predictive relationship. The BOSS data probe late-time structure more directly, so the combined $\mathrm{BAO}$ and RSD results can be thought of as roughly providing a measurement of $\sigma_{8}$ that is insensitive to $\sum m_{\nu}$ and only weakly dependent on $\Omega_{m}^{\text {grow }}$.

Putting all of this together, we see that the shape of the Ext-all posterior strongly depends on the relationship between Planck's measurement of $A_{\mathrm{s}}$ and BOSS's measurement of $\sigma_{8}$, as well as the extent to which late-time degrees of freedom impact how deterministically Planck's $A_{\mathrm{s}}$ constraint maps to $\sigma_{8}$. For example, if the Planck $A_{\mathrm{s}}$ constraints were lowered slightly, or the BOSS $\sigma_{8}$ constraints were raised, this would move the Ext-all constraints towards lower $\sum m_{\nu}$ and consequently, lower $\Omega_{m}^{\text {grow }}$. The DES + Ext-geo constraints have a similar property: we can see in the $S_{8}-\sum m_{\nu}$ panel of Fig. 17 that slight relative changes to the Planck $A_{\mathrm{s}}$ or DES $S_{8}$ constraints can have a significant impact on the $\sum m_{\nu}$ posterior. In other words, our results' preference for high $\sum m_{\nu}$ (and consequently, high $\Omega_{m}^{\text {grow }}$ ) can be interpreted as a manifestation of the early- versus late-Universe $\sigma_{8}$ tension discussed in the Introduction.

Our findings here are in line with several previous studies which report a preference for $\sum m_{\nu} \sim 0.3 \mathrm{eV}$ when modeling degrees of freedom affecting structure growth are introduced to combined CMB and LSS analyses. These include the growth-geometry split analysis of Refs. [41,42], as well as examinations of neutrino mass in conjunction with $A_{\text {Lens }}[132,133]$ (which describes the amount of lensing-induced smoothing of the CMB power spectrum), time-dependent dark energy [134], and modified gravity [135]. Notably, however, these results are in contrast with those documented in Fig. 19 of the official BOSS DR12 analysis paper [49], which show that BOSS DR12 BAO and RSD data combined with Planck temperature and polarization are able to constrain $\sum m_{\nu}<0.25 \mathrm{eV}$ at 95\% confidence, even when marginalizing over $A_{\text {Lens }}$ and a free amplitude multiplying $f \sigma_{8}$. Our Ext-all constraints are weaker than this, because using a compressed Planck likelihood causes us to lose information about a degeneracy between $\sum m_{\nu}$ and the shift parameter $R$ that is present in the full likelihood (which in the BOSS analysis is broken by $\mathrm{BAO}$ angular diameter distance measurements), and potentially also because our choice of priors requires $\sum m_{\nu}>0.06 \mathrm{eV}$, while BOSS uses $\sum m_{\nu}>0 \mathrm{eV}$.

To explore how our results would be affected by tighter $\sum m_{\nu}$ constraints, in Fig. 8 we show DES + Ext-geo and DES + Ext-all constraints on $\Omega_{m}^{\text {grow }}$ and $\Omega_{m}^{\text {geo }}$ when the sum of neutrino masses is fixed to its minimal value, $0.06 \mathrm{eV}$. Additionally, in Sec. 2 of Appendix E, Fig. 18 shows how either fixing $\sum m_{\nu}$ or requiring $\Omega_{m}^{\text {grow }}=\Omega_{m}^{\text {geo }}$ alters the Extall constraints (without DES data), and Table IV reports fixed- $\sum m_{\nu}$ versions of our data and model tension metrics. We find that assuming minimal neutrino mass allows us to constrain $\Omega_{m}^{\text {grow }}$ with either DES + Ext-geo alone or just the Ext-all data, and that the fixed-neutrino-mass DES + Ext-all constraints are dominated by information from the external data. For all data combinations, fixing neutrino mass improves the agreement between datasets, and the split $-\Omega_{m}$ constraints become consistent with $\Lambda \mathrm{CDM}$ at the $<1 \sigma$ level.

\section{B. Effect of split on $S_{8}$}

In examining the effect of the growth-geometry split parametrization on $\sigma_{8}$, we can orient ourselves by making a few observations. First, as noted in Sec. II B, the usual negative degeneracy between $\Omega_{m}$ and $\sigma_{8}$ seen in weaklensing analyses appears in the DES-only constraints here as a degeneracy between $\Omega_{m}^{\text {geo }}$ (which appears in the lensing prefactor of the lensing kernel) and $\sigma_{8}$. Thus, to more easily compare to results in other papers, in Fig. 6 we show constraints on $S_{8}=\sigma_{8} \sqrt{\Omega_{m}^{\text {geo }} / 0.3}$.

In contrast, the DES-only constraints on $\Omega_{m}^{\text {grow }}$ and $\sigma_{8}$ are positively correlated. This might seem counterintuitive, because changing $\Omega_{m}^{\text {grow }}$ and changing $A_{\mathrm{s}}$ have similar effects on the matter power spectrum, and we are used to thinking of $\sigma_{8}$ as equivalent to $A_{\mathrm{s}}$. However, it is important to remember that splitting growth and geometry breaks our usual intuition about the one-to-one relationship between $A_{\mathrm{s}}$ and $\sigma_{8}$. While $\Omega_{m}^{\text {grow }}$ and $A_{\mathrm{s}}$ do indeed have a negative degeneracy (see Fig. 10), $\Omega_{m}^{\text {grow }}$ and $\sigma_{8}$ do not. Because $\sigma_{8}$ 

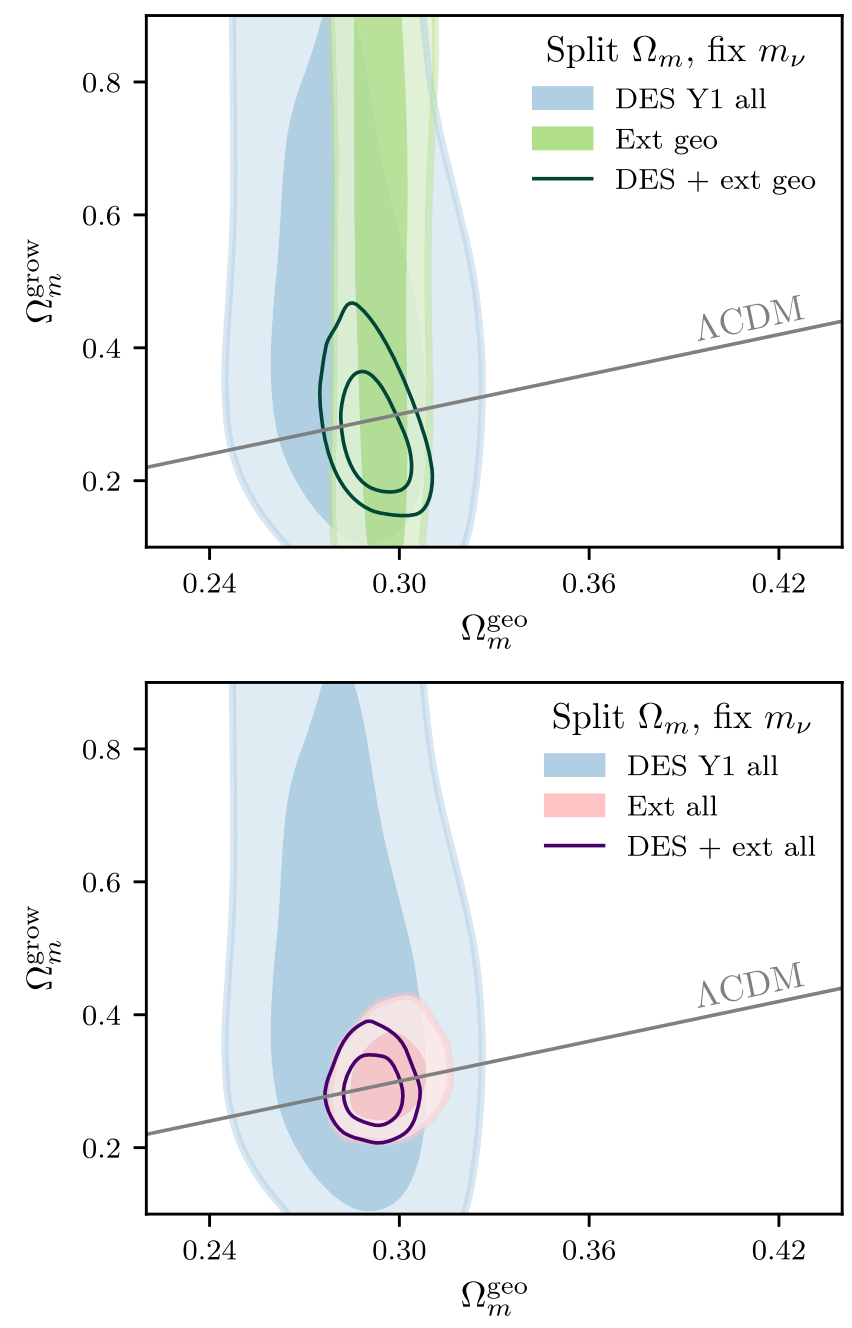

FIG. 8. The same combined DES + Ext-geo (top) and DES + Ext-all (bottom) constraints as the second and third panels of Fig. 3, but with the sum of neutrino masses fixed to $0.06 \mathrm{eV}$.

is a derived parameter obtained by integrating the power spectrum, and increasing $\Omega_{m}^{\text {grow }}$ raises the amplitude of the power spectrum, if all other parameters are fixed, raising $\Omega_{m}^{\text {grow }}$ will produce an increase in $\sigma_{8}$. Thus, the degeneracy we find between $\Omega_{m}^{\text {grow }}$ and $\sigma_{8}$ is expected for the same reason that we generally expect a positive correlation between $A_{\mathrm{s}}$ and $\sigma_{8}$.

Compared to $\Lambda \mathrm{CDM}$, splitting $\Omega_{m}$ has relatively little effect on the DES + Ext-all $S_{8}$ constraints, while it weakens and shifts those from DES and DES + Ext-geo. These $S_{8}$ values remain consistent with the DES Y1-3 $\times 2 \mathrm{pt}$ $\Lambda \mathrm{CDM}$ result of $0.773_{-0.020}^{+0.026}$ and below the full Planck $\Lambda \mathrm{CDM}$ constraint of $0.842_{-0.025}^{+0.027}$ [3]. We note that the DES + Ext-geo constraint on $S_{8}$ moves upwards enough to be consistent with the $\Lambda$ CDM Planck result, while the DES estimate moves further away_albeit, more by reducing the lower bound than by ruling out values preferred by DES $\Lambda \mathrm{CDM}$. This behavior is qualitatively similar to the $S_{8}$ constraint in the DES Y1-3 $\times 2$ pt for the $\Sigma-\mu$ modified gravity model analysis of Ref. [30].

We find no disagreement between DES and the external data's marginalized $S_{8}$ constraints, which might suggest that our growth-geometry split is able to resolve the $\sigma_{8}$ tension. However, this interpretation is confounded by the fact that we use more limited information from Planck than standard analyses. The compressed Planck likelihood we use does not contain any information from the lensing smoothing of CMB power spectrum peaks, and it is unable to constrain neutrino mass on its own, so it exhibits a negative $S_{8^{-}} \sum m_{\nu}$ degeneracy. This causes the DES and Ext-geo marginalized posteriors on $S_{8}$ to be compatible even in $\Lambda C D M$. In other words, we can concretely say that the Planck preference for high $S_{8}$ compared to DES and other probes of LSS relies on information from the CMB that is not included in the geometry-only compressed likelihood.

\section{Effect of split on $\boldsymbol{H}_{\mathbf{0}}$}

We find that in our split parametrization, the constraints on $h \equiv H_{0} /\left(100 \mathrm{~km} \mathrm{~s}^{-1} \mathrm{Mpc}^{-1}\right)$ do not significantly change relative to what they are in $\Lambda$ CDM. The Planck likelihood provides nearly all the information on $h$, with its $\Omega_{m} h^{2}$ constraint manifesting as a tight ellipse in the $\Omega_{m}^{\text {geo }}-h$ planes of both Figs. 6 and 17. This suggests that nonstandard structure growth will have little impact on the value of the Hubble constant inferred from the data we consider, and therefore that it is a poor candidate for resolving the $H_{0}$ tension.

\section{Effect of split on $A_{\mathrm{IA}}$}

Finally, we examine how opening up our split parameter space impacts constraints on the amplitude of intrinsic alignments. We can see in Fig. 6 that there is a significant negative degeneracy between $\Omega_{m}^{\text {grow }}$ and $A_{\text {IA }}$ present in the DES posterior. This occurs because the factor of $\rho_{m 0}$ in Eq. (6) makes the contribution of intrinsic alignments signal proportional to the product $\Omega_{m}^{\text {grow }} A_{\mathrm{IA}}$. As we discuss in Sec. IV A and Appendix B, we believe this degeneracy is why our growth-focused beyond- $\Lambda \mathrm{CDM}$ parametrization is more sensitive to assumptions about the redshift dependence of intrinsic alignments than the other extensions to $\Lambda \mathrm{CDM}$ considered in Ref. [30].

Like the previous DES Y1 papers $[3,136,137]$, we are not able to constrain the redshift power-law slope $\alpha_{I A}$, but we are able to constrain the amplitude $A_{\mathrm{IA}}$. For comparison, in $\Lambda \mathrm{CDM}$, our DES, DES + Ext-geo, and DES + Ext-all posteriors all give $A_{\mathrm{IA}}=0.4 \pm 0.2$, which is compatible with (and about twice as constraining as) what is reported in Ref. [3] for DES Y1-3 $\times 2$ pt. When we split $\Omega_{m}$, the $\Omega_{m}^{\text {grow }}-A_{\text {IA }}$ degeneracy causes the DES-only constraints to widen considerably, with the bulk of the posterior volume residing in the region with small $\Omega_{m}^{\text {grow }}$ and high $A_{\mathrm{IA}}$. 
Combining external data places a lower bound on $\Omega_{m}^{\text {grow }}$, which breaks the degeneracy and restricts $A_{\mathrm{IA}}$ to small values. In fact, DES + Ext-geo and DES + Ext-all constraints on $A_{\mathrm{IA}}$ are slightly tighter and peak at slightly lower values in our split parametrization compared to $\Lambda \mathrm{CDM}$. We observe a slight negative degeneracy between $A_{\mathrm{IA}}$ and $\sum m_{\nu}$ in these combined posteriors, so it is possible that this is caused by the same properties of the data which drive the high- $\sum m_{\nu}$ results.

\section{DISCUSSION}

We perform a combined analysis of DES Y1 galaxy clustering and weak lensing, DES Y1 BAO, and DES Y3 supernova measurements in which we split cosmological parameters related to the physics of dark energy into separate "growth" and "geometry" versions. In this growthgeometry split analysis, the geometry parameters $\Theta^{\text {geo }}$ enter model predictions for observables related to expansion history, including all distances, the shape of the high- $z$ matter power spectrum, and projection operations used to convert the three-dimensional power spectrum to observed 2PCF. The growth parameters, in turn, enter calculations of late-time structure growth: $\Theta^{\text {grow }}$ are used to compute the linear and nonlinear evolution of the matter power spectrum at late times as well as intrinsic alignment contributions to shear correlations.

We primarily focus on splitting $\Omega_{m}$, and our main results are reported based on two data combinations: DES + Ext-geo, which combines the DES measurements with external geometric information from BOSS DR12 BAO and a compressed Planck 2015 likelihood, and DES + Ext-all, which additionally includes BOSS DR12 RSD measurements as an external probe of structure growth. To supplement these main results, we also consider secondary data-model combinations which are less robust to changes in our modeling assumptions but can still aid in the interpretation of the main results: DES-only constraints on split $\Omega_{m}$, and DES + Ext-geo constraints when splitting both $\Omega_{m}$ and $w$. We stress-test our analysis procedure by ensuring that the results are not biased in the presence of a sample of injected systematic errors, and perform a blinded analysis; see Sec. IV A for details.

We use these analyses to address the questions raised in the Introduction, which we now answer in order.

\section{A. Are DES constraints informed more by growth or geometric information?}

For all data combinations considered, we find constraints on geometric parameters to be much tighter than those on growth parameters (Fig. 3). Thus, at least in the context of how we have defined growth and geometric observables, DES constraints are more informed by geometry than by a direct measurement of the evolution of structure growth. This is both because changing $\Omega_{m}^{\text {grow }}$ has a smaller effect on the matter power spectrum than changing $\Omega_{m}^{\text {geo }}$ (see Fig. 1), and because of parameter degeneracies impacting the $\Omega_{m}^{\text {grow }}$ constraints. As seen in Fig. 1, changing $\Omega_{m}^{\text {grow }}$ results in a nearly scale-independent amplitude change to $P(k)$. Consequently, $\Omega_{m}^{\text {grow }}$ will be largely degenerate with any parameters that change the amplitude of the DES signal. In the case of the lensing information, which contributes significantly to the overall constraints, the amplitude is controlled by $S_{8}$, and is largely degenerate with the neutrino mass and intrinsic alignment parameters. Consequently, degeneracies between these parameters and $\Omega_{m}^{\text {grow }}$ degrade the $\Omega_{m}^{\text {grow }}$ constraints.

In fact, it is only when we combine two independent measures of structure growth, in the DES + Ext-all data combination, that we are able to fully constrain $\Omega_{m}^{\text {grow }}$. For both DES + Ext-geo (where growth information comes only from DES) and Ext-all (where growth information comes only from BOSS RSD), we see that constraints on late-time structure growth are limited by our inability to break parameter degeneracies. The Ext-all measurements do not constrain $\Omega_{m}^{\text {grow }}$ on their own because of a degeneracy between $\Omega_{m}^{\text {grow }}$ and $\sum m_{\nu}$ (Fig. 6), while DES + Ext-geo is mainly limited by its inability to distinguish between $\Omega_{m}^{\text {grow }}$ and $A_{\text {IA }}$ (Fig. 17). When we combine all of these data together as DES + Ext-all, these degeneracies are broken, and we are able to constrain $\Omega_{m}^{\text {grow }}$. When we fix the sum of neutrino masses to $0.06 \mathrm{eV}$, we find that either Ext-all (with no DES data) or DES + Ext-geo are able to constrain both $\Omega_{m}^{\text {geo }}$ and $\Omega_{m}^{\text {grow }}$ on their own (Fig. 8).

When we split both $\Omega_{m}$ and $w$, a significant degeneracy between $\Omega_{m}^{\text {grow }}$ and $w^{\text {grow }}$ prevents us from being able to constrain the growth parameters even with DES + Ext-all data (Fig. 4). This suggests that additional growth probes would need to be included in order to provide enough redundancy to distinguish between $\Omega_{m}$-like and $w$-like deviations from standard structure growth.

\section{B. Are the data consistent with $\Theta^{\text {grow }}=\Theta^{\text {geo }}$ ?}

Our constraints on $\Theta^{\text {grow }}$ and $\Theta^{\text {geo }}$ are statistically consistent, in the sense that we find tensions with $\Lambda \mathrm{CDM}$ to be less than $2 \sigma$ when assessed using either the marginalized posterior for the difference $\Omega_{m}^{\text {grow }}-\Omega_{m}^{\text {geo }}$, or Bayesian suspiciousness. For both the DES + Ext-geo and DES + Ext-all data combinations, the bulk of our posterior resides in the part of parameter space where $\Omega_{m}^{\text {grow }}>\Omega_{m}^{\text {geo }}$ (Fig. 5). This preference is not seen for DES data alone, where degeneracies with $A_{\mathrm{s}}$ and $A_{\mathrm{IA}}$ prevent one from placing a lower bound on the growth parameter (Fig. 10), shifting the posterior towards low $\Omega_{m}^{\text {grow }}$. Equating the fraction of the posterior volume above the $\Lambda \mathrm{CDM}$ line of $\Omega_{m}^{\text {grow }}=\Omega_{m}^{\text {geo }}$ with equivalent one-sided $p$-values for a Gaussian distribution, we find that the DES-only posterior is in agreement with $\Lambda \mathrm{CDM}$ at the $0.5 \sigma$ level, while DES + Ext-geo and DES + Ext-all are 
consistent with $\Lambda \mathrm{CDM}$ at the $1.3 \sigma$ and $1.6 \sigma$ levels, respectively. Bayesian suspiciousness quantifies the agreement of our posterior with $\Theta^{\text {grow }}=\Theta^{\text {geo }}$ in the model's full parameter space. According to this metric, when we split $\Omega_{m}$, the DES-only posterior has a $1.5 \sigma$ tension with $\Lambda \mathrm{CDM}, \mathrm{DES}+$ Ext-geo's tension is $1.9 \sigma$, and $\mathrm{DES}+$ Ext-all's is $1.0 \sigma$. Fixing $\sum m_{\nu}=0.06 \mathrm{eV}$ brings all three data combinations into $<1 \sigma$ agreement with $\Lambda$ CDM. When we split both $\Omega_{m}$ and $w$, we find a DESonly tension with $w \mathrm{CDM}$ of $1.6 \sigma$ and a DES + Ext-all tension of $1.4 \sigma$. We caution that given the strong degeneracy between $\Omega_{m}^{\text {grow }}$ and $A_{\mathrm{IA}}$, these results will be sensitive to changes in our model of the redshift dependence of intrinsic alignments (Fig. 13).

\section{Is the DES preference for low $\sigma_{8}$ compared to Planck driven more by geometry or growth?}

This question is not straightforward to answer, but our results support the idea that the $S_{8}$ tension is driven by constraints on the evolution of structure, as opposed to a mismatch between DES and Planck geometric constraints which somehow propagates into the $S_{8}$ parameter direction. To explain this conjecture, we note that for all data combinations we consider, constraints on geometry parameters are very similar to their unsplit $\Lambda \mathrm{CDM}$ or $w \mathrm{CDM}$ constraints. This means that our split parametrization can be viewed as a generic way of allowing the properties of structure growth to vary around a fixed $\Lambda$ CDM background. Thus, if splitting growth and geometry absorbs the offset between DES and Planck $\sigma_{8}$ measurements into a deviation from $\Omega_{m}^{\text {grow }}=\Omega_{m}^{\text {geo }}$, this would suggest that modifications to structure growth explain the $\sigma_{8}$ tension. The question therefore becomes: does our split parametrization relieve tension between DES and Planck?

The datasets we consider somewhat complicate this assessment, because we do not analyze the full Planck likelihood that is typically used to quantify the $S_{8}$ tension. The closest comparison we can make is between DES and Ext-geo constraints. The Ext-geo data are less constraining than typical analyses of the full Planck likelihood with $A_{\text {Lens }}=1$, enough so that even in $\Lambda \mathrm{CDM}$ their $1 \sigma$ confidence regions for $\sigma_{8}$ overlap substantially with those from DES. Since we construct the compressed Planck likelihood specifically to be insensitive to late-time structure growth (including by marginalizing over $A_{\text {Lens }}$ ), this could be a clue that it is in fact growth-related CMB observables that drive the Planck preference for high $\sigma_{8}$ relative to DES.

That being said, our results may still contain some indication of the data properties which drive the $\sigma_{8}$ tension in $\Lambda \mathrm{CDM}$. As we discuss in Sec. VI A, our DES + Ext-geo, Ext-all, and DES + Ext-all constraints on $\sum m_{\nu}$ depend sensitively on the relative value of $A_{\mathrm{s}}$ measured by Planck compared to $S_{8}$ measured by either DES or BOSS RSD (Fig. 7). Thus, the fact that those constraints favor high neutrino mass - and consequently, high $\Omega_{m}^{\text {grow }}$-is possibly an indication that the $\mathrm{CMB}$ data prefer a higher density fluctuation amplitude than the LSS observables.

On the topic of tensions, we additionally note that splitting growth and geometry has almost no impact on $H_{0}$ constraints (Figs. 6 and 17). This supports the idea that it is difficult to resolve the Hubble tension with simple model extensions to $\Lambda \mathrm{CDM}$ which alter late-time structure growth, echoing arguments made in Refs. [17,138,139], as well as findings from studies of decaying dark matter $[20,21,140]$, modified gravity, and coupled dark energy models [53,57].

\section{Comparison to previous results}

We now compare our results to those from other geometry-growth analyses in the literature. Direct comparisons are made challenging by the fact that each work made different choices in how to define the geometry-growth split, in addition to using different datasets and applying different modeling of the systematics. Nevertheless, some general conclusions can be drawn from these comparisons.

Our modeling choices are closest to those in Ref. [41]. They combine the CFHTLens weak lensing with an earlyUniverse prior based on Planck 2013 data (which is somewhat comparable to our DES + Ext-geo data combination, which also has additional geometric constraints from supernovae and BAOs), and also include galaxy cluster abundances which are sensitive to both geometry and growth. The fiducial analysis in Ref. [41] is, however, less conservative than ours, as it fixes neutrino mass and does not include any intrinsic alignments in the weak lensing modeling. As a result, even though Ref. [41] uses less constraining data, they constrain $\Omega_{m}^{\text {grow }}$ more tightly than we do; the strength of our $\Omega_{m}^{\text {grow }}$ constraints becomes comparable to theirs when we fix neutrino mass (see Sec. 2 of Appendix E). These differences aside, they agree with us in finding that $\Omega_{m}^{\text {geo }}$ is better constrained than $\Omega_{m}^{\text {grow }}$, that the constraints are compatible with $\Omega_{m}^{\text {grow }}=\Omega_{m}^{\text {geo }}$, and that the majority of the posterior resides in the $\Omega_{m}^{\text {grow }}>\Omega_{m}^{\text {geo }}$ part of parameter space. When splitting both $w$ and $\Omega_{m}$, Ref. [41] finds a $3 \sigma$ preference for $w^{\text {grow }}>w^{\text {geo }}$, strongly indicating less structure than would be expected given constraints from expansion history, though letting $\sum m_{\nu}$ vary entirely removes that tension in favor of high neutrino masses.

References [40,42] perform a "perturbations vs background" split and use growth parameters to compute CMB anisotropy properties, rather than classifying growth parameters as specific to late-time structure evolution as is done in this paper. Therefore, the split- $\Omega_{m}$ model in these references probes different physics and is not directly comparable to our results. However, their results from splitting only $w$ will be sensitive to only late-time growthgeometry discrepancies, and are more similar to what we study. In this split- $w$-only test, Refs. [40,42] find consistency with $w$ CDM. When Ref. [42] splits both $\Omega_{m}$ and $w$, they find $w^{\text {grow }}>w^{\text {geo }}$ at $3.5 \sigma$, in agreement with a similar 
analysis in Ref. [41], indicating less structure seen by growth observables than geometric ones. However, because of the differences in the analyses, datasets, and treatment of the systematics, we caution against overinterpretation of that comparison.

\section{E. Outlook}

The increasing precision of cosmological measurements will provide opportunities to perform more stringent tests of our standard cosmological model, including via future iterations of growth-geometry split analyses like the one presented here. In the coming months, updated Y3 galaxy clustering and weak lensing measurements will be released which have roughly 3 times the sky area, greater depth, and advances in methodology compared to Y1. Those measurements will provide improved constraints on both cosmological parameters and, crucially for testing growth-geometry consistency, the properties of intrinsic alignments.

It is worth noting that the external likelihoods in this paper were chosen to follow versions used in other DES Y1 papers [30]; updated versions of both the Planck and BOSS likelihoods are already available and could be easily applied to near-future growth-geometry split studies. Relative to Planck 2015, the Planck 2018 cosmology results [57] have slight shifts in several parameters that would affect our compressed likelihood. Of these, the most impactful is that Planck 2018's improved polarization measurements lead to constraints on $A_{\mathrm{s}}$ which shift to lower values as they narrow by about a factor of 2. As we saw in Fig. 7, even a small change in Planck's $A_{\mathrm{s}}$ constraints can have a significant impact on the region of overlap between CMB and latetime growth measurements in the $\sum m_{\nu}-\sigma_{8}$ plane. Lowering and tightening Planck's $A_{\mathrm{s}}$ constraint may be enough to shift Ext-all towards favoring low rather than high values of neutrino mass. This would in turn likely lower the values of $\Omega_{m}^{\text {grow }}$ preferred by both DES + Ext-geo and DES + Ext-all.

One could also consider updating our BAO and RSD measurements to use the recently released eBOSS DR16 [61] measurements, which combine the DR12 BAO galaxies we use with the low- $z$ Main Galaxy Sample (MGS) sample, high- $z$ eBOSS galaxies (LRG and ELG), high $-z$ quasars, and Lyman- $\alpha$ forest mesurements. The high- $z$ BAO measurements tend to prefer lower values of $\Omega_{m}$ and $h$ than the galaxy samples used in DR12, so it is likely that switching to eBOSS would pull our constraints to slightly lower $\Omega_{m}^{\text {geo }}$. However, as our analysis is currently more limited by its ability to constrain growth rather than geometry, the largest impact of switching from BOSS DR12 to eBOSS would be from the inclusion of additional RSD measurements from the MGS, ELG, LRG, and QSO samples. Nearly all of these added samples have $f \sigma_{8}$ constraints that are high relative to the prediction from the Planck 2018 best-fit cosmology, so updating to eBOSS RSD would likely raise the $\sigma_{8}$ value preferred by Ext-all. Referencing Figs. 6 and 7, we predict that this would likely pull our DES + Ext-all results to lower $\Omega_{m}^{\text {grow }}$ by making the Ext-all posterior more compatible with small neutrino mass.

Beyond increasing the precision of individual measurements, including additional, complementary probes of structure growth could benefit future growth-geometry split analyses. We found in this analysis that including growth information from both DES and RSD allowed us to break degeneracies between $\Omega_{m}^{\text {grow }}, \sum m_{\nu}$, and $A_{\mathrm{IA}}$ in order to more robustly test $\Lambda \mathrm{CDM}$. Adding more observables that are sensitive to structure growth can help us further disentangle searches for deviations from $\Lambda \mathrm{CDM}$ from the effects of neutrino mass or astrophysical systematics. One approach to doing this could be to use full-shape information in the galaxy correlations measured by BOSS to directly constrain cosmological parameters, as in Refs. [141-143]. Another would be to include measurements of galaxy clusters. Previous growth-geometry split analyses in Refs. [41,42] report that galaxy cluster number counts significantly influence their growth parameter constraints, though that is complicated by systematics related to the calibration of massobservable relations. Thus, combining galaxy clustering and weak lensing data with galaxy cluster counts, as in Ref. [144], may be a powerful way to break degeneracies with systematics and add constraining power to future tests of growth-geometry consistency. Finally, another promising avenue could be to include CMB lensing data in a combined analysis like those in Refs. [137,145]. Since the CMB lensing kernel reaches higher redshifts than galaxy lensing, this would give us a longer lineof-sight lever arm for probing how LSS has evolved over time.

Looking further ahead, searching for deviations from the predictions of $\Lambda \mathrm{CDM}$, particularly in the evolution of structure growth, will be a core part of future cosmological experiments, including DESI, the Rubin Observatory Legacy Survey of Space and Time, the Nancy Grace Roman Space Telescope, and Euclid, as well as the Simons Observatory and CMB S4. These searches may be conducted in a variety of ways, using parametrizations that range from purely phenomenological splits of data to more physical models derived from modified-gravity actions. Whatever the approach, some findings of this growth-geometry split analysis are broadly applicable: as measurements get more precise, it will only become more important to characterize how searches from beyond$\Lambda \mathrm{CDM}$ physics are influenced by the assumptions about massive neutrinos and astrophysical systematics like intrinsic alignments, and a key way to distinguish between those 
things will be by performing combined analyses of multiple probes of structure growth.

\section{ACKNOWLEDGMENTS}

This paper has gone through internal review by the DES Collaboration. J. M. has been supported by the Porat Fellowship at Stanford University, and by the Rackham Graduate School through a Predoctoral Fellowship. The analysis made use of the software tools SciPy [146], NumPy [147], MATPLOTLIB [148], CAMB [78,79], GetDist [126], MultiNest [122-124], Polychord [125], ANESTHETIC [129], CosmoSis [121], and CosmoLike [98]. It was supported through computational resources and services provided by the National Energy Research Scientific Computing Center (NERSC), a U.S. Department of Energy Office of Science User Facility operated under Contract No. DE-AC02-05CH11231, and by the Sherlock cluster, supported by Stanford University and the Stanford Research Computing Center. Funding for the DES Projects has been provided by the U.S. Department of Energy, the U.S. National Science Foundation, the Ministry of Science and Education of Spain, the Science and Technology Facilities Council of the United Kingdom, the Higher Education Funding Council for England, the National Center for Supercomputing Applications at the University of Illinois at Urbana-Champaign, the Kavli Institute of Cosmological Physics at the University of Chicago, the Center for Cosmology and Astro-Particle Physics at the Ohio State University, the Mitchell Institute for Fundamental Physics and Astronomy at Texas A\&M University, Financiadora de Estudos e Projetos, Fundação Carlos Chagas Filho de Amparo à Pesquisa do Estado do Rio de Janeiro, Conselho Nacional de Desenvolvimento Científico e Tecnológico and the Ministério da Ciência, Tecnologia e Inovação, the Deutsche Forschungsgemeinschaft, and the Collaborating Institutions in the Dark Energy Survey. The Collaborating Institutions are Argonne National Laboratory, the University of California at Santa Cruz, the University of Cambridge, Centro de Investigaciones Energéticas, Medioambientales y Tecnológicas-Madrid, the University of Chicago, University College London, the DES-Brazil Consortium, the University of Edinburgh, the Eidgenössische Technische Hochschule (ETH) Zürich, Fermi National Accelerator Laboratory, the University of Illinois at Urbana-Champaign, the Institut de Ciències de l'Espai (IEEC/CSIC), the Institut de Física d'Altes Energies, Lawrence Berkeley National Laboratory, the LudwigMaximilians Universität München and the associated Excellence Cluster Universe, the University of Michigan, NFS's NOIRLab, the University of Nottingham, The Ohio State University, the University of Pennsylvania, the University of Portsmouth, SLAC National Accelerator Laboratory, Stanford University, the University of Sussex,
Texas A\&M University, and the OzDES Membership Consortium. Based in part on observations at Cerro Tololo Inter-American Observatory at NSF's NOIRLab (NOIRLab Prop. ID 2012B-0001; PI: J. Frieman), which is managed by the Association of Universities for Research in Astronomy (AURA) under a cooperative agreement with the National Science Foundation. The DES data management system is supported by the National Science Foundation under Grants No. AST-1138766 and No. AST-1536171. The DES participants from Spanish institutions are partially supported by MICINN under Grants No. ESP2017-89838, No. PGC2018-094773, No. PGC2018-102021, No. SEV-2016-0588, No. SEV2016-0597, and No. MDM-2015-0509, some of which include ERDF funds from the European Union. I. F. A.E. is partially funded by the CERCA program of the Generalitat de Catalunya. Research leading to these results has received funding from the European Research Council under the European Union's Seventh Framework Program (FP7/20072013) including ERC grant agreements No. 240672, No. 291329, and No. 306478. We acknowledge support from the Brazilian Instituto Nacional de Ciência e Tecnologia (INCT) do e-Universo (CNPq Grant No. 465376/2014-2). This manuscript has been authored by the Fermi Research Alliance, LLC under Contract No. DE-AC02-07CH11359 with the U.S. Department of Energy, Office of Science, Office of High Energy Physics.

\section{APPENDIX A: THE IMPACT OF CHANGING $z_{i}$}

Here we characterize how our expected constraints depend on $z_{i}$, the redshift from Eq. (1) below which the evolution of the linear matter power spectrum is controlled by growth parameters. In addition to our fiducial choice of $z_{i}=3.5$, we analyze the fiducial synthetic data vector with versions of our pipeline that have $z_{i}=5$ and $z_{i}=10$.

Figure 9 shows the impact of these changes. For DES data alone we find that increasing $z_{i}$ from 3.5 to 5 or 10 does slightly affect the upper bounds on $\Omega_{m}^{\text {grow }}$, but it does not significantly impact constraints on $\Omega_{m}^{\text {geo }}$. In this simulated analysis we find that $z_{i}=5$ results in the weakest $\Omega_{m}^{\text {grow }}$ upper bound, while the bound from $z_{i}=10$ is similar to but slightly weaker than the fiducial $z_{i}=3.5$ result. The reason for the lack of monotonic trend in this is not clear, but given DES-only's poor constraining power on growth parameters and its sensitivity to projection effects and systematics (to be discussed in subsequent appendixes), we refrain from overinterpreting this. It is also possible that some of this variation is due to noise in the posterior estimate from Multinest, which can occur because of the small number of samples the posterior tails. Results from the joint analysis of DES + Ext-all are not significantly affected by changing $z_{i}$. 

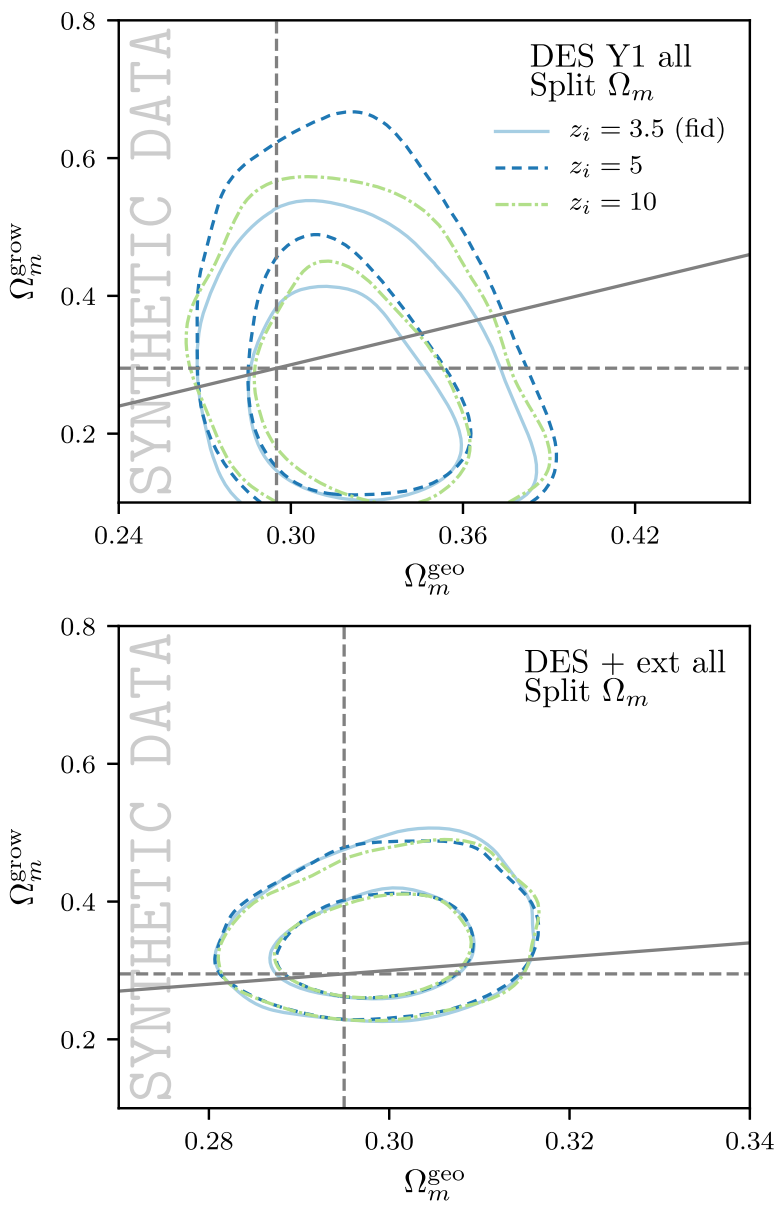

FIG. 9. The impact of changing the redshift $z_{i}$, where growth parameters start controlling the evolution of the matter power spectrum, defined in Eq. (1), for DES-only (top panel) and DES + Ext-all (bottom panel). Countours show the 68\% and $95 \%$ confidence regions from the analysis of synthetic data.

\section{APPENDIX B: EVALUATING PROJECTION EFFECTS}

This appendix discusses insights gained by studying the results of a simulated analysis in which we applied our parameter estimation pipeline to noiseless synthetic data generated by setting observables (e.g., $\mathrm{BAO} \alpha$ parameters, weak lensing $2 \mathrm{PCF}$ ) equal to theory predictions at fiducial parameter values. Specifically, here we focus on the case where the synthetic data is generated using our baseline pipeline - that is, using the same modeling choices as in the theory predictions that we employ for parameter estimation. Before analyzing real data, we use these simulated analyses to identify data and model combinations for which we expect marginalized posteriors to be reliably informative about whether growth and geometry constraints are consistent. This is not guaranteed: in high-dimensional parameter spaces like the one we consider, projecting the posterior volume onto one- or two-dimensional subspaces can result in offsets between the peaks of marginalized posteriors and the best-fit parameter values. By comparing marginalized posteriors from simulated analyses to the known input values, we are able to characterize the extent of these projection effects on our split parameters. We use this comparison to identify which data combinations will the focus of our analysis.

Our main results will be derived from the model and data combinations whose marginalized posteriors in simulated analyses are consistent with the input parameter values. These are as follows:

(1) Split $\Omega_{m}$ constrained with DES + Ext-geo.

(2) Split $\Omega_{m}$ constrained with DES + Ext-all.

For both of these model-data combinations, the input parameter values are contained within the $68 \%$ confidence contour for the synthetic-data version of all twodimensional constraint plots appearing in this paper. The input values also are within the $68 \%$ confidence interval of the one-dimensional marginalized posterior of the split parameter, as well as their differences.

We also consider two additional sets of constraints:

(1) Split $\Omega_{m}$ constrained by DES only.

(2) Split $\Omega_{m}$ and $w$ constrained by DES + Ext-all.

For these we find offsets between the input parameter values and the peaks of the marginalized 1D posteriors of the split parameters. In our simulated analysis, the DESonly marginalized posteriors for $\Omega_{m}^{\text {geo }}$ and $\Omega_{m}^{\text {grow }}$ are biased (high and low, respectively) relative to their input values by about $1 \sigma$. The DES + Ext-all constraints on split $\Omega_{m}$ and $w$ exhibit $\sim 1 \sigma$ offsets for marginalized posteriors of $\Omega_{m}^{\text {grow }}$ and $w^{\text {grow }}$. We therefore treat the results from these constraints with caution. Because we do not trust the onedimensional posterior peaks to accurately reflect the best-fit values, we will not quote their one-dimensional marginalized parameter constraints. However, we will still report model-comparison measures and will show constraint contours for two-dimensional marginalized posteriors. This is motivated by the fact that in our simulated analyses the $68 \%$ confidence intervals of these twodimensional marginalized posteriors do contain the input parameter values. Since simulated analysis for DES + Ext-all, our most constraining dataset, results in constraints on split $\Omega_{m}$ and $w$ that are offset from their input values, we do not consider constraints on the split $w$ model from the less constraining data combinations, DES-only and DES + Ext-geo.

It can be instructive to examine the parameter degeneracies that drive the projection effects described above. The fact that the DES-only constraints on split $\Omega_{m}$ are biased high for $\Omega_{m}^{\text {geo }}$ and low for $\Omega_{m}^{\text {grow }}$ can be understood in terms of a degeneracy between $\Omega_{m}^{\text {grow }}$ and $\Omega_{m}^{\text {geo }}$, as well as degeneracies $\Omega_{m}^{\text {grow }}$ has with the primordial power amplitude $A_{\mathrm{s}}$ and with the intrinsic alignment amplitude $A_{\mathrm{IA}}$. These degeneracies are illustrated (for real data) in Fig. 10. Focusing initially on the DES-only constraints, we note that very low $\Omega_{m}^{\text {grow }}$ values are allowed because they can be 


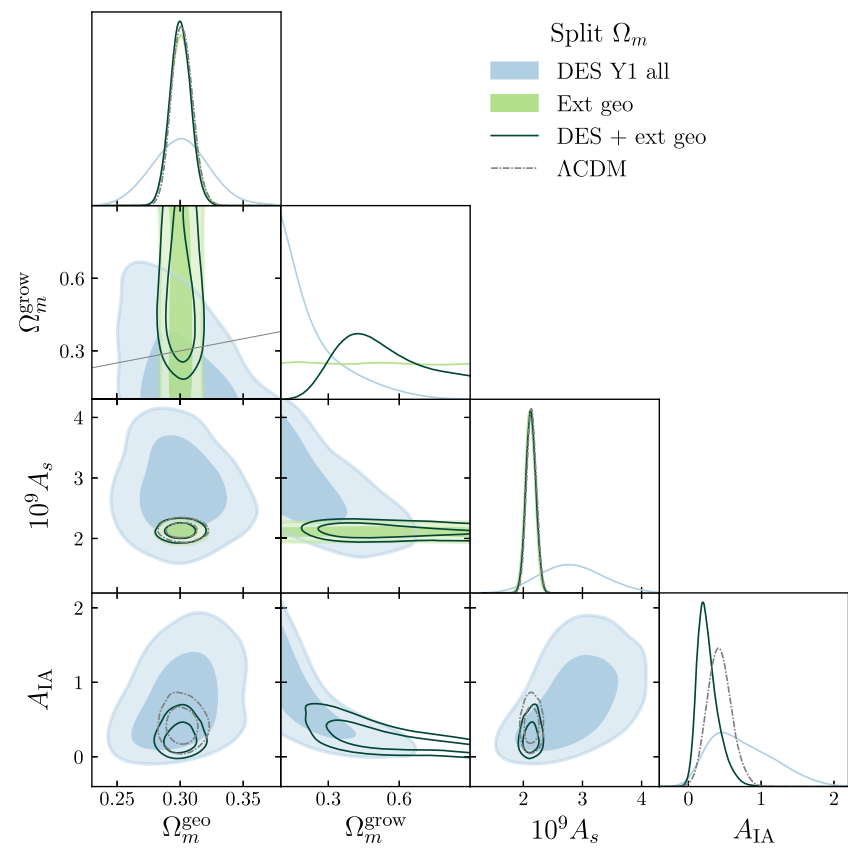

FIG. 10. Constraints illustrating the parameter degeneracies that are relevant to understanding the parameter space projection effects impacting the DES-only constraints on split $\Omega_{m}$. Offdiagonal panels show $68 \%$ and $95 \%$ confidence intervals, with DES-only results in blue, Ext-geo in light green, and DES + Ext-geo as the dark green unshaded contours. The gray diagonal line shows where $\Omega_{m}^{\text {grow }}=\Omega_{m}^{\text {geo }}$, and gray dashed contours show $\Lambda \mathrm{CDM}$ results for DES + Ext-geo.

compensated by raising $A_{\mathrm{s}}$, while very high $\Omega_{m}^{\text {grow }}$ values would presumably be ruled out based on the rate of structure growth occurring over the redshift range probed by DES. The degeneracy with $A_{\mathrm{IA}}$ occurs because intrinsic alignment contributions to the shear 2PCF appear in Eq. (6) via a factor $\propto A_{\mathrm{IA}} \Omega_{m}^{\text {grow }}$. This causes the constant-posterior contours to have a banana shape in the $\Omega_{m}^{\text {grow }}-A_{\text {IA }}$ plane, such that small values of $\Omega_{m}^{\text {grow }}$ allow large values of $A_{\text {IA }}$ and vice versa. These degeneracies combine with the fact that the DES-only likelihood is relatively flat in $\Omega_{m}^{\text {grow }}$ (as can be seen in the profile likelihood shown in Fig. 16 below), to produce an offset in the projected posterior. This translates into an offset in $\Omega_{m}^{\text {geo }}$ as well, because there is a weak degeneracy between $\Omega_{m}^{\text {grow }}$ and $\Omega_{m}^{\text {geo }}$. The DES + Ext-geo and DES + Ext-all constraints do not show these offsets, because the Planck constraints break the $\Omega_{m}^{\text {grow }}-A_{\mathrm{s}}$ degeneracy.

The projection effects for DES + Ext-all constraints on split $\Omega_{m}$ and $w$ are driven by the fact that the effects of $\Omega_{m}^{\text {grow }}$ and $w^{\text {grow }}$ on observables are very degenerate with one another (see Fig. 4). Though each of these growth parameters would have unbiased marginalized constraints if the other were fixed to its fiducial value, they are unconstrained when varied simultaneously. In other words, while the data we consider can constrain deviations from standard structure growth, they are not informative enough to distinguish between $\Omega_{m}$-like and $w$-like changes.

\section{APPENDIX C: IMPACT OF UNMODELED SYSTEMATICS}

We additionally analyze synthetic data where the DES $3 \times 2$ pt measurements are contaminated by unmodeled systematic effects in order to characterize our robustness against certain modeling assumptions. For example, we compute the $3 \times 2$ pt observables using a nonlinear galaxy bias model. By treating those synthetic observables as if they are data and fitting with our fiducial model (which assumes linear galaxy bias), we can quantify the extent to which unmodeled effects (here, the presence nonlinear galaxy bias) biases our cosmological results. The synthetic data vectors we use in this study are the same as those used for similar tests in Refs. [30,98]. They are as follows:

(1) Baseline.-This data vector is equal to a theory prediction at a fiducial $\Lambda \mathrm{CDM}$ cosmology, using the same modeling choices as parameter estimation.

(2) Baryons. - This data vector includes one case of the possible effects of baryonic physics; the impact of AGN feedback on the nonlinear power spectrum is included using the OWLS AGN hydrodynamical simulation [149], following the method described in Ref. [96].

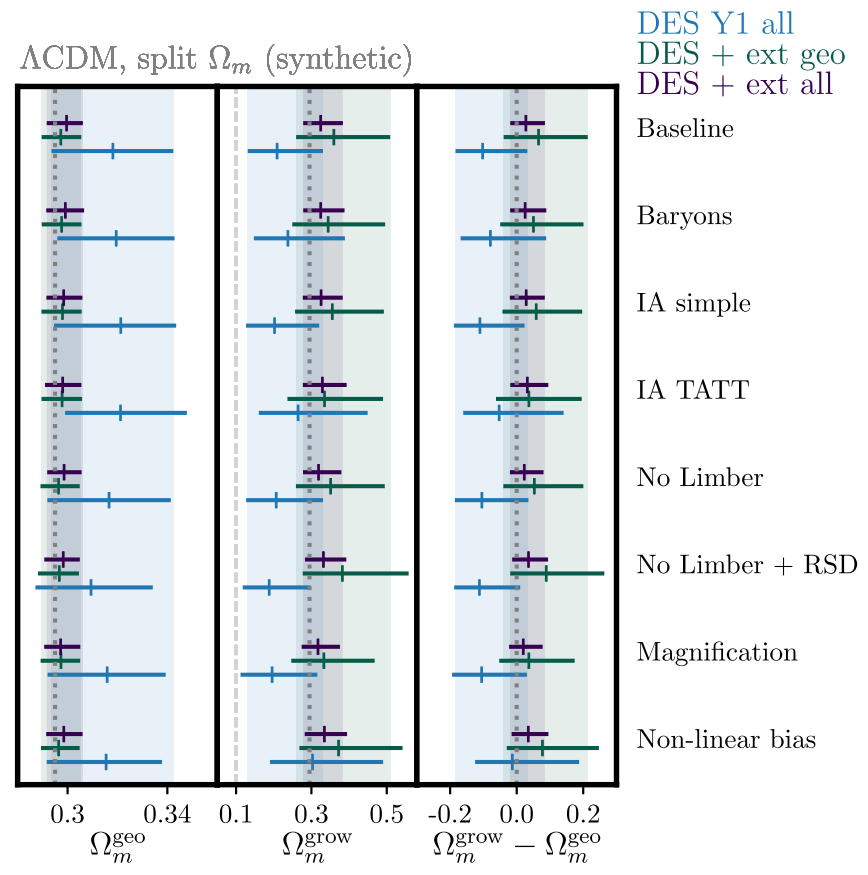

FIG. 11. Robustness of constraints to adding systematics to simulated data, for the split- $\Omega_{m}$ model. Data points and error bars represent the peak and $68 \%$ confidence intervals for marginalized one-dimensional posteriors. The vertical shaded regions correspond to the baseline error bars. 
(3) IA simple.-Using the same nonlinear alignment model as in our fiducial model, this data vector is generated with $A_{\mathrm{IA}}=0.5$ and $\eta_{I A}=0.5$. We note that these parameters are marginalized over in our analysis, so including this data in our tests checks whether degeneracies between the intrinsic alignment parameters and the cosmological parameters can introduce biases.

(4) IA TATT.-Here, the data vector is simulated with a different intrinsic alignment power spectrum shape. It is modeled by assuming all intrinsic alignments are generated by tidal torquing, which is quadratic in the tidal field, instead of the linear tidal alignments described in our fiducial model. To compute it, we use the tidal alignment and tidal torquing model (TATT) [150] with tidal alignment amplitude $A_{1}=0$, tidal torquing amplitude $A_{2}=2$, and no $z$ dependence.

(5) No Limber.-This data vector has been simulated using a theory calculation done without the Limber approximation for $w(\theta)$.

(6) No Limber + RSD.-This data vector has been simulated using a theory calculation done without the Limber approximation and including the contributions of redshift space distortions for $w(\theta)$ as described in Ref. [151].

(7) Magnification.-This data vector is simulated including contributions from magnification to $\gamma_{t}$ and $w(\theta)$, which are added in Fourier space as is described in Ref. [152].

(8) Nonlinear bias.- This data vector goes beyond our fiducial model of linear galaxy bias and models the relationship between matter $\delta$ and galaxy density fluctuations $\delta_{g}$ as $[153,154]$

$$
\delta_{g}=b_{1}^{i} \delta+\frac{1}{2} b_{2}^{i}\left[\delta^{2}-\sigma^{2}\right]
$$

where $\sigma$ is the variance in $\delta$, and $i$ refers to the lens redshift bin. This theory data vector was computed using the FAST-PT code [155] with input values $b^{i}=\{1.45,1.55,1.65,1.8,2.0\}$, and the $b_{2}$ values used are estimated from fits to the Buzzard simulations [156] to be $b_{2}=0.412-2.143 b_{1}+0.929 b_{1}^{2}+$ $0.008 b_{1}^{3}$.

More detailed descriptions of the generation of these data can be found in Refs. [30,98].
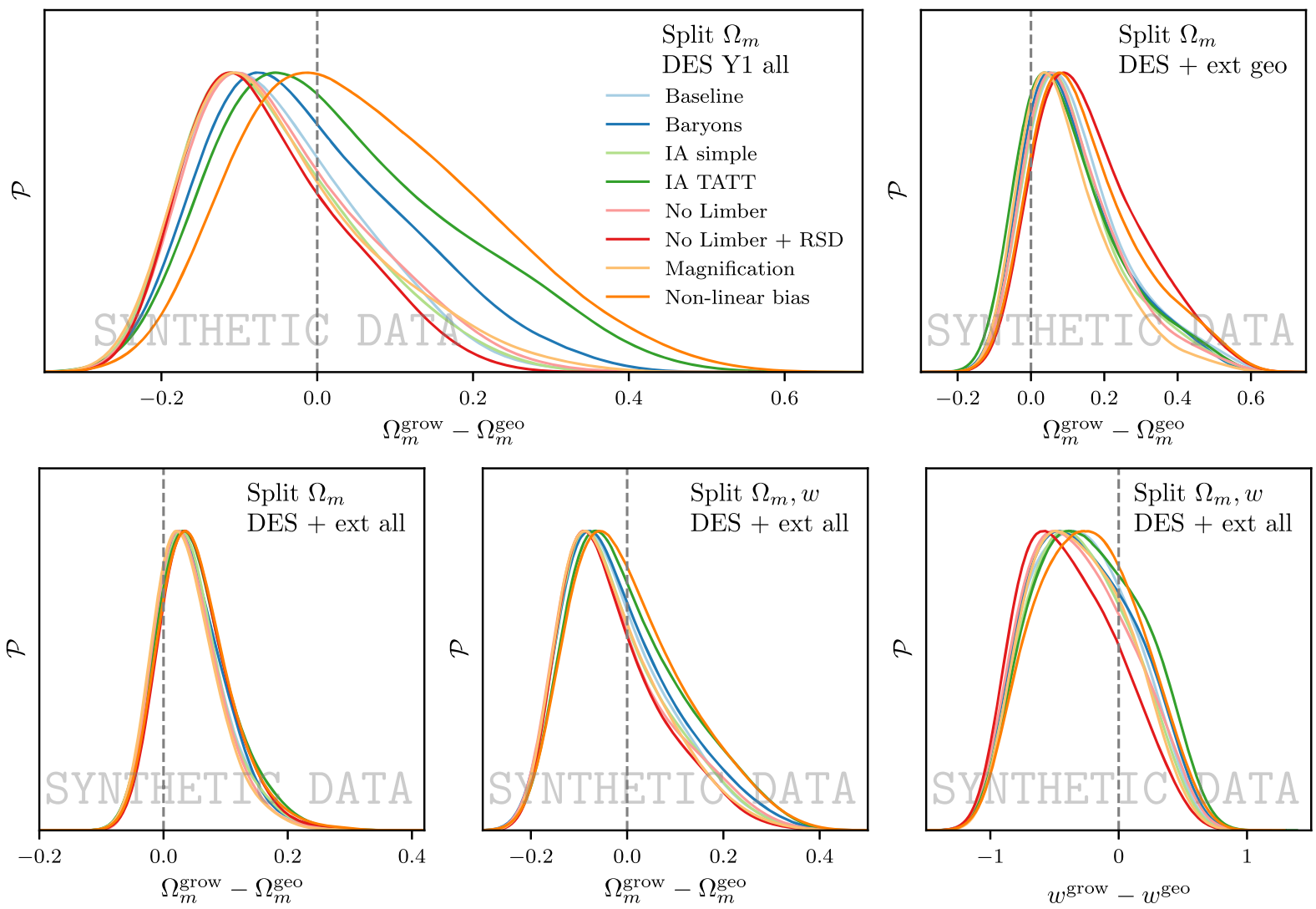

FIG. 12. Marginalized posteriors for synthetic data vectors contaminated by systematic effects, showing constraints on the difference between growth and geometry parameters. Top row: Split- $\Omega_{m}$ results for DES-only (left) and for DES + Ext-geo (right). Bottom row: Results from DES + Ext-all when we split $\Omega_{m}$ (left), and when we split both $\Omega_{m}$ and $w$, showing growth-geometry differences for $\Omega_{m}$ (center) and $w$ (right). 
The metric for passing these tests is based on the onedimensional marginalized posteriors for $\Omega_{m}^{\text {grow }}$ and $\Omega_{m}^{\text {geo }}$, as well as the $w^{\text {grow }}$ and $w^{\text {geo }}$ for the parametrization where $w$ is split. For each of the data combinations discussed above, we verify that the shift in the peak of the posterior is less than $0.3 \sigma$ relative to the baseline analysis. We evaluate the size of these shifts by computing an effective $\sigma$ by summing the two posteriors' asymmetric $68 \%$ confidence intervals in quadrature. To state this more specifically, let $\hat{\theta}$ be the onedimensional marginalized posterior peak on parameter $\theta$, and suppose the baseline and contaminated constraints are labeled $A$ and $B$ such that $\hat{\theta}_{A}>\hat{\theta}_{B}$. Additionally, let $\theta_{A}^{\text {low68 }}$ be the lower bound of the $68 \%$ confidence interval for dataset $A$ and let $\theta_{B}^{\text {up} 68}$ be the upper bound of the $68 \%$ confidence interval for dataset $B$. We quantify the size of the posterior shift as

$$
\Delta_{\theta}=\frac{\hat{\theta}_{A}-\hat{\theta}_{B}}{\sqrt{\left(\hat{\theta}_{a}-\theta_{A}^{\text {low68 }}\right)^{2}+\left(\theta_{B}^{\text {up } 68}-\hat{\theta}_{B}\right)^{2}}} .
$$

Summary plots showing the results for these tests are shown in Fig. 11 for split $\Omega_{m}$ and in Fig. 14 for split $\Omega_{m}$ and $w$. We additionally show in Fig. 12 how the posteriors from these same synthetic data analyses project onto the onedimensional marginalized posteriors of the differences $\Omega_{m}^{\text {grow }}-\Omega_{m}^{\text {geo }}$ and $w^{\text {grow }}-w^{\text {geo }}$.

Both of the main model and data combinations identified in our fiducial simulated analysis of Appendix B (DES + Ext-geo and DES + Ext-all constraints on split $\Omega_{m}$ ) pass these tests, as none of these changes results in a parameter shift larger than $0.3 \sigma$. For split $\Omega_{m}$ DES + Ext-geo results, the largest posterior shift observed is in $\Omega_{m}^{\text {grow }}$ and occurs when we add the effects of magnification to the synthetic data. The size of this shift is $-0.16 \sigma$ relative to the baseline simulated analysis. For DES + Ext-all, the systematic with the largest impact is nonlinear galaxy bias, which shifts the $\Omega_{m}^{\text {grow }}$ posterior by $+0.13 \sigma$.

In addition to the prior volume effects described in Appendix B, the DES-only split $\Omega_{m}$ constraints and the DES + Ext-all constraints on split $\Omega_{m}$ and $w$ should be treated with caution because they fail these tests, in the sense that the some of the systematics produce parameter shifts larger than our desired $0.3 \sigma$ threshold. For the DES-only split- $\Omega_{m}$ results, this occurs for TATT intrinsic alignments, which changes the best-fit $\Omega_{m}^{\text {grow }}$ by $+0.34 \sigma$, and for nonlinear bias, which changes $\Omega_{m}^{\text {grow }}$ by $+0.51 \sigma$. All other shifts are below $0.3 \sigma$. For the DES + Ext-all constraints on split $\Omega_{m}$ and $w$, the only systematic that generates a parameter shift larger than our threshold is the non-Limber and RSD modeling for galaxy clustering, which changes $w^{\text {grow }}$ by $-0.36 \sigma$.

\section{APPENDIX D: IMPACT OF CHANGING ANALYSIS CHOICES}

Before revealing the nonoffset (unblinded) parameter estimates, we test the robustness of our analysis of real data against changes to various analysis choices. We perform this test similarly to the systematics tests described in Appendix C, but instead of analyzing contaminated synthetic data, we compare parameter estimates obtained by running on the same real data, but altering aspects of our analysis pipeline. These changes are, following the analysis in Ref. [30],

(1) Free IA z-evolution.- - Instead of assuming that the intrinsic alignment amplitude scales as a power law in redshift, we allow its amplitude to vary for independently for each source redshift bin.

(2) Conservative scale cuts.-We restrict our analysis to DES 2PCF measurements to large angles which are insensitive to nonlinear LSS modeling.

(3) COSMOS photo-z's.-We use alternative photometric redshifts for the DES source galaxies, obtained by resampling COSMOS data following the procedure in Ref. [93].

Additionally, for the split $\Omega_{m}$ model we also show the results of additional tests examining the impact of changing the treatment of the Hubble parameter in our split parametrization. Recalling that our fiducial analysis splits $h$ and fixes $h^{\text {grow }}=0.6881$, we show how parameter constraints change for

(1) Varying $h^{\text {grow }}$. -We allow $h^{\text {grow }}$ to vary over the same [0.1,0.9] prior range as $h \equiv h^{\text {geo }}$.

(2) $h$ not split.-We require $h^{\text {grow }}=h^{\text {geo }}$ and vary it as in $\Lambda \mathrm{CDM}$.

These $h$ tests were conducted after the true analysis results were revealed (after unblinding).

The results of these tests are summarized in Fig. 13 for the split $\Omega_{m}$ analysis, and in Fig. 15 for split $\Omega_{m}$ and $w$. We quantify the changes from the baseline analysis following the same method as in Appendix $\mathrm{C}$ above.

Notably, for all data and model combinations, we see significant parameter shifts in growth parameter estimates when we allow the intrinsic alignment amplitude to vary independently in each redshift bin. For all pipeline variations other than free IA $z$-evolution, we find that our main results, DES + Ext-geo and DES + Ext-all constraints on split $\Omega_{m}$, are robust. For DES + Ext-geo, the largest parameter shift relative to the baseline analysis is a $+0.25 \sigma$ change in $\Omega_{m}^{\text {geo }}$, which occurs when we switch to conservative scale cuts. For DES + Ext-all, the conservative scale cuts and not splitting $h$ tie for the largest shift, a $-0.10 \sigma$ change in $\Omega_{m}^{\text {grow }}$.

The DES-only split $\Omega_{m}$ results and the DES + Ext-all results for split $\Omega_{m}$ and $w$ are less robust, even setting the free IA $z$-evolution results aside. For the DES-only constraints on split $\Omega_{m}$, conservative scale cuts produce a $0.38 \sigma$ shift in $\Omega_{m}^{\mathrm{geo}}$, while all other parameter shifts are 


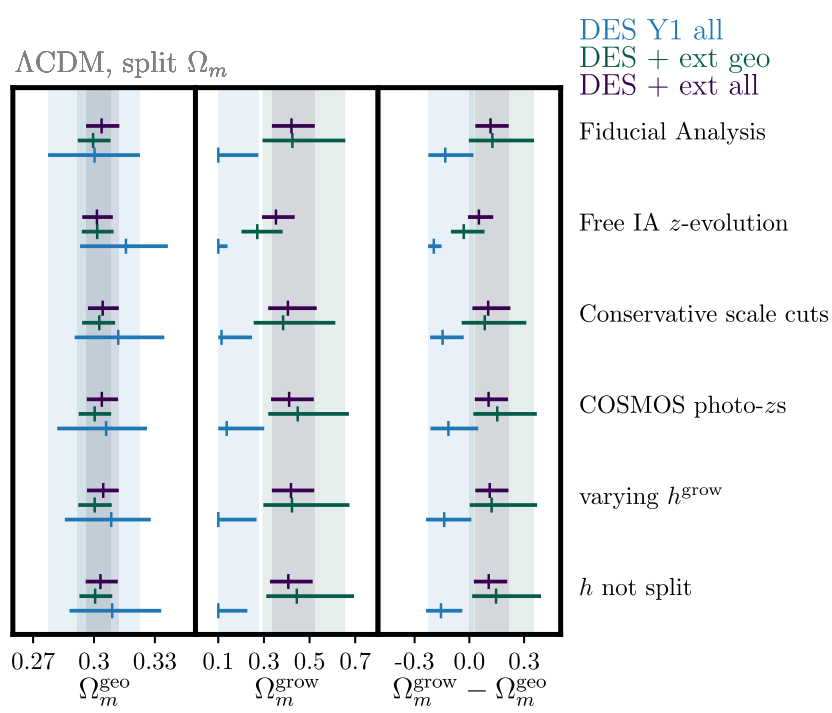

FIG. 13. Robustness of real-data constraints to changes in analysis choices when we split $\Omega_{m}$. Data points and error bars represent the peak and $68 \%$ confidence intervals for marginalized one-dimensional posteriors. The vertical shaded regions correspond to the $68 \%$ confidence interval of the baseline measurements.

below $0.3 \sigma$. For DES + Ext-all constraints on split $\Omega_{m}$ and $w$, using conservative scale cuts moves $\Omega_{m}^{\text {grow }}$ by a $0.31 \sigma$ shift and $w^{\text {grow }}$ by $+0.59 \sigma$, and using the COSMOS photo$z$ 's causes $w^{\text {grow }}$ to change by $0.36 \sigma$.

After observing this behavior in parameter-offset (blinded) results from real data, we performed an analysis of synthetic data using the binned-IA model in order to better characterize its impact, and found that free IA $z$ evolution produced a similar change in posteriors. We hypothesize that the large parameter shifts, especially in $\Omega_{m}^{\text {grow }}$, occur when we introduce more freedom in IA redshift evolution because of a parameter-space projection effect. As discussed for our fiducial NLA IA model in Appendix B, the fact that $\Omega_{m}^{\text {grow }}$ is poorly constrained and degenerate with $A_{\mathrm{IA}}$ causes the DES-only posterior to be skewed towards low $\Omega_{m}^{\text {grow }}$ values. When we allow the IA amplitude to vary independently for each source redshift bin, this opens a large volume of parameter space where small $\Omega_{m}^{\text {grow }}$ can compensate large IA amplitudes. That low $-\Omega_{m}^{\text {grow }}$ posterior volume is much larger than the allowed region of parameter space where $\Omega_{m}^{\text {grow }}$ is high but all four IA amplitudes are small. This means that in the absence of strong constraints on $\Omega_{m}^{\text {grow }}$, small $\Omega_{m}^{\text {grow }}$ values will dominate one- or two-dimensional projections of the posterior. Degeneracies between $\Omega_{m}^{\text {grow }}$ and other parameters will propagate that effect to other parameters like $\Omega_{m}^{\text {geo }}$. This is perhaps analogous to the finding of Ref. [136] that opening up "too much" freedom in the IA model causes $S_{8}$ constraints to shift to smaller values, and we posit that this is why opening up additional IA parameter space causes such dramatic parameter shifts in Figs. 13 and 15.

To support this hypothesis, in Fig. 16 we show the profile likelihood for $\Omega_{m}^{\text {grow }}$ for DES-only and DES + Ext-all constraints on synthetic data. The vertical axes of these plots show the maximum likelihood in our chain samples which have $\Omega_{m}^{\text {grow }}$ within a narrow bin. These profiles are noisy and exhibit sharp dropoffs because our sampler
Split $\Omega_{m}, w$ (synthetic)

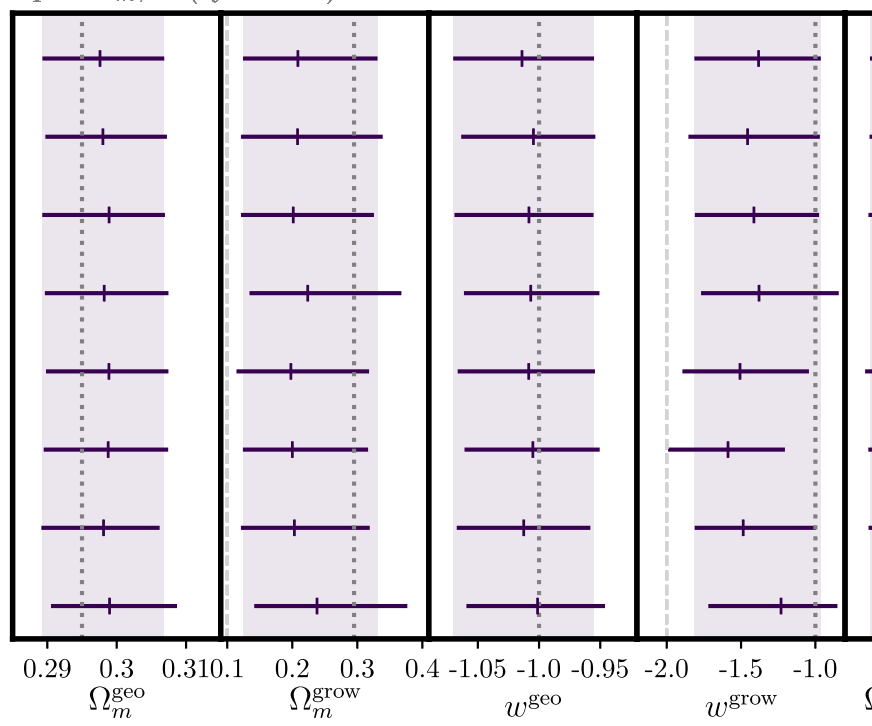

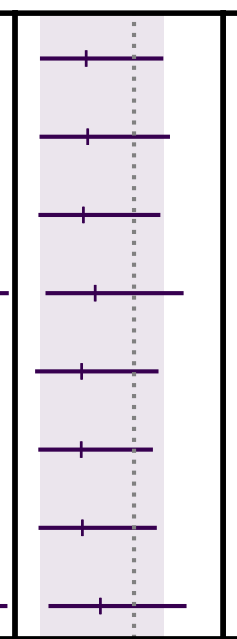

$\begin{array}{lllllll}-0.1 & 0.0 & 0.1 & -0.8 & -0.4 & 0.0\end{array}$ $\Omega_{m}^{\text {grow }}-\Omega_{m}^{\text {geo }} \quad w^{\text {grow }}-w^{\text {geo }}$
DES + ext all

Baseline

Baryons

IA simple

IA TATT

No Limber

No Limber + RSD

Magnification

Non-linear bias

FIG. 14. Robustness of results to adding systematics to simulated data for the model splitting $\Omega_{m}$ and $w$. Data points and error bars represent the peak and $68 \%$ confidence intervals for marginalized one-dimensional posteriors. The vertical shaded regions correspond to the baseline error bars. 


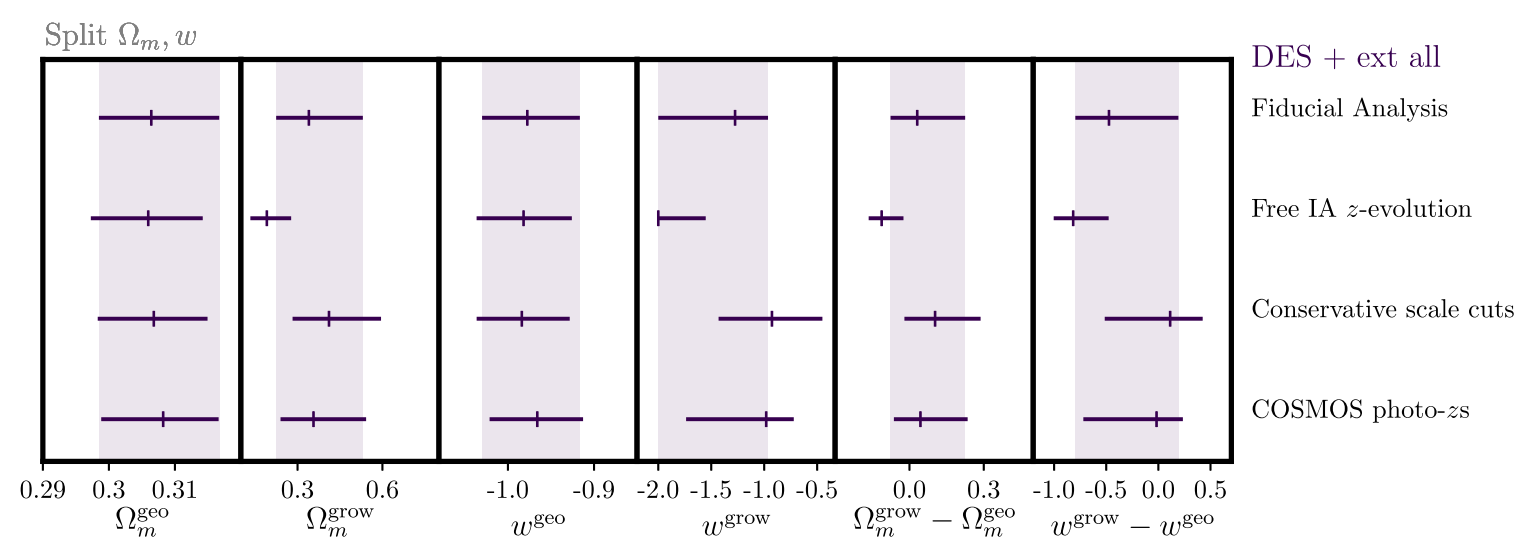

FIG. 15. Robustness of real-data constraints to changes in analysis choices when we split $\Omega_{m}$ and $w$. Data points and error bars represent the peak and $68 \%$ confidence intervals for marginalized one-dimensional posteriors. The vertical shaded regions correspond to the $68 \%$ confidence interval of the baseline measurements.

(Multinest in this case) returned very few chain samples in that region of parameter space. Where there are enough samples to compare the baseline and binned-IA profiles, we see that they have very similar profile likelihoods. This means that the "free IA $z$-evolution" model does not actually produce an improved fit to the data at small $\Omega_{m}$ compared to our fiducial model. Rather, the posterior peaks at smaller $\Omega_{m}^{\text {grow }}$ because binning IA increases the relative
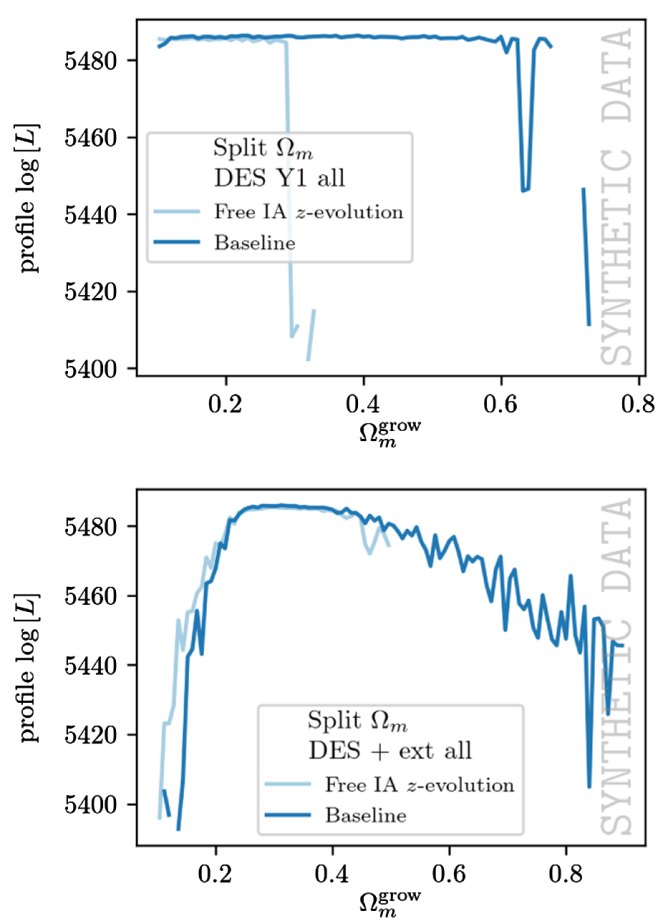

FIG. 16. Likelihood profile showing the maximum likelihood for chain samples within narrow bins of $\Omega_{m}^{\text {grow }}$. The sharp step functions in the DES-only plot show where the sample density decreases significantly due to the $A_{\text {IA }}-\Omega_{m}^{\text {grow }}$ projection effects discussed in Appendix B. volume of the parameter space, and thus the density of chain samples, associated with small $\Omega_{m}^{\text {grow }}$ compared to high $\Omega_{m}^{\text {grow }}$. In other words, the change in posterior peak comes from parameter volume projection effects.

Current data $[3,136,137]$ are not able to rule out models with this much variation in the IA amplitude, but neither do they provide evidence for IA redshift evolution beyond our NLA power law, nor is there a strong theoretical motivation for it. Given this, we proceed with our analysis despite the nominal failure of this robustness test.

\section{APPENDIX E: ADDITIONAL RESULTS}

\section{Parameter degeneracies without RSD data}

We include Fig. 17 to supplement the discussion in Sec. VI about how splitting $\Omega_{m}$ impacts constraints on $\sum m_{\nu}, S_{8}, h$, and $A_{\mathrm{IA}}$. It is identical to Fig. 6, except that it does not include BOSS RSD measurements. The offdiagonal panels show the $68 \%$ and $95 \%$ confidence intervals for DES-only as blue shaded regions (identical to those in Fig. 6), those for Ext-geo in light green shaded regions, and those for the DES + Ext-geo combination as dark green unshaded contours. Diagonal gray lines denote where $\Omega_{m}^{\text {grow }}=\Omega_{m}^{\text {geo }}$, and gray dashed contours show $\Lambda \mathrm{CDM}$ results for DES + Ext-geo. The diagonal panels show normalized marginalized posteriors for individual parameters.

\section{Impact of fixing $\sum \boldsymbol{m}_{\nu}$}

Here we present additional information about the impact of fixing neutrino mass to supplement the discussion and Fig. 8 in Sec. VI A. Table IV reports tension and model comparison metrics for the fixed neutrino mass analyses. It follows the same notation and conventions as what is used for the main results in Table III. 


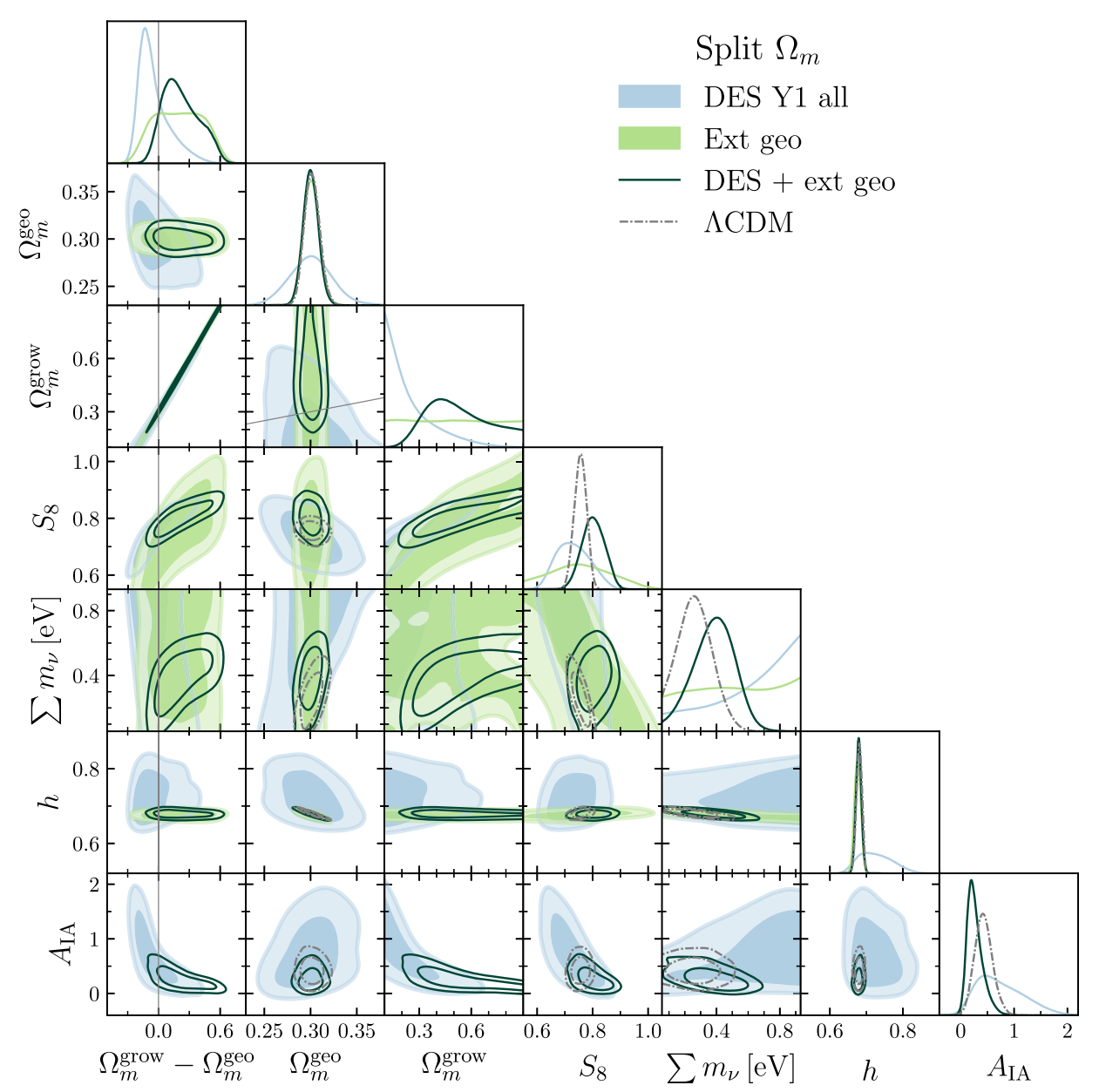

FIG. 17. Marginalized constraints from DES and Ext-geo data. This plot is identical to Fig. 6, but uses the external dataset that does not include BOSS RSD.

TABLE IV. Constraints, tension, and model comparison statistics for split parameters when we fix $\sum m_{\nu}=0.06 \mathrm{eV}$.

\begin{tabular}{lccc}
\hline \hline$\Lambda$ CDM, & & & \\
Fix $m_{\nu}$ & DES & DES + Ext-geo & DES + Ext-all \\
\hline$\Omega_{m}$ & $0.279_{-0.017}^{+0.023}$ & $0.289_{-0.005}^{+0.007}$ & $0.290_{-0.006}^{+0.007}$ \\
$\tilde{d}$ & $13.4 \pm 0.7$ & $15.7 \pm 0.7$ & $16.1 \pm 0.7$ \\
$\log S^{\text {dat }}$ & $\ldots$ & $-0.7 \pm 0.2$ & $-1.1 \pm 0.2$ \\
$p\left(S>S^{\text {dat }}\right)$ & $\ldots$ & $0.23 \pm 0.05$ & $0.15 \pm 0.05$ \\
Equiv. $\sigma$ & $\ldots$ & $1.2 \pm 0.1$ & $1.4 \pm 0.2$ \\
\hline \hline Split $\Omega_{m}$, & & & \\
Fix $m_{\nu}$ & & DES + Ext-geo & \\
\hline$\Omega_{m}^{\text {grow }}-\Omega_{m}^{\text {geo }}$ & DES & $-0.040_{-0.055}^{+0.074}$ & $-0.007_{-0.037}^{+0.036}$ \\
$\Omega_{m}^{\text {geo }}$ & $\ldots$ & $0.291_{-0.007}^{+0.007}$ & $0.292_{-0.006}^{+0.006}$ \\
$\Omega_{m}^{\text {grow }}$ & $\ldots$ & $0.252_{-0.071}^{+0.070}$ & $0.284_{-0.035}^{+0.036}$ \\
$\tilde{d}$ & $\ldots$ & $17.6 \pm 0.9$ & $16.8 \pm 0.8$ \\
$\log S^{\text {dat }}$ & $14.5 \pm 0.7$ & $-1.2 \pm 0.3$ & $-0.5 \pm 0.3$ \\
$p\left(S>S^{\text {dat }}\right)$ & $\ldots$ & $0.11 \pm 0.06$ & $0.29 \pm 0.06$ \\
Equiv. $\sigma$ & $\ldots$ & $1.6 \pm 0.3$ & $1.1 \pm 0.1$ \\
$\log S^{\text {mod }}$ & $\ldots$ & $0.4 \pm 0.2$ & $0.0 \pm 0.2$ \\
$p\left(S>S^{\text {mod }}\right)$ & $0.0 \pm 0.2$ & $0.57 \pm 0.20$ & $0.39 \pm 0.23$ \\
Equiv. $\sigma$ & $0.45 \pm 0.24$ & $0.5 \pm 0.3$ & $0.9 \pm 0.4$ \\
\hline \hline
\end{tabular}




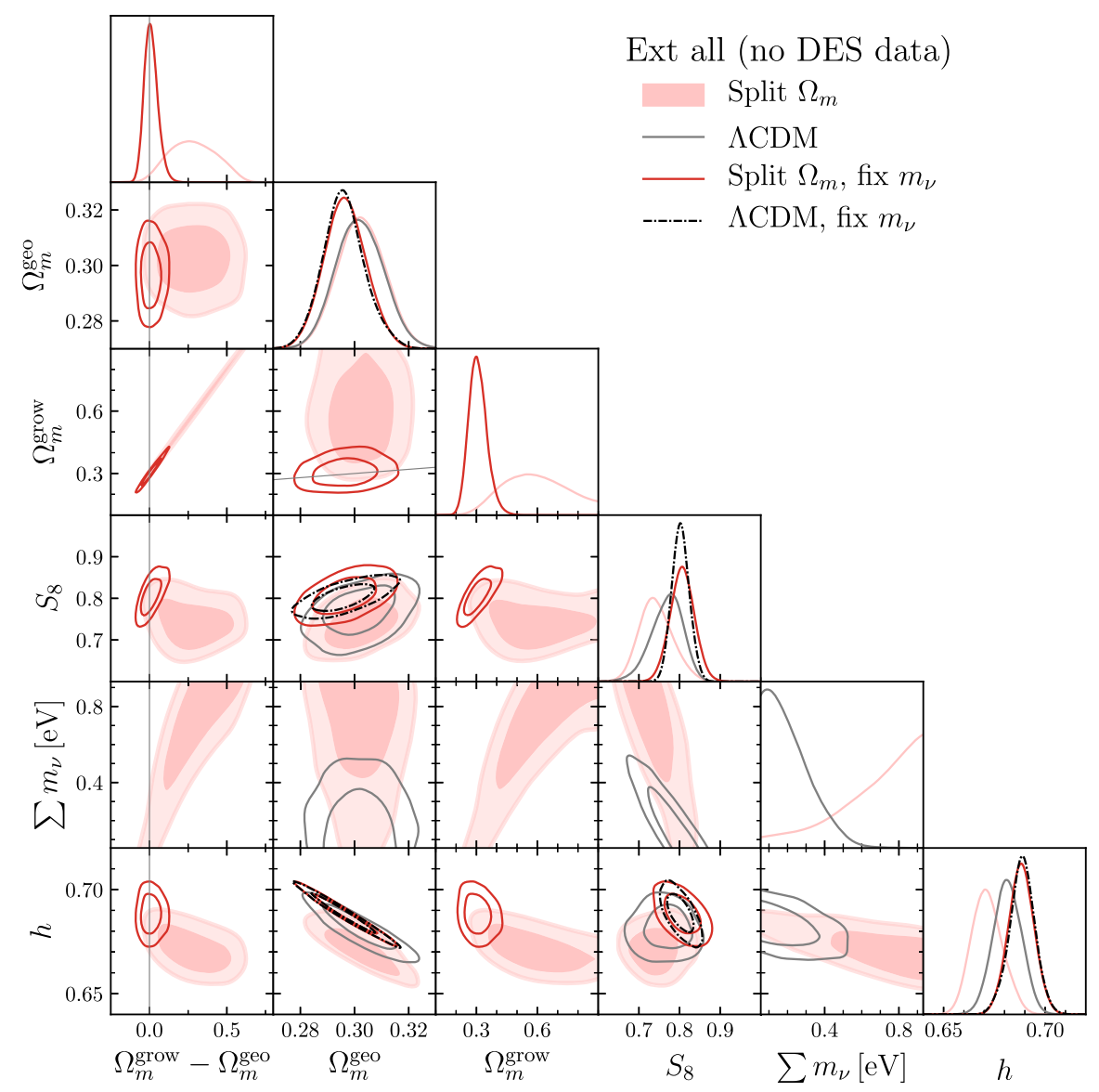

FIG. 18. Ext-all constraints (not including any DES information) showing the effect of fixing neutrino mass for both the split $\Omega_{m}$ parametrization and $\Lambda \mathrm{CDM}$.

Additionally, Fig. 18 shows how the Ext-all constraints change when we either fix $\sum m_{\nu}$ to its minimum allowed value or we revert to $\Lambda \mathrm{CDM}$ with $\Omega_{m}^{\text {grow }}=\Omega_{m}^{\text {geo }}$. In that figure, the shaded pink contours, which are the same as those in Fig. 6, show our baseline DES + Ext-all constraints when $\Omega_{m}$ is split and the sum of neutrino masses is varied. The solid red contours show how these constraints change when we fix $\sum m_{\nu}=0.06 \mathrm{eV}$, while the solid gray contours show what happens when we switch to $\Lambda \mathrm{CDM}$ (but still vary neutrino mass). The dashed black lines show what happens when we both require $\Omega_{m}^{\text {grow }}=\Omega_{m}^{\text {geo }}$ and fix $\sum m_{\nu}$.
[1] A. Joyce, B. Jain, J. Khoury, and M. Trodden, Beyond the cosmological standard model, Phys. Rep. 568, 1 (2015).

[2] D. H. Weinberg, M. J. Mortonson, D. J. Eisenstein, C. Hirata, A. G. Riess, and E. Rozo, Observational probes of cosmic acceleration, Phys. Rep. 530, 87 (2013).

[3] Dark Energy Survey (DES) Collaboration, Dark Energy Survey year 1 results: Cosmological constraints from galaxy clustering and weak lensing, Phys. Rev. D 98, 043526 (2018).

[4] W. Handley and P. Lemos, Quantifying tensions in cosmological parameters: Interpreting the DES evidence ratio, Phys. Rev. D 100, 043504 (2019).
[5] H. Hildebrandt et al., KiDS-450: Cosmological parameter constraints from tomographic weak gravitational lensing, Mon. Not. R. Astron. Soc. 465, 1454 (2017).

[6] S. Joudaki et al., CFHTLenS revisited: assessing concordance with Planck including astrophysical systematics, Mon. Not. R. Astron. Soc. 465, 2033 (2017).

[7] S. Joudaki, M. Kaplinghat, R. Keeley, and D. Kirkby, Model independent inference of the expansion history and implications for the growth of structure, Phys. Rev. D 97, 123501 (2018).

[8] A. Leauthaud et al., Lensing is low: cosmology, galaxy formation or new physics?, Mon. Not. R. Astron. Soc. 467, 3024 (2017). 
[9] E. van Uitert et al., KiDS+GAMA: cosmology constraints from a joint analysis of cosmic shear, galaxy-galaxy lensing, and angular clustering, Mon. Not. R. Astron. Soc. 476, 4662 (2018).

[10] S. Joudaki et al., KiDS-450 + 2dFLenS: Cosmological parameter constraints from weak gravitational lensing tomography and overlapping redshift-space galaxy clustering, Mon. Not. R. Astron. Soc. 474, 4894 (2018).

[11] C. Hikage et al. (HSC Collaboration), Cosmology from cosmic shear power spectra with Subaru Hyper SuprimeCam first-year data, Publ. Astron. Soc. Jpn. 71, 43 (2019).

[12] T. Hamana et al., Cosmological constraints from cosmic shear two-point correlation functions with HSC survey first-year data , Publ. Astron. Soc. Jpn. 72, 16 (2020).

[13] C. Heymans et al. (KiDS Collaboration), KiDS-1000 Cosmology: Multi-probe weak gravitational lensing and spectroscopic galaxy clustering constraints, arXiv:2007 .15632 .

[14] Y. Park and E. Rozo, Concordance cosmology?, Mon. Not. R. Astron. Soc. 499, 4638 (2020).

[15] A. G. Riess et al., A $2.4 \%$ determination of the local value of the hubble constant, Astrophys. J. 826, 56 (2016).

[16] A. G. Riess, S. Casertano, W. Yuan, L. M. Macri, and D. Scolnic, Large magellanic cloud cepheid standards provide a $1 \%$ foundation for the determination of the Hubble constant and stronger evidence for physics beyond $\Lambda \mathrm{CDM}$, Astrophys. J. 876, 85 (2019).

[17] L. Knox and M. Millea, Hubble constant hunter's guide, Phys. Rev. D 101, 043533 (2020).

[18] J. C. Hill, E. McDonough, M. W. Toomey, and S. Alexander, Early dark energy does not restore cosmological concordance, Phys. Rev. D 102, 043507 (2020).

[19] E. Di Valentino, C. Bøehm, E. Hivon, and F. R. Bouchet, Reducing the $H_{0}$ and $\sigma 8$ tensions with dark matterneutrino interactions, Phys. Rev. D 97, 043513 (2018).

[20] S. J. Clark, K. Vattis, and S. M. Koushiappas, CMB constraints on late-universe decaying dark matter as a solution to the $H_{0}$ tension, arXiv:2006.03678.

[21] A. Chen et al. (DES Collaboration), Constraints on Decaying Dark Matter with DES-Y1 and external data, arXiv:2011.04606.

[22] S. Aiola et al. (ACT Collaboration), The Atacama Cosmology Telescope: DR4 Maps and Cosmological Parameters, arXiv:2007.07288.

[23] DES and SPT Collaborations, Dark Energy Survey year 1 results: Joint analysis of galaxy clustering, galaxy lensing, and CMB lensing two-point functions, Phys. Rev. D 100, 023541 (2019).

[24] F. Bianchini et al. (SPT Collaboration), Constraints on Cosmological Parameters from the $500 \mathrm{deg}^{2}$ SPTpol Lensing Power Spectrum, Astrophys. J. 888, 119 (2020).

[25] M. Ishak, A. Upadhye, and D. N. Spergel, Probing cosmic acceleration beyond the equation of state: Distinguishing between dark energy and modified gravity models, Phys. Rev. D 74, 043513 (2006).

[26] E. V. Linder, Cosmic growth history and expansion history, Phys. Rev. D 72, 043529 (2005).

[27] L. Knox, Y.-S. Song, and J. A. Tyson, Distance-redshift and growth-redshift relations as two windows on acceleration and gravitation: Dark energy or new gravity?, Phys. Rev. D 74, 023512 (2006).

[28] E. Bertschinger, On the growth of perturbations as a test of dark energy, Astrophys. J. 648, 797 (2006).

[29] D. Huterer and E. V. Linder, Separating dark physics from physical darkness: Minimalist modified gravity versus dark energy, Phys. Rev. D 75, 023519 (2007).

[30] Dark Energy Survey (DES) Collaboration, Dark Energy Survey year 1 results: Constraints on extended cosmological models from galaxy clustering and weak lensing, Phys. Rev. D 99, 123505 (2019).

[31] Dark Energy Survey (DES) Collaboration, Dark Energy Survey Year 1 results: Measurement of the baryon acoustic oscillation scale in the distribution of galaxies to redshift 1 , Mon. Not. R. Astron. Soc. 483, 4866 (2019).

[32] Dark Energy Survey (DES) Collaboration, Dark Energy Survey Year 1 Results: Cosmological constraints from cluster abundances and weak lensing, Phys. Rev. D 102, 023509 (2020).

[33] Dark Energy Survey (DES) Collaboration, First cosmology results using type ia supernovae from the dark energy survey: constraints on cosmological parameters, Astrophys. J. 872, L30 (2019).

[34] Dark Energy Survey (DES) Collaboration, Cosmological Constraints from Multiple Probes in the Dark Energy Survey, Phys. Rev. Lett. 122, 171301 (2019).

[35] K. N. Abazajian and S. Dodelson, Neutrino Mass and Dark Energy from Weak Lensing, Phys. Rev. Lett. 91, 041301 (2003).

[36] B. Jain and A. Taylor, Cross-Correlation Tomography: Measuring Dark Energy Evolution with Weak Lensing, Phys. Rev. Lett. 91, 141302 (2003).

[37] J. Zhang, L. Hui, and A. Stebbins, Isolating geometry in weak lensing measurements, Astrophys. J. 635, 806 (2005).

[38] F. Simpson and S. Bridle, Illuminating dark energy with cosmic shear, Phys. Rev. D 71, 083501 (2005).

[39] H. Zhan, L. Knox, and J. A. Tyson, Distance, growth factor, and dark energy constraints from photometric baryon acoustic oscillation and weak lensing measurements, Astrophys. J. 690, 923 (2009).

[40] S. Wang, L. Hui, M. May, and Z. Haiman, Is modified gravity required by observations? An empirical consistency test of dark energy models, Phys. Rev. D 76, 063503 (2007).

[41] E. J. Ruiz and D. Huterer, Testing the dark energy consistency with geometry and growth, Phys. Rev. D 91, 063009 (2015).

[42] J. L. Bernal, L. Verde, and A. J. Cuesta, Parameter splitting in dark energy: is dark energy the same in the background and in the cosmic structures?, J. Cosmol. Astropart. Phys. 02 (2016) 059.

[43] W. Lin and M. Ishak, Cosmological discordances: A new measure, marginalization effects, and application to geometry versus growth current data sets, Phys. Rev. D 96, 023532 (2017).

[44] P. Zhang, M. Liguori, R. Bean, and S. Dodelson, Probing Gravity at Cosmological Scales by Measurements which Test the Relationship between Gravitational Lensing and Matter Overdensity, Phys. Rev. Lett. 99, 141302 (2007). 
[45] L. Amendola, M. Kunz, M. Motta, I. D. Saltas, and I. Sawicki, Observables and unobservables in dark energy cosmologies, Phys. Rev. D 87, 023501 (2013).

[46] A. Abate and O. Lahav, The three faces of $\Omega_{m}$ : testing gravity with low- and high-redshift $\mathrm{SNe}$ Ia surveys, Mon. Not. R. Astron. Soc. 389, L47 (2008).

[47] M. Chu and L. Knox, Testing cosmological models and understanding cosmological parameter determinations with metaparameters, Astrophys. J. 620, 1 (2005).

[48] J. M. Z. Matilla, Z. Haiman, A. Petri, and T. Namikawa, Geometry and growth contributions to cosmic shear observables, Phys. Rev. D 96, 023513 (2017).

[49] S. Alam et al. (BOSS Collaboration), The clustering of galaxies in the completed SDSS-III Baryon Oscillation Spectroscopic Survey: cosmological analysis of the DR12 galaxy sample, Mon. Not. R. Astron. Soc. 470, 2617 (2017).

[50] S. Basilakos and F. K. Anagnostopoulos, Growth index of matter perturbations in the light of Dark Energy Survey, Eur. Phys. J. C 80, 212 (2020).

[51] A. Ferté, D. Kirk, A. R. Liddle, and J. Zuntz, Testing gravity on cosmological scales with cosmic shear, cosmic microwave background anisotropies, and redshift-space distortions, Phys. Rev. D 99, 083512 (2019).

[52] F. Simpson et al., CFHTLenS: Testing the laws of gravity with tomographic weak lensing and redshift-space distortions, Mon. Not. R. Astron. Soc. 429, 2249 (2013).

[53] P. A. R. Ade et al. (Planck Collaboration), Planck 2015 results. XIV. Dark energy and modified gravity, Astron. Astrophys. 594, A14 (2016).

[54] S. Joudaki et al., KiDS-450: testing extensions to the standard cosmological model, Mon. Not. R. Astron. Soc. 471, 1259 (2017).

[55] C. Garcia-Quintero and M. Ishak, Singling out modified gravity parameters and datasets reveals a new dichotomy between Planck and lensing, arXiv:2009.01189.

[56] E. V. Linder, Limited modified gravity, J. Cosmol. Astropart. Phys. 10 (2020) 042.

[57] N. Aghanim et al. (Planck Collaboration), Planck 2018 results. VI. Cosmological parameters, Astron. Astrophys. 641, A6 (2020).

[58] M. Ata et al., The clustering of the SDSS-IV extended Baryon Oscillation Spectroscopic Survey DR14 quasar sample: first measurement of baryon acoustic oscillations between redshift 0.8 and 2.2, Mon. Not. R. Astron. Soc. 473, 4773 (2018).

[59] V. de Sainte Agathe et al., Baryon acoustic oscillations at $z=2.34$ from the correlations of Ly $\alpha$ absorption in eBOSS DR14, Astron. Astrophys. 629, A85 (2019).

[60] M. Blomqvist et al., Baryon acoustic oscillations from the cross-correlation of Ly $\alpha$ absorption and quasars in eBOSS DR14, Astron. Astrophys. 629, A86 (2019).

[61] S. Alam et al. (eBOSS Collaboration), The Completed SDSS-IV extended Baryon Oscillation Spectroscopic Survey: Cosmological Implications from two Decades of Spectroscopic Surveys at the Apache Point observatory, arXiv:2007.08991.

[62] E. Krause, T. Eifler et al. (DES Collaboration), Dark Energy Survey Year 1 Results: Multi-Probe Methodology and Simulated Likelihood Analyses, arXiv:1706.09359.
[63] R. E. Smith, J. A. Peacock, A. Jenkins, S. D. M. White, C. S. Frenk, F. R. Pearce, P. A. Thomas, G. Efstathiou, and H. M. P. Couchmann (Virgo Consortium Collaboration), Stable clustering, the halo model and non-linear cosmological power spectra, Mon. Not. R. Astron. Soc. 341, 1311 (2003).

[64] S. Bird, M. Viel, and M. G. Haehnelt, Massive neutrinos and the non-linear matter power spectrum, Mon. Not. R. Astron. Soc. 420, 2551 (2012).

[65] R. Takahashi, M. Sato, T. Nishimichi, A. Taruya, and M. Oguri, Revising the Halofit model for the nonlinear matter power spectrum, Astrophys. J. 761, 152 (2012).

[66] D. N. Limber, The analysis of counts of the extragalactic nebulae in terms of a fluctuating density field, Astrophys. J. 117, 134 (1953).

[67] M. LoVerde and N. Afshordi, Extended Limber approximation, Phys. Rev. D 78, 123506 (2008).

[68] S. Bridle and L. King, Dark energy constraints from cosmic shear power spectra: Impact of intrinsic alignments on photometric redshift requirements, New J. Phys. 9, 444 (2007).

[69] www.cosmostat.org/software/nicaea.

[70] M. Kilbinger et al., Dark-energy constraints and correlations with systematics from CFHTLS weak lensing, SNLS supernovae Ia and WMAP5, Astron. Astrophys. 497, 677 (2009).

[71] P. A. R. Ade et al. (Planck Collaboration), Planck 2015 results XIII. Cosmological parameters, Astron. Astrophys. 594, A13 (2016).

[72] D. Brout et al. (DES Collaboration), First cosmology results using type ia supernovae from the dark energy survey: analysis, systematic uncertainties, and validation, Astrophys. J. 874, 150 (2019).

[73] D. M. Scolnic et al., The complete light-curve sample of spectroscopically confirmed sne ia from pan-starrs 1 and cosmological constraints from the combined pantheon sample, Astrophys. J. 859, 101 (2018).

[74] J. Marriner, J. Bernstein, R. Kessler, H. Lampeitl, R. Miquel, J. Mosher, R. C. Nichol, M. Sako, and M. Smith (SDSS Collaboration), A more general model for the intrinsic scatter in type Ia supernova distance moduli, Astrophys. J. 740, 72 (2011).

[75] R. Kessler and D. Scolnic, Correcting type Ia supernova distances for selection biases and contamination in photometrically identified samples, Astrophys. J. 836, 56 (2017).

[76] G. Efstathiou and J. R. Bond, Cosmic confusion: Degeneracies among cosmological parameters derived from measurements of microwave background anisotropies, Mon. Not. R. Astron. Soc. 304, 75 (1999).

[77] http://camb.info.

[78] A. Lewis, A. Challinor, and A. Lasenby, Efficient computation of CMB anisotropies in closed FRW models, Astrophys. J. 538, 473 (2000).

[79] C. Howlett, A. Lewis, A. Hall, and A. Challinor, CMB power spectrum parameter degeneracies in the era of precision cosmology, J. Cosmol. Astropart. Phys. 04 (2012) 027.

[80] J. A. Frieman, D. Huterer, E. V. Linder, and M. S. Turner, Probing dark energy with supernovae: Exploiting 
complementarity with the cosmic microwave background, Phys. Rev. D 67, 083505 (2003).

[81] A. Drlica-Wagner et al. (DES Collaboration), Dark energy survey year 1 results: The photometric data set for cosmology, Astrophys. J. Suppl. Ser. 235, 33 (2018).

[82] B. Flaugher, H. T. Diehl et al. (DES Collaboration), The dark energy camera, Astron. J. 150, 150 (2015).

[83] S. Desai et al. (BCS Collaboration), The blanco cosmology survey: Data acquisition, processing, calibration, quality diagnostics and data release, Astrophys. J. 757, 83 (2012).

[84] I. Sevilla et al. (DES Collaboration), in Particles and fields. Proceedings, Meeting of the Division of the American Physical Society, DPF 2011, Providence, USA (2011).

[85] J. J. Mohr et al. (DES Collaboration), The dark energy survey data management system, Proc. SPIE Int. Soc. Opt. Eng. 7016, 70160L (2008).

[86] E. Morganson et al. (DES Collaboration), The dark energy survey image processing pipeline, Publ. Astron. Soc. Pac. 130, 074501 (2018).

[87] E. Rozo et al. (DES Collaboration), redMaGiC: Selecting luminous red galaxies from the DES Science Verification data, Mon. Not. R. Astron. Soc. 461, 1431 (2016).

[88] E. Huff and R. Mandelbaum, Metacalibration: Direct Self-Calibration of Biases in Shear Measurement, arXiv: 1702.02600.

[89] E. S. Sheldon and E. M. Huff, Practical weak lensing shear measurement with metacalibration, Astrophys. J. 841, 24 (2017).

[90] .https://github.com/esheldon/ngmix

[91] D. Coe, N. Benitez, S. F. Sanchez, M. Jee, R. Bouwens, and H. Ford, Galaxies in the hubble ultra deep field. 1. Detection, multiband photometry, photometric redshifts, and morphology, Astron. J. 132, 926 (2006).

[92] J. Zuntz, E. Sheldon et al. (DES Collaboration), Dark Energy Survey Year 1 results: Weak lensing shape catalogues, Mon. Not. R. Astron. Soc. 481, 1149 (2018).

[93] B. Hoyle, D. Gruen et al. (DES Collaboration), Dark Energy Survey Year 1 Results: Redshift distributions of the weak-lensing source galaxies, Mon. Not. R. Astron. Soc. 478, 592 (2018).

[94] J. Elvin-Poole et al. (DES Collaboration), Dark energy survey year 1 results: Galaxy clustering for combined probes, Phys. Rev. D 98, 042006 (2018).

[95] J. Prat, C. Sanchez et al. (DES Collaboration), Dark Energy Survey year 1 results: Galaxy-galaxy lensing, Phys. Rev. D 98, 042005 (2018).

[96] M. A. Troxel et al. (DES Collaboration), Dark Energy Survey Year 1 results: Cosmological constraints from cosmic shear, Phys. Rev. D 98, 043528 (2018).

[97] M. Jarvis, G. Bernstein, and B. Jain, The skewness of the aperture mass statistic, Mon. Not. R. Astron. Soc. 352, 338 (2004).

[98] E. Krause and T. Eifler, COSMOLIKE-cosmological likelihood analyses for photometric galaxy surveys, Mon. Not. R. Astron. Soc. 470, 2100 (2017).

[99] A. Cooray and R. K. Sheth, Halo models of large scale structure, Phys. Rep. 372, 1 (2002).

[100] M. A. Troxel et al. (DES Collaboration), Survey geometry and the internal consistency of recent cosmic shear measurements, Mon. Not. R. Astron. Soc. 479, 4998 (2018).

[101] M. Crocce et al. (DES Collaboration), Dark energy survey year 1 results: Galaxy sample for BAO Measurement, Mon. Not. R. Astron. Soc. 482, 2807 (2019).

[102] S. Avila et al. (DES Collaboration), Dark energy survey year 1 results: Galaxy mock catalogues for BAO, Mon. Not. R. Astron. Soc. 479, 94 (2018).

[103] A. J. Ross et al., Optimized clustering estimators for bao measurements accounting for significant redshift uncertainty, Mon. Not. R. Astron. Soc. 472, 4456 (2017).

[104] H. Camacho et al. (DES Collaboration), Dark energy survey year 1 results: Measurement of the galaxy angular power spectrum, Mon. Not. R. Astron. Soc. 487, 3870 (2019).

[105] K. C. Chan et al. (DES Collaboration), BAO from angular clustering: Optimization and mitigation of theoretical systematics, Mon. Not. R. Astron. Soc. 480, 3031 (2018).

[106] D. A. Goldstein et al. (DES Collaboration), Automated transient identification in the dark energy survey, Astron. J. 150, 82 (2015); , 150, 165(E) (2015).

[107] R. Kessler et al. (DES Collaboration), THE difference imaging pipeline for the transient search in the dark energy survey, Astron. J. 150, 172 (2015).

[108] D. L. Burke et al. (DES Collaboration), Forward global photometric calibration of the dark energy survey, Astron. J. 155, 41 (2017).

[109] J. Lasker et al. (DES Collaboration), First cosmology results using type Ia supernovae from the dark energy survey: effects of chromatic corrections to supernova photometry on measurements of cosmological parameters, Mon. Not. R. Astron. Soc. 485, 5329 (2019).

[110] D. Brout et al. (DES Collaboration), First cosmology results using Type ia supernovae from the dark energy survey: Photometric pipeline and light-curve data release, Astrophys. J. 874, 106 (2019).

[111] M. Smith, C. B. D'Andrea et al. (DES Collaboration), First cosmology results using supernovae ia from the dark energy survey: survey overview, performance, and supernova spectroscopy, Astron. J. 160, 267 (2020).

[112] R. Kessler et al. (DES Collaboration), First cosmology results using type Ia supernova from the dark energy survey: Simulations to correct supernova distance biases, Mon. Not. R. Astron. Soc. 485, 1171 (2019).

[113] D. Scolnic and R. Kessler, Measuring type ia supernova populations of stretch and color and predicting distance biases, Astrophys. J. 822, L35 (2016).

[114] M. Hicken, W. M. Wood-Vasey, S. Blondin, P. Challis, S. Jha, P. L. Kelly, A. Rest, and R. P. Kirshner, Improved dark energy constraints from 100 new CfA supernova Type Ia light curves, Astrophys. J. 700, 1097 (2009).

[115] M. Hicken et al., , Astrophys. J. Suppl. Ser. 200, 12 (2012).

[116] C. Contreras et al., The carnegie supernova project: First photometry data release of low-redshift Type Ia supernovae, Astron. J. 139, 519 (2010).

[117] .https://www.sdss3.org/science/boss_publications.php

[118] F.-S. Kitaura et al., The clustering of galaxies in the SDSSIII Baryon Oscillation Spectroscopic Survey: mock galaxy catalogues for the BOSS Final Data Release, Mon. Not. R. Astron. Soc. 456, 4156 (2016). 
[119] A. G. Sanchez et al. (BOSS Collaboration), The clustering of galaxies in the completed SDSS-III Baryon Oscillation Spectroscopic Survey: combining correlated Gaussian posterior distributions, Mon. Not. R. Astron. Soc. 464, 1493 (2017).

[120] https://bitbucket.org/joezuntz/cosmosis/

[121] J. Zuntz, M. Paterno, E. Jennings, D. Rudd, A. Manzotti, S. Dodelson, S. Bridle, S. Sehrish, and J. Kowalkowski, CosmoSIS: Modular cosmological parameter estimation, Astron. Comput. 12, 45 (2015).

[122] F. Feroz and M. P. Hobson, Multimodal nested sampling: An efficient and robust alternative to Markov Chain Monte Carlo methods for astronomical data analyses, Mon. Not. R. Astron. Soc. 384, 449 (2008).

[123] F. Feroz, M. P. Hobson, and M. Bridges, , Mon. Not. R. Astron. Soc. 398, 1601 (2009).

[124] F. Feroz, M. Hobson, E. Cameron, and A. Pettitt, MultiNest: An efficient and robust Bayesian inference tool for cosmology and particle physics, Open J. Astrophys. 2, 10 (2019).

[125] W. J. Handley, M. P. Hobson, and A. N. Lasenby, POLYCHORD: Nested sampling for cosmology, Mon. Not. R. Astron. Soc. 450, L61 (2015).

[126] A. Lewis, GetDist: MCMC sample analysis, plotting and GUI (2019).

[127] P. Lemos, F. Köhlinger, W. Handley, B. Joachimi, L. Whiteway, and O. Lahav, Quantifying Suspiciousness within correlated data sets, Mon. Not. R. Astron. Soc. 496, 4647 (2020).

[128] .https://github.com/williamjameshandley/anesthetic

[129] W. Handley, Anesthetic: Nested sampling visualisation, J. Open Source Software 4, 1414 (2019).

[130] M. Raveri and W. Hu, Concordance and discordance in cosmology, Phys. Rev. D 99, 043506 (2019).

[131] W. Handley and P. Lemos, Quantifying dimensionality: Bayesian cosmological model complexities, Phys. Rev. D 100, 023512 (2019).

[132] F. Beutler et al. (BOSS Collaboration), The clustering of galaxies in the SDSS-III Baryon Oscillation Spectroscopic Survey: signs of neutrino mass in current cosmological data sets, Mon. Not. R. Astron. Soc. 444, 3501 (2014).

[133] I. G. Mccarthy, S. Bird, J. Schaye, J. Harnois-Deraps, A. S. Font, and L. Van Waerbeke, The BAHAMAS project: the CMB-large-scale structure tension and the roles of massive neutrinos and galaxy formation, Mon. Not. R. Astron. Soc. 476, 2999 (2018).

[134] V. Poulin, K. K. Boddy, S. Bird, and M. Kamionkowski, Implications of an extended dark energy cosmology with massive neutrinos for cosmological tensions, Phys. Rev. D 97, 123504 (2018).

[135] Y. Dirian, Changing the Bayesian prior: Absolute neutrino mass constraints in nonlocal gravity, Phys. Rev. D 96, 083513 (2017).

[136] S. Samuroff et al. (DES Collaboration), Dark Energy Survey Year 1 results: constraints on intrinsic alignments and their colour dependence from galaxy clustering and weak lensing, Mon. Not. R. Astron. Soc. 489, 5453 (2019).
[137] Dark Energy Survey (DES) and SPT Collaborations, Dark energy survey year 1 results: joint analysis of galaxy clustering, galaxy lensing, and $\mathrm{cmb}$ lensing two-point functions, Phys. Rev. D 100, 023541 (2019).

[138] K. Aylor, M. Joy, L. Knox, M. Millea, S. Raghunathan, and W. K. Wu, Sounds discordant: Classical distance ladder \& $\Lambda$ CDM-based determinations of the cosmological sound horizon, Astrophys. J. 874, 4 (2019).

[139] J. L. Bernal, L. Verde, and A. G. Riess, The trouble with $H_{0}$, J. Cosmol. Astropart. Phys. 10 (2016) 019.

[140] K. L. Pandey, T. Karwal, and S. Das, Alleviating the $H_{0}$ and $\sigma_{8}$ anomalies with a decaying dark matter model, J. Cosmol. Astropart. Phys. 07 (2020) 026.

[141] T. Tröster et al., Cosmology from large-scale structure, Astron. Astrophys. 633, L10 (2020).

[142] G. D’Amico, J. Gleyzes, N. Kokron, K. Markovic, L. Senatore, P. Zhang, F. Beutler, and H. Gil-Marín, The cosmological analysis of the SDSS/BOSS data from the effective field theory of large-scale structure, J. Cosmol. Astropart. Phys. 05 (2020) 005.

[143] M. M. Ivanov, M. Simonović, and M. Zaldarriaga, Cosmological parameters and neutrino masses from the final Planck and full-shape BOSS data, Phys. Rev. D 101, 083504 (2020).

[144] C.-H. To et al. (Dark Energy Survey Collaboration), Combination of cluster number counts and two-point correlations: Validation on Mock Dark Energy Survey, arXiv:2008.10757.

[145] E. J. Baxter et al. (DES Collaboration), Dark Energy Survey Year 1 results: Methodology and projections for joint analysis of galaxy clustering, galaxy lensing, and CMB lensing two-point functions, Phys. Rev. D 99, 023508 (2019).

[146] E. Jones, T. Oliphant, P. Peterson et al., SciPy: Open source scientific tools for PYTHON (2001).

[147] T. E. Oliphant, A guide to NumPy (Trelgol Publishing, USA, 2006).

[148] J. D. Hunter, Matplotlib: A 2D graphics environment, Comput. Sci. Eng. 9, 90 (2007).

[149] J. Schaye, C. Dalla Vecchia, C. M. Booth, R. P. C. Wiersma, T. Theuns, M. R. Haas, S. Bertone, A. R. Duffy, I. G. McCarthy, and F. van de Voort, The physics driving the cosmic star formation history, Mon. Not. R. Astron. Soc. 402, 1536 (2010).

[150] J. A. Blazek, N. MacCrann, M. A. Troxel, and X. Fang, Beyond linear galaxy alignments, Phys. Rev. D 100, 103506 (2019).

[151] N. Padmanabhan et al. (SDSS Collaboration), The clustering of luminous red galaxies in the Sloan Digital Sky Survey imaging data, Mon. Not. R. Astron. Soc. 378, 852 (2007).

[152] G. M. Bernstein, Comprehensive two-point analyses of weak gravitational lensing surveys, Astrophys. J. 695, 652 (2009).

[153] P. McDonald and A. Roy, Clustering of dark matter tracers: Generalizing bias for the coming era of precision LSS, J. Cosmol. Astropart. Phys. 08 (2009) 020. 
[154] T. Baldauf, U. Seljak, V. Desjacques, and P. McDonald, Evidence for quadratic tidal tensor bias from the halo bispectrum, Phys. Rev. D 86, 083540 (2012).

[155] J. E. McEwen, X. Fang, C. M. Hirata, and J. A. Blazek, FAST-PT: A novel algorithm to calculate convolution integrals in cosmological perturbation theory, J. Cosmol. Astropart. Phys. 09 (2016) 015.

[156] J. DeRose et al. (DES Collaboration), The Buzzard Flock: Dark Energy Survey Synthetic Sky Catalogs, arXiv:1901 .02401 . 